SLAC-PUB-7088

CERN-TH/96-56

March 1996

\title{
INDIRECT PROBES OF NEW PHYSICS
}

\author{
J.L. Hewett ${ }^{a}$, T. Takeuchi ${ }^{b}$, S. Thomas ${ }^{a}$ \\ ${ }^{a}$ Stanford Linear Accelerator Center, Stanford University, Stanford, CA 94309 \\ ${ }^{b}$ CERN, TH-Division, CH-1211 Geneva 23, Switzerland
}

Contributors: G. Bélanger, C. Burgess, D. Caldwell, U. Chattopadhyay, R.S. Chivukula, T. Goto, Y. Grossman, D. Kennedy, R.N. Mohapatra, P. Nath, T. Nihei, Y. Nir, Y. Okada, T.G. Rizzo, D. Silverman, E.H. Simmons, J. Terning, J.D. Wells

\begin{abstract}
We summarize the indirect effects of new physics in a variety of processes. We consider precision electroweak measurements, the $g-2$ of the muon, rare decays, meson mixing, $\mathrm{CP}$ violation, lepton number violating interactions, double beta decay, and the electric dipole moments of atoms, molecules, and the neutron. We include discussions of both model independent and dependent analyses where applicable.
\end{abstract}

To appear as a chapter in Electroweak Symmetry Breaking and Beyond the Standard Model, edited by T. Barklow, S. Dawson, H.E. Haber, and S. Siegrist, World Scientific.

\footnotetext{
${ }^{1}$ Work supported by the Department of Energy, Contract DE-AC03-76SF00515
} 


\section{Table of Contents}

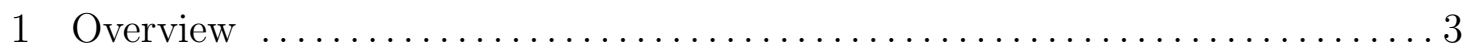

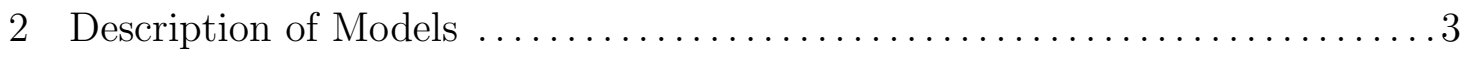

3 Precision Electroweak Measurements $\ldots \ldots \ldots \ldots \ldots \ldots \ldots \ldots \ldots \ldots \ldots$

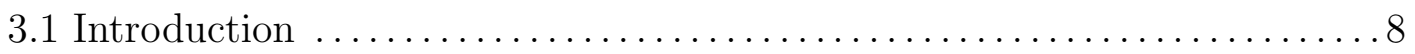

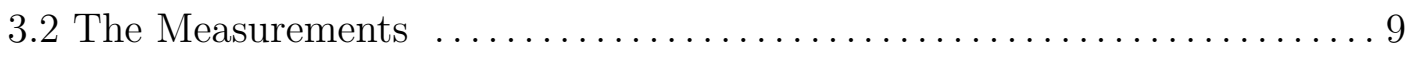

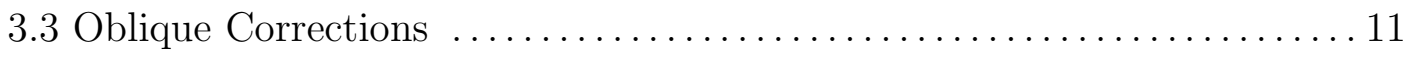

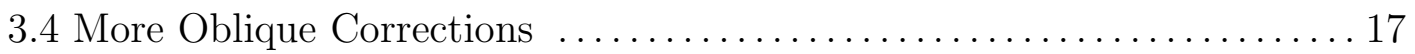

3.5 Non-Oblique Corrections $\ldots \ldots \ldots \ldots \ldots \ldots \ldots \ldots \ldots \ldots \ldots \ldots \ldots \ldots \ldots \ldots \ldots \ldots \ldots \ldots \ldots$

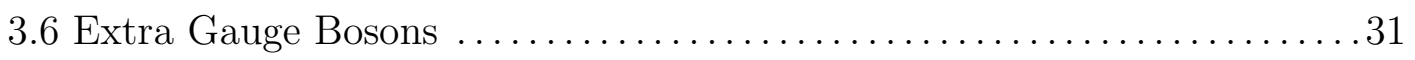

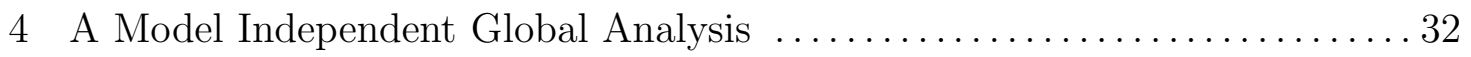

4.1 The Lowest-Dimension Effective Interactions $\ldots \ldots \ldots \ldots \ldots \ldots \ldots \ldots \ldots$

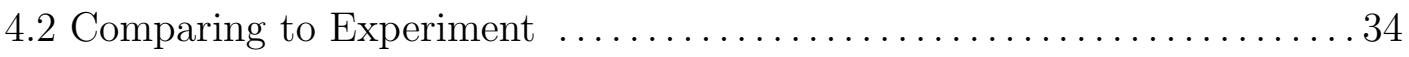

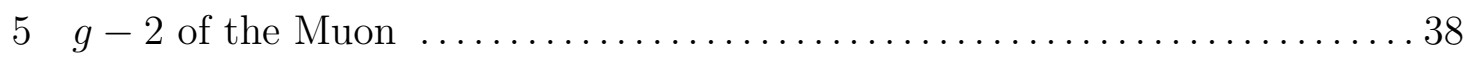

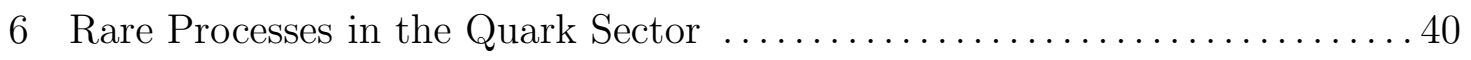

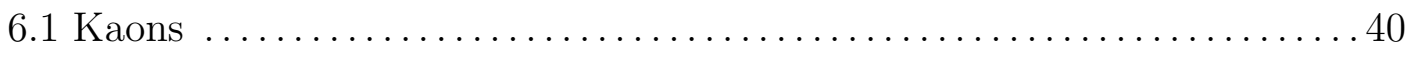

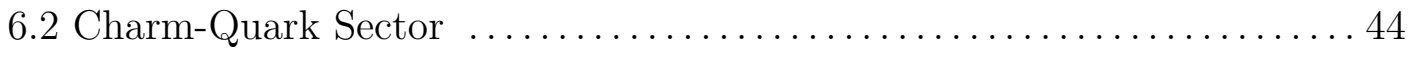

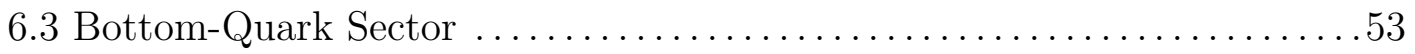

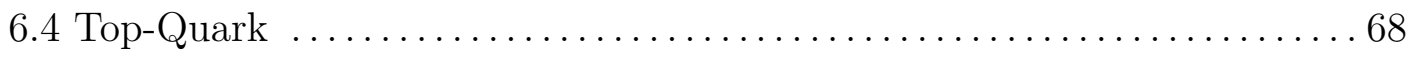

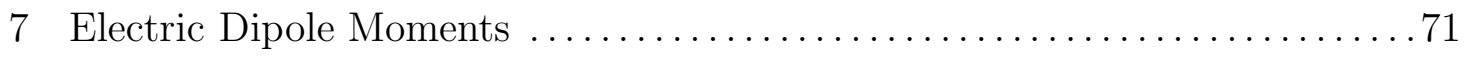

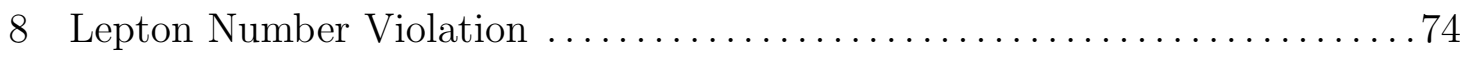

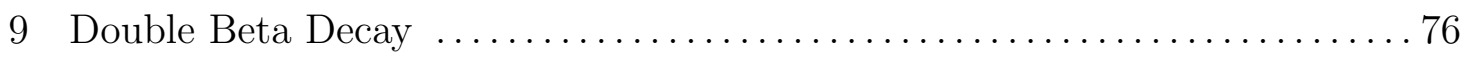

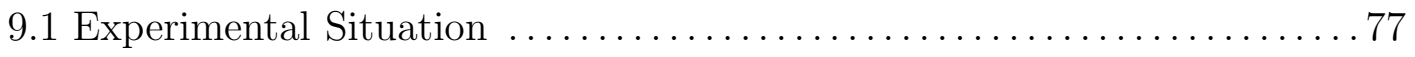

9.2 Neutrinoless Double Beta Decay and Physics Beyond the Standard Model 78

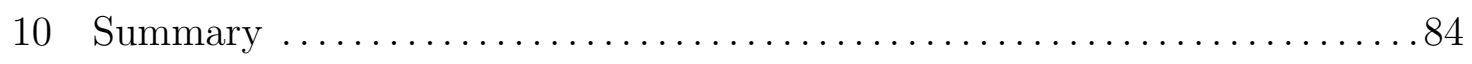




\section{Overview}

The investigation of virtual effects of new physics provides an important opportunity to probe the presence of interactions beyond the Standard Model (SM). Various types of experiments may expose the existence of new physics, including the search for direct production of new particles at high energy accelerators. Although this scenario has the advantage in that it would yield the cleanest observation of new physics, it is limited by the kinematic reach and accumulated luminosity of colliders. A complementary approach is offered by examining the indirect effects of new interactions in higher order processes and testing for deviations from SM predictions. In this case, one probes (i) the radiative corrections to perturbatively calculable processes, as well as (ii) transitions which are either suppressed or forbidden in the SM. Both of these scenarios carry the advantage of being able to explore the existence of new physics at very high energy scales. In fact, studies of new loop induced couplings can provide a means of probing the detailed structure of the SM at the level of radiative corrections where Glashow-Iliopoulos-Maiani (GIM) cancellations are important. As will be demonstrated below, in some cases the constraints on new degrees of freedom via indirect effects surpass those obtainable from collider searches. In other cases, entire classes of models are found to be incompatible with the data. Given the large amount of high luminosity 'lowenergy' data which is presently available and will continue to accumulate during the next decade, the loop effects of new interactions in rare processes and precision measurements will play a major role in the search for physics beyond the SM.

In this report we will simultaneously follow both model independent and dependent approaches, wherever possible, in determining the effects of new physics. In the following section, we first describe the general features of the various models which we consider throughout the chapter. We then examine the capacity of precision electroweak measurements to probe new interactions, paying special attention

to the current discrepancy between measurements and the SM prediction for the $Z b \bar{b}$ (and $Z c \bar{c}$ ) vertex, as well considering oblique corrections. Next we study the classic example of precision tests, the $g-2$ of the muon. We then turn our attention to SM suppressed or forbidden processes, such as rare decays, meson mixing, and $\mathrm{CP}$ violation, in the Kaon, charm-, bottom-, and top-quark systems. The effects of new physics on the electric dipole moments of atoms, molecules, and the neutron are then investigated. Finally, we focus on probes of the leptonic sector by examining lepton number violating processes, including double beta decay.

\section{Description of Models}

In this section we briefly summarize the general classes and main characteristics of models containing new physics that will be discussed in this report. 


\section{- Additional Fermions}

New fermions are predicted to exist in many extensions of the SM. In most models they generally carry the usual baryon and lepton number assignments, but can have unconventional electroweak quantum numbers. They can be classified according to their $\mathrm{SU}(2)_{L} \times \mathrm{U}(1)_{Y}$ assignments in the following manner [1]: $(i)$ Sequential fermions. The possibility of a fourth family of fermions has long been a popular extension to the SM. LEP/SLC data restricts the fourth neutrino to be heavy, i.e., $m_{\nu_{4}} \gtrsim M_{Z} / 2$, and the mixing between $\nu_{4}$ and $\nu_{e, \mu}$ to be small[2]. The recent LEP 1.5 run in November 1995 places the preliminary constraint 3 $m_{\nu_{4}}>48.1-60.2 \mathrm{GeV}$, depending on whether the neutrino species is $e$ or $\mu$ and is Dirac or Majorana in nature. It is worth noting that such a heavy fourth neutrino could mediate a see-saw type mechanism [4] thus generating a small mass for $\nu_{e, \mu, \tau}$. Constraints on the masses of the charged fourth generation fermions are [5]: $m_{L_{4}}>45.1-46.4 \mathrm{GeV}$ from LEP I (again, the LEP 1.5 run places constraints [3 up to $60 \mathrm{GeV}$ on $L^{ \pm}$, with the exact limit depending on the mass of the associated neutral heavy lepton), $m_{b^{\prime}}>85 \mathrm{GeV}$ from CDF assuming that it decays via charged current interactions, and $m_{t^{\prime}} \gtrsim M_{Z} / 2$ from LEP/SLC. In principle one can search for a $t^{\prime}$-quark in the same manner as the top-search analyses at the Tevatron, however, the results of such a search are not yet reported. (ii) Vector Fermions. Numerous extensions of the SM contain fermions whose left- and right-handed components transform identically under $\mathrm{SU}(2)_{L}$. For example, $E_{6}$ grand unified theories contain 6 a vector-like color singlet weak iso-doublet as well as a vector, weak iso-singlet, $Q+-1 / 3$ color triplet. Global analyses of the bounds placed on these exotic fermions from flavor changing neutral currents (FCNC) are performed in Ref. [7]. (iii) Mirror Fermions. The chiral properties of mirror fermions are opposite to those of the ordinary fermions. They appear 8] in some theories which restore left-right symmetry at the electroweak symmetry breaking scale, as well as in some grand unified and lattice gauge theories. Global constraints on their properties from precision electroweak measurements can be found in Csaki and Csikor [8].

\section{- Extended Higgs Sector}

The possibility of an enlarged Higgs sector beyond the minimal one-doublet version of the SM is consistent with data and has received substantial attention in the literature [9]. In this report, we consider three such classes of models.

The most economical case is that of Two-Higgs-Doublet Models (2HDM), which contain 5 physical Higgs bosons, 2 scalars $h^{0}, H^{0}$, a pseudoscalar $A^{0}$, and 2 charged scalars $H^{ \pm}$. Two such models naturally avoid tree-level FCNC, and are denoted as Model I, where one doublet $\left(\phi_{2}\right)$ generates masses for all fermions and the second $\left(\phi_{1}\right)$ decouples from the fermion sector, and Model II, where $\phi_{2}$ gives mass to the up-type quarks, while the down-type quarks and charged leptons receive their mass from $\phi_{1}$. Each doublet receives a vacuum expectation value (vev) $v_{i}$, subject to 
the constraint that $v_{1}^{2}+v_{2}^{2}=v_{\mathrm{SM}}^{2}$. Here, we will mostly be concerned with the $H^{ \pm}$ interactions with the fermion sector, which are governed by the Lagrangian

$$
\begin{gathered}
\mathcal{L}=\frac{g}{2 \sqrt{2} M_{W}} H^{ \pm}\left[V_{i j} m_{u_{i}} A_{u} \bar{u}_{i}\left(1-\gamma_{5}\right) d_{j}+V_{i j} m_{d_{j}} A_{d} \bar{u}_{i}\left(1+\gamma_{5}\right) d_{j}\right. \\
\left.m_{\ell} A_{\ell} \bar{\nu}_{\ell}\left(1+\gamma_{5}\right) \ell\right]+ \text { h.c. }
\end{gathered}
$$

with $A_{u}=\cot \beta$ in both models and $A_{d}=A_{\ell}=-\cot \beta(\tan \beta)$ in Model I(II), where $\tan \beta \equiv v_{2} / v_{1}$. A review of the constraints placed on such models from a variety of rare processes can be found in Ref. [10].

Models with Three (or more) Higgs Doublets (3HDM) contain new CP violating phases, which can appear in charged scalar exchange. These models can also avoid tree-level FCNC by imposing discrete symmetries or by requiring that only one doublet couples to each quark sector. In the latter case, the interaction Lagrangian between the quark sector and the two physical charged Higgs bosons can be written as

$$
\mathcal{L}=\frac{g}{2 \sqrt{2} M_{W}} \sum_{i=1,2} H_{i}^{+} \bar{U}\left[Y_{i} M_{u} V_{C K M}\left(1-\gamma_{5}\right)+X_{i} M_{d} V_{C K M}\left(1+\gamma_{5}\right)\right] D=\text { h.c. }
$$

where $X$ and $Y$ are complex coupling constants that arise from the diagonalization of the charged scalar mixing matrix and obey the relation $\sum_{i=1,2} X_{i} Y_{i}^{*}=1$. A general phenomenological analysis of this model can be found in Ref. [1]

The Higgs sector may also be extended without natural flavor conservation. In these models the above requirement of a global symmetry which restricts each fermion type to receive mass from only one doublet is replaced [12 by approximate flavor symmetries which act on the fermion sector. The Yukawa couplings can then possess a structure which reflects the observed fermion mass and mixing hierarchy. This allows the low-energy FCNC bounds to be evaded as the flavor changing couplings to the light fermions are small. Here, we will employ the Cheng-Sher ansatz 12, where the flavor changing couplings of the neutral Higgs to two fermions of different flavor are $\lambda_{h^{0} f_{i} f_{j}}=\left(\sqrt{2} G_{F}\right)^{1 / 2} \sqrt{m_{i} m_{j}} \Delta_{i j}$, with the $m_{i(j)}$ being the relevant fermion masses and $\Delta_{i j}$ representing a combination of mixing angles. The exact form of $\Delta_{i j}$ is calculable within a specific model.

\section{- Supersymmetry}

Supersymmetry (SUSY) relates the properties of bosons and fermions and a result of this symmetry is that all particles have supersymmetric partners with the same mass and gauge interactions, but with spin differing by $1 / 2$. For the SM particle content this predicts the existence of squarks, sleptons, gauginos, gluinos, and higgsinos. These sparticles have not yet been experimentally detected, and hence supersymmetry must be broken. There are theoretical and experimental reasons 13 (associated with, for example, the stability of the scale hierarchy in grand unified theories (GUTS), and with the consistency of measurements of the 
gauge couplings with these GUTS) to believe that the SUSY is broken near the scale of $1 \mathrm{TeV}$. The minimal supersymmetric standard model (MSSM) is the simplest version of SUSY; it contains the minimal number of new particles with the Higgs spectrum of the 2HDM Model II discussed above, and leads to the conservation of a multiplicative quantum number denoted as R-parity. Ordinary particles have R-parity of +1 , while sparticles possess negative R-parity. Hence in MSSM, only pairs of sparticles can be produced or exchanged in loops. In SUSY GUTS the sparticle mass and mixing spectrum can be described by a smaller set of parameters which relate the physical particles at the GUT scale. Assuming unification at a high-energy scale, we can take these parameters to be the common soft-breaking gaugino mass $m_{1 / 2}$, the universal scalar mass $m_{0}$, the supersymmetric higgsino mass parameter $\mu$, the universal trilinear soft-breaking term in the superpotential $A$, as well as $\tan \beta$ defined above. A general analysis of SUSY can be found in Ref. [13.

In non-minimal SUSY models R-parity can be broken; this leads to a very different SUSY phenomenology as sparticles can now be singly produced or exchanged in loops. These models still contain the minimal superfield content, but break R-parity either spontaneously, by the sneutrino acquiring a vev, or explicitly through terms contained in the superpotential. In the latter case, these terms take the form

$$
W=\lambda_{i j k} L_{i} L_{j} E_{k}^{c}+\lambda_{i j k}^{\prime} L_{i} Q_{j} D_{k}^{c}+\lambda_{i j k}^{\prime \prime} U_{i}^{c} D_{j}^{c} D_{k}^{c}
$$

where $i j k$ are generation indices, the $\lambda$ 's are a priori unknown Yukawa coupling constants, and $Q, L, U, D, E$ represent the chiral superfields. In order to preserve proton stability, the lepton and baryon number violating terms cannot simultaneously exist. Restrictions on the value of the Yukawa constants (the $\lambda$ 's) have been obtained 14 from a large variety of low-energy processes. The typical bounds are found to lie in the range

$$
\lambda_{i j k}^{\left(,^{\prime \prime}\right)} \leq(0.01-0.50) \frac{m_{\tilde{f}}}{100 \mathrm{GeV}}
$$

where $m_{\tilde{f}}$ represents the appropriate sparticle mass.

\section{- GUTS Models}

There are many classes of models with extended gauge sectors. One of the most popular cases is that of the Left-Right Symmetric Model (LRM) 15 which is based on the enlarged gauge group $S U(2)_{L} \times S U(2)_{R} \times U(1)$. Such theories have been fashionable for many years, as both a possible generalization of the SM and in the context of grand unified theories such as $\mathrm{SO}(10)$ and $E_{6}$. One prediction of these models is the existence of a heavy, charged, right-handed gauge boson $W_{R}^{ \pm}$, which in principle mixes with the SM $W_{L}^{ \pm}$via a mixing angle $\phi$ to form mass

eigenstates $W_{1,2}^{ \pm}$. This mixing angle is constrained [16] by data in polarized $\mu$ decay (in the case of light right-handed neutrinos) and from universality requirements to 
be $|\phi| \lesssim 0.05$. As we will see below, the virtual exchange of a $W_{R}^{ \pm}$can be felt in a variety of processes.

The Alternate Left-Right Symmetric Model (ALRM) [17] originates from $E_{6}$ GUTS and is also based on the low-energy gauge group $S U(2)_{L} \times S U(2)_{R} \times U(1)$. However, since a single generation in $E_{6}$ theories contains 27 2-component fermions (in contrast to the 16 fermions per generation in $\mathrm{SO}(10)$ ), quantum number ambiguities arise which allow the $T_{3 L(R)}$ assignments of the usual SM fermions to differ from those of the LRM for $\nu_{L, R}, e_{L}$, and $d_{R}$. This allows, for example, the righthanded $W$ boson to couple the $u_{R}^{i}$ to the exotic charged $-1 / 3$, vector singlet, color triplet fermion $h_{R}$, which is present in the 27 of $E_{6}$. This possibility can lead to some striking signatures 17].

\section{- Technicolor}

In technicolor theories the fundamental Higgs boson of the SM is replaced by fermion condensates which break the electroweak symmetry via vevs of the form $\langle 0|\Psi \bar{\Psi}| 0\rangle \alpha \Lambda_{T C}^{3}$. The new fermions $\Psi$ are known as technifermions and interact via a new technicolor force. The confinement scale of this new force is $\Lambda \sim 250$ $\mathrm{GeV}$. In order to generate masses for the SM fermions, additional extended technicolor (ETC) interactions, which couple the SM fermions to the technifermions, are usually introduced. This results in masses for the ordinary fermions of order $g_{E T C}^{2} \Lambda_{T C}^{3} / M_{E T C}^{2}$. For the typical value of $\Lambda_{T C}$ given above, it is clear that rather light ETC bosons, $M_{E T C} \sim \sqrt{\Lambda_{T C}^{3} / m_{f}} \lesssim \mathrm{TeV}$, are required to achieve adequate values for the fermion masses $m_{f}$. However, this TeV mass range for the ETC bosons leads to large contributions to FCNC and electroweak radiative corrections and hence potentially conflicts with experiment. These problems are not insurmountable, and more realistic technicolor models which address these issues are discussed in the sections below.

\section{- Leptoquarks}

Leptoquarks are color triplet particles which couple to a lepton-quark pair and are naturally present in many theories beyond the SM which relate leptons and quarks at a more fundamental level. They appear in Technicolor theories, models with quark-lepton substructure, horizontal symmetries, and grand unified theories based on the gauge groups $\mathrm{SU}(5), \mathrm{SO}(10)$, and $E_{6}$. In all these scenarios leptoquarks carry both baryon and lepton number, but their other quantum numbers, i.e., spin, weak isospin, and electric charge, can vary[1]. They couple to fermions via a Yukawa interaction with a priori unknown strength. This interaction is usually parameterized in terms of the fine structure constant as $\lambda_{L Q}^{2} / 4 \pi=F_{L Q} \alpha$. An investigation of the global constraints from $\mathrm{FCNC}$ on leptoquarks may be found in Ref. 18.

\section{- Anomalous Couplings}


Possible deviations from the SM form for the trilinear $W W \gamma$ and $W W Z$ vertex has received much attention[19]. These potentially anomalous vertices can be probed by looking for deviations from the SM in tree-level processes such as $e^{+} e^{-} \rightarrow W^{+} W^{-}$, or in loop order processes, for example the $g-2$ of the muon. In the latter case, cutoffs must generally be used in order to regulate the divergent loop integrals and can introduce errors by attributing a physical significance to the cutoff. We will see below that in some instances the GIM mechanism may be invoked to cancel such divergences yielding cut-off independent results. The CP-conserving interaction Lagrangian for $W W V$ interactions can be written as

$$
\begin{aligned}
\mathcal{L}_{W W V}=i g_{W W V}[ & \left(W_{\mu \nu}^{\dagger} W^{\mu} V^{\nu}-W_{\mu}^{\dagger} V_{\nu} W^{\mu \nu}\right)+\kappa_{V} W_{\mu}^{\dagger} W_{\nu} V^{\mu \nu}+\frac{\lambda_{V}}{M_{W}^{2}} W_{\lambda \mu}^{\dagger} W_{\nu}^{\mu} V^{\nu \lambda} \\
& \left.-i g_{5}^{V} \epsilon^{\mu \nu \lambda \rho}\left(W_{\mu}^{\dagger} \partial_{\lambda} W_{\nu}-W_{\nu} \partial_{\lambda} W_{\mu}^{\dagger}\right) V_{\rho}\right]
\end{aligned}
$$

where $V_{\mu \nu}=\partial_{\mu} V_{\nu}-\partial_{\nu} V_{\mu}, g_{W W V}=g c_{w}(e)$ for $V_{\mu}=Z_{\mu}\left(A_{\mu}\right)$, and the parameters $\left(\Delta \kappa_{V} \equiv \kappa_{V}-1\right)$ take on the values $\Delta \kappa_{V}, \lambda_{V}, g_{5}^{V}=0$ in the SM.

Anomalous couplings between the fermions and the gauge boson sector may also be probed in loop processes. In the case of the fermionic coupling to a neutral gauge boson, a general Lagrangian (assuming operators of dimension-five or less, only) can be written as

$$
\begin{aligned}
\mathcal{L}= & e \bar{f}_{i}\left[Q_{f} v_{\gamma} \gamma_{\mu}+\frac{i \sigma_{\mu \nu} q^{\nu}}{m_{f_{i}}+m_{f_{j}}}\left(\kappa_{\gamma}-i \tilde{\kappa}_{\gamma} \gamma_{5}\right)\right] f_{j} A^{\mu} \\
& +\frac{g}{2 c_{w}} \bar{f}_{i}\left[\gamma_{\mu}\left(v_{Z}-a_{Z} \gamma_{5}\right)+\frac{i \sigma_{\mu \nu} q^{\nu}}{m_{f_{i}}+m_{f_{j}}}\left(\kappa_{Z}-i \tilde{\kappa}_{Z} \gamma_{5}\right)\right] f_{j} Z^{\mu}
\end{aligned}
$$

where $Q_{f}$ represents the fermion's electric charge, $v_{\gamma, Z}, a_{Z}$ represent the fermion's vector and axial-vector coupling (where gauge invariance dictates that the photon be off-shell in the case of $i \neq j), \kappa(\tilde{\kappa})_{\gamma, Z}$ represent the anomalous magnetic (electric) dipole moment, and $q^{\nu}$ corresponds to the momentum of the gauge boson. We will discuss the bounds placed on these anomalous couplings below.

\section{Precision Electroweak Measurements}

\subsection{Introduction}

Virtual effects from new physics beyond the SM can manifest themselves in a number of ways: They can contribute to rare processes that are forbidden or highly suppressed within the SM (as discussed extensively in a separate section), or they can affect well measured and perturbatively calculable electroweak observables through radiative corrections and lead to detectable deviations between the SM predictions

and the experimentally measured values. The presence of such deviations, or the 
absence of an expected one, can give us important, albeit indirect, information on the nature and particle content of the yet to be discovered sectors beyond the SM.

This section is organized as follows: In the next subsection, we first review the current status of precision electroweak measurements. We then apply the methods developed in Refs. [20, 21, 22] to the data and express the possible size of oblique and non-oblique corrections from new physics in terms of limits to a few relatively model independent parameters.

\subsection{The Measurements}

The precision measurements of electroweak observables have heretofore been instrumental in verifying the validity of the electroweak sector of the SM, namely the $S U(2)_{L} \times U(1)_{Y}$ gauge theory of electroweak interactions [23. Today, with the SM firmly established and the experimental errors improving incrementally each year, they are our best hopes of seeing the SM fail at some level of precision and thereby establish the existence of new physics effects. In fact, such an effect may have already been seen at LEP/SLC where the ratios $R_{b} \equiv \Gamma_{b \bar{b}} / \Gamma_{\text {had }}$ and $R_{c} \equiv \Gamma_{c \bar{c}} / \Gamma_{\text {had }}$ have been measured to deviate from their SM predictions by $3.8 \sigma$ and $2.4 \sigma$, respectively.

In Table 1, we list the most recent precision measurements from the $e^{+} e^{-}$and $p \bar{p}$ collider experiments [24, 25], $\nu_{\mu}$ and $\bar{\nu}_{\mu}$ deep inelastic scattering experiments 26. 27], $\nu_{\mu} e$ and $\bar{\nu}_{\mu} e$ elastic scattering experiments [27, 28], and atomic parity violation (APV) experiments [29], together with the predictions of the observables in the SM with $m_{t}=180 \mathrm{GeV}$ [30] and $m_{H}=300 \mathrm{GeV}$. The accuracy of each measurement is shown in percentages and the disagreement between the theoretical and experimental central values are shown in units of the experimental error.

The SM predictions for the $W$ mass and the LEP/SLC observables were obtained using the program ZFITTER 4.9 [31], and the predictions for the low energy $\nu_{\mu} N, \nu_{\mu} e$, and APV observables were calculated from the formulae given in Ref. [32]. The values of the effective QED coupling constant and the $\overline{\mathrm{MS}} \mathrm{QCD}$ coupling constant at the $Z$ mass scale were chosen to be $\alpha^{-1}\left(M_{Z}\right)=128.9 \pm 0.1$ [33], and $\alpha_{s}\left(M_{Z}\right)=0.123 \pm 0.006$ [34] respectively. The errors on the SM predictions of $\Gamma_{Z}, \sigma_{\mathrm{h}}^{0}$, and $R_{\ell}$ are from the uncertainty on the value of $\alpha_{s}\left(M_{Z}\right)$. Additional errors due to the uncertainty in the values of $\alpha^{-1}\left(M_{Z}\right)$, quark masses, etc. are not shown.

Several comments are in order:

- The value of $\Gamma_{\ell^{+} \ell^{-}}$is derived from the values of the four line-shape parameters

$$
M_{Z}, \quad \Gamma_{Z}, \quad \sigma_{\mathrm{h}}^{0}=\frac{12 \pi}{M_{Z}^{2}} \frac{\Gamma_{\ell^{+} \ell^{-}} \Gamma_{\mathrm{had}}}{\Gamma_{Z}^{2}}, \quad R_{\ell}=\frac{\Gamma_{\mathrm{had}}}{\Gamma_{\ell^{+} \ell^{-}}},
$$

and their correlations. It is not an independent measurement, but the SM 
prediction of $\Gamma_{\ell^{+} \ell^{-}}$has the advantage of being free of the QCD uncertainties which plague the predictions for $\Gamma_{Z}, \sigma_{\mathrm{h}}^{0}$, and $R_{\ell}$.

- The forward backward asymmetries $A_{\mathrm{FB}}^{0, f}$ are defined as

$$
A_{\mathrm{FB}}^{0, f}=\frac{3}{4} A_{e} A_{f}
$$

with

$$
A_{f}=\frac{2 g_{V f} g_{A f}}{g_{V f}^{2}-g_{A F}^{2}}=\frac{g_{L f}^{2}-g_{R f}^{2}}{g_{L f}^{2}+g_{R f}^{2}},
$$

where $g_{V f}=g_{L f}+g_{R f}$ and $g_{A f}=g_{L f}-g_{R f}$ are the effective vector and axial-vector couplings of fermion $f$ to the $Z$. QCD corrections have been removed from $A_{\mathrm{FB}}^{0, b}$ and $A_{\mathrm{FB}}^{0, c}$.

- The effective weak angle $\sin ^{2} \theta_{\text {eff }}^{\text {lept }}$ is defined as

$$
\sin ^{2} \theta_{\mathrm{eff}}^{\mathrm{lept}} \equiv \frac{1}{4}\left(1-g_{V \ell} / g_{A \ell}\right)
$$

The LEP value of $\sin ^{2} \theta_{\text {eff }}^{\text {lept }}$ is the average of values extracted from the leptonic asymmetries $A_{\mathrm{FB}}^{0, \ell}, A_{e}$, and $A_{\tau}$ only. The SLC value of $\sin ^{2} \theta_{\mathrm{eff}}^{\text {lept }}$ is from $A_{L R}$.

- The parameters $g_{L}^{2}$ and $g_{R}^{2}$ measured in $\nu_{\mu}$ - and $\bar{\nu}_{\mu}$-nucleon scattering experiments [26] are defined as

$$
\begin{aligned}
& R_{\nu}=g_{L}^{2}+g_{R}^{2} r, \\
& R_{\bar{\nu}}=g_{L}^{2}+\frac{g_{R}^{2}}{r},
\end{aligned}
$$

where $R_{\nu}, R_{\bar{\nu}}$, and $r$ denote the following cross section ratios:

$$
\begin{aligned}
R_{\nu} & =\frac{\sigma\left(\nu_{\mu} N \rightarrow \nu_{\mu} X\right)}{\sigma\left(\nu_{\mu} N \rightarrow \mu^{-} X\right)} \\
R_{\bar{\nu}} & =\frac{\sigma\left(\bar{\nu}_{\mu} N \rightarrow \bar{\nu}_{\mu} X\right)}{\sigma\left(\bar{\nu}_{\mu} N \rightarrow \mu^{+} X\right)}, \\
r & =\frac{\sigma\left(\bar{\nu}_{\mu} N \rightarrow \mu^{+} X\right)}{\sigma\left(\nu_{\mu} N \rightarrow \mu^{-} X\right)} .
\end{aligned}
$$

It is customary for $\nu_{\mu} N$ scattering experiments to report a value of $s_{W}^{2} \equiv$ $1-M_{W}^{2} / M_{Z}^{2}$ which is obtained from $g_{L}^{2}$ and $g_{R}^{2}$ through the relations [35]

$$
\begin{aligned}
g_{L}^{2} & =\rho_{\nu N}^{2}\left(\frac{1}{2}-s_{\nu N}^{2}+\frac{5}{9} s_{\nu N}^{4}\right) \\
g_{R}^{2} & =\rho_{\nu N}^{2}\left(\frac{5}{9} s_{\nu N}^{4}\right)
\end{aligned}
$$


with

$$
s_{\nu N}^{2}=\kappa_{\nu N} s_{W}^{2},
$$

where $\rho_{\nu N}$ and $\kappa_{\nu N}$ represent radiative corrections [32]. $\left(\rho_{\nu N}=\kappa_{\nu N}=1\right.$ at tree level.) While this mode of presentation is convenient for comparing the result with the $p \bar{p}$ measurement of $M_{W}$ and checking the consistency of the $\mathrm{SM}$, the possible presence of new physics contributions in $\rho_{\nu N}$ and $\kappa_{\nu N}$ can potentially affect the extracted value of $s_{W}^{2}$. We have therefore converted the reported values of $s_{W}^{2}$ back into values of $g_{L}^{2}$ and $g_{R}^{2}$ since these are directly measured quantities and will be unaffected by the presence of new physics. The values quoted in Table 1 are the global averages from Ref. [27.

- Atomic parity violation (APV) experiments have already measured the amplitude of parity violating transitions in atoms to an accuracy of $1 \%$ to $2 \%$ for cesium [36], bismuth [37], lead [38], and thallium [39, 40]. Comparison of these APV results with the SM requires the extraction of the weak charge

$$
Q_{W}=\rho_{e q}^{\prime}\left[Z\left(1-4 s_{e q}^{\prime 2}\right)-N\right]
$$

which quantifies the coupling of the nucleus to the $Z$ boson [41]. However, this requires accurate atomic physics calculations which is currently available for only cesium and thallium [42]. The quoted value of $Q_{W}\left({ }_{55}^{133} \mathrm{Cs}\right)$ is from Ref. [36] using the result of Ref. [42], and that of $Q_{W}\left({ }_{81}^{205} \mathrm{Tl}\right)$ is the average of the value given in Ref. [39] and the value inferred by Rosner [29] from the result of Ref. [40].

A quick glance through Table 1 will give the reader a good idea on just how accurate modern precision measurements have become and how successful the SM is in predicting these results. Though the experimental error on many observables is a mere fraction of a percent, the only observables that deviate from the SM by

more than $1 \sigma$ are the LEP values of $R_{b}$ and $R_{c}$, the SLC values of $\sin ^{2} \theta_{\text {eff }}^{\text {lept }}$ and $A_{b}$, and $Q_{W}\left({ }_{55}^{133} \mathrm{Cs}\right)$ from $\mathrm{APV}$.

In the following, we will attempt to understand what these agreements and deviations imply about the types and sizes of possible radiative corrections from new physics.

\subsection{Oblique Corrections}

Before confronting the data given in Table 1 and trying to extract limits on the radiative corrections from new physics, it is worthwhile to discuss what type of corrections to expect.

We begin by noticing that all the precision electroweak measurements conducted so far involve interactions which at tree level are described by light fermions exchanging a single electroweak gauge boson $(\gamma, W$, or $Z)$. Radiative corrections 
Table 1: Determination of electroweak parameters as of summer 1995 [24]. The SM predictions are calculated for $m_{t}=180 \mathrm{GeV}, m_{H}=300 \mathrm{GeV}$ with $\alpha^{-1}\left(M_{Z}\right)=128.9$, $\alpha_{s}\left(M_{Z}\right)=0.123$.

\begin{tabular}{|c|c|c|c|c|}
\hline Observable & "Measurement & $\begin{array}{c}\text { Error } \\
(\%)\end{array}$ & SM prediction & $\begin{array}{r}\text { Deviation } \\
(\sigma)\end{array}$ \\
\hline \multicolumn{5}{|l|}{ LEP } \\
\hline \multicolumn{5}{|l|}{ line-shape: } \\
\hline$M_{Z}[\mathrm{GeV}]$ & $91.1884 \pm 0.0022$ & 0.0024 & used as input & \\
\hline$\Gamma_{Z}[\mathrm{GeV}]$ & $2.4963 \pm 0.0032$ & 0.13 & $2.4973 \pm 0.0032$ & -0.3 \\
\hline$\sigma_{\mathrm{h}}^{0}[\mathrm{nb}]$ & $41.488 \pm 0.078$ & 0.19 & $41.454 \pm 0.032$ & 0.4 \\
\hline$R_{\ell}$ & $20.788 \pm 0.032$ & 0.15 & $20.767 \pm 0.040$ & 0.7 \\
\hline$\Gamma_{\ell^{+} \ell^{-}}[\mathrm{MeV}]$ & $83.93 \pm 0.14$ & 0.17 & 83.98 & -0.4 \\
\hline \multicolumn{5}{|l|}{ lepton } \\
\hline $\begin{array}{l}\text { asymmetries: } \\
\sin ^{2} \theta_{\text {lept }}\end{array}$ & $0.23160 \pm 0.00049$ & 0.21 & 0.23179 & -0.4 \\
\hline silt & & & & \\
\hline \multicolumn{5}{|l|}{$\begin{array}{l}b \text { and } c \text { quark } \\
\text { results: }\end{array}$} \\
\hline$R_{b}$ & $0.2219 \pm 0.0017$ & 0.77 & 0.2155 & 3.8 \\
\hline$R_{c}$ & $0.1543 \pm 0.0074$ & 4.8 & 0.1724 & -2.4 \\
\hline$A_{\mathrm{FB}}^{0, b}$ & $0.0999 \pm 0.0031$ & 3.1 & 0.1016 & -0.5 \\
\hline$A_{\mathrm{FB}}^{0, c}$ & $0.0725 \pm 0.0058$ & 8.0 & 0.0725 & 0.0 \\
\hline \multicolumn{5}{|l|}{ SLC } \\
\hline $\sin ^{2} \theta_{\text {eff }}^{\text {lept }}$ & $0.23049 \pm 0.00050$ & 0.22 & 0.23179 & -2.6 \\
\hline$R_{b}$ & $0.2171 \pm 0.0054$ & 2.5 & 0.2155 & 0.3 \\
\hline$A_{b}$ & $0.841 \pm 0.053$ & 6.3 & 0.9345 & -1.8 \\
\hline$A_{c}$ & $0.606 \pm 0.090$ & 15 & 0.6671 & -0.7 \\
\hline \multicolumn{5}{|l|}{$\underline{p \bar{p} \text { colliders }}$} \\
\hline$\overline{M_{W}[\mathrm{GeV}]}$ & $80.26 \pm 0.16$ & 0.20 & 80.35 & -0.6 \\
\hline \multicolumn{5}{|l|}{$\nu N$ scattering } \\
\hline$\overline{g_{L}^{2}}$ & $0.3017 \pm 0.0033$ & 1.1 & 0.304 & -0.4 \\
\hline$g_{R}^{2}$ & $0.0326 \pm 0.0033$ & 10 & 0.030 & 0.8 \\
\hline \multicolumn{5}{|l|}{ 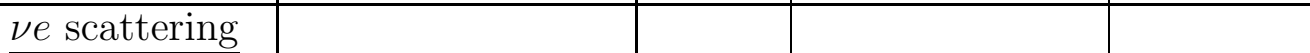 } \\
\hline$\overline{g_{A}^{\nu e}}$ & $-0.506 \pm 0.015$ & 3.0 & -0.507 & 0.07 \\
\hline$g_{V}^{\nu e}$ & $-0.039 \pm 0.017$ & 44 & -0.037 & -0.1 \\
\hline \multicolumn{5}{|l|}{ APV } \\
\hline$Q_{W}\left({ }_{55}^{133} \mathrm{Cs}\right)$ & $-71.0 \pm 1.8$ & 2.5 & -73.2 & 1.2 \\
\hline$Q_{W}\left({ }_{81}^{205} \mathrm{Tl}\right)$ & $-116.3 \pm 3.1$ & 2.7 & -116.8 & 0.2 \\
\hline
\end{tabular}


to such four-fermion processes come in three classes: vacuum polarization corrections, vertex corrections, and box corrections. The vacuum polarization corrections are often called 'oblique' corrections, as opposed to the 'direct' vertex and box corrections because they only affect the propagation and mixings of the gauge bosons and do not change the form of the interaction itself. They are independent of the external fermions and affect all processes that involve the exchange of the electroweak gauge boson universally. On the other hand, direct corrections do depend on the external fermions and are specific to each process.

For new physics to contribute to oblique corrections, they need only to carry $S U(2)_{L} \times U(1)_{Y}$ quantum numbers and they will contribute at the same order in $\alpha$ as the usual SM corrections. Furthermore, as we will see below, oblique corrections from new physics do not necessarily decouple when the new physics scale is taken to infinity.

However, for new physics to contribute to the direct vertex and box corrections at the 1-loop level, they must couple directly to the light external fermions. Such couplings can be expected to be highly suppressed: if the 'light' fermions coupled strongly to new and heavy physics, they would not be so light. (Recall that this is true for the Higgs sector of the SM. The Yukawa couplings of the Higgs boson to light fermions are suppressed by a factor of $m_{f}^{2} / M_{W}^{2}$ compared to the gauge couplings.) One exception is the $b$ quark: Because the $b$ quark is the isospin partner of the $t$ quark, the mechanism responsible for the generation of the large $t$ quark mass can also be expected to lead to a large correction to the $Z b \bar{b}$ vertex. Even within the SM the $Z b \bar{b}$ vertex receives an important correction from the $t-W$ loop.

Following these considerations, it seems reasonable to make the following three assumptions about radiative corrections from new physics.

1. The electroweak gauge group is the standard $S U(2)_{L} \times U(1)_{Y}$. The only electroweak gauge bosons are the photon, the $W^{ \pm}$, and the $Z$.

2. The couplings of new physics to light fermions are highly suppressed so that 'direct' corrections from new physics can be neglected (with the possible exception of processes involving the $b$ quark). Only oblique corrections need to be considered.

3. The new physics scale is large compared to the $W$ and $Z$ masses.

The first and second assumptions taken together means that we only need to consider new physics contributions to four vacuum polarization functions, namely, the self energies of the photon, $W$, and $Z$, and the $Z$-photon mixing. Using the notation

$$
\int d^{4} x e^{i q \cdot x}\left\langle J_{X}^{\mu}(x) J_{Y}^{\nu}(0)\right\rangle=i g^{\mu \nu} \Pi_{\mathrm{XY}}\left(q^{2}\right)+\left(q^{\mu} q^{\nu} \text { term }\right),
$$


where $J_{X}$ is the current that couples to gauge boson $X(X=\gamma, W, Z)$, we can write these functions as $\Pi_{\gamma \gamma}\left(q^{2}\right), \Pi_{\mathrm{WW}}\left(q^{2}\right), \Pi_{\mathrm{ZZ}}\left(q^{2}\right)$, and $\Pi_{\mathrm{Z} \gamma}\left(q^{2}\right)$. (Note that we do not need to consider the $q^{\mu} q^{\nu}$ parts of the vacuum polarization tensors because they only correct the longitudinal parts of the gauge boson propagators which are suppressed by a factor of $m_{f}^{2} / M_{W / Z}^{2}$ compared to the transverse parts when contracted with the external fermion currents.)

In general, the new physics contributions to $\Pi_{\gamma \gamma}\left(q^{2}\right), \Pi_{\mathrm{WW}}\left(q^{2}\right), \Pi_{\mathrm{ZZ}}\left(q^{2}\right)$, and $\Pi_{\mathrm{Z} \gamma}\left(q^{2}\right)$ are complicated functions of $q^{2}$. However, the third assumption allows us to expand the new physics contributions to these functions around $q^{2}=0$ in powers of $q^{2} / M_{\text {new }}^{2}$, where $M_{\text {new }}$ is the scale of new physics, and to keep only the first few terms. We will only keep the constant and linear terms in $q^{2}$ since higher order terms will decouple as $M_{\text {new }} \rightarrow \infty$ :

$$
\begin{aligned}
\Pi_{\gamma \gamma}\left(q^{2}\right) & =q^{2} \Pi_{\gamma \gamma}^{\prime}(0)+\cdots \\
\Pi_{\mathrm{Z} \gamma}\left(q^{2}\right) & =q^{2} \Pi_{\mathrm{Z} \gamma}^{\prime}(0)+\cdots \\
\Pi_{\mathrm{ZZ}}\left(q^{2}\right) & =\Pi_{\mathrm{ZZ}}(0)+q^{2} \Pi_{\mathrm{ZZ}}^{\prime}(0)+\cdots \\
\Pi_{\mathrm{WW}}\left(q^{2}\right) & =\Pi_{\mathrm{WW}}(0)+q^{2} \Pi_{\mathrm{WW}}^{\prime}(0)+\cdots
\end{aligned}
$$

It is to be understood that we are expanding only the part of the $\Pi_{\mathrm{XY}}\left(q^{2}\right)^{\text {'s }}$ that arise from new physics. This linear approximation permits us to express the new physics contributions in terms of just six parameters, namely $\Pi_{\gamma \gamma}^{\prime}(0), \Pi_{Z \gamma}^{\prime}(0)$, $\Pi_{\mathrm{ZZ}}(0), \Pi_{\mathrm{ZZ}}^{\prime}(0), \Pi_{\mathrm{WW}}(0)$, and $\Pi_{\mathrm{WW}}^{\prime}(0)$. Of these six, three will be absorbed into the renormalization of the three input parameters $\alpha, G_{\mu}$, and $M_{Z}$ rendering them unobservable. This leaves three parameters which are observable and finite and can be expressed as linear combinations of the original six. One popular choice for these linear combinations is given by [20]:

$$
\begin{aligned}
\alpha S & =4 s^{2} c^{2}\left[\Pi_{\mathrm{ZZ}}^{\prime}(0)-\frac{c^{2}-s^{2}}{s c} \Pi_{\mathrm{Z} \gamma}^{\prime}(0)-\Pi_{\gamma \gamma}^{\prime}(0)\right], \\
\alpha T & =\frac{\Pi_{\mathrm{WW}}(0)}{M_{W}^{2}}-\frac{\Pi_{\mathrm{ZZ}}(0)}{M_{Z}^{2}}, \\
\alpha U & =4 s^{2}\left[\Pi_{\mathrm{WW}}^{\prime}(0)-c^{2} \Pi_{\mathrm{ZZ}}^{\prime}(0)-2 s c \Pi_{\mathrm{Z} \gamma}^{\prime}(0)-s^{2} \Pi_{\gamma \gamma}^{\prime}(0)\right] .
\end{aligned}
$$

This definition enjoys the property that the parameters $T$ and $U$ will be zero when the new physics does not break custodial isospin symmetry. In fact, $\alpha T$ is just the shift of the $\rho$ parameter due to new physics:

$$
\rho=1+\delta \rho_{\mathrm{SM}}+\alpha T
$$

$S, T$, and $U$ can parameterize the oblique corrections from various extensions of the SM as long as they satisfy the three assumptions listed above. While this excludes models which extend the electroweak gauge group beyond the standard $S U(2)_{L} \times$ $U(1)_{Y}$ and which introduce new electroweak gauge bosons, they still encompass a 


\begin{tabular}{|c||c|c|c|}
\hline \hline & $S$ & $T$ & $U$ \\
\hline$S$ & 1 & 0.86 & -0.15 \\
$T$ & 0.86 & 1 & -0.27 \\
$U$ & -0.15 & -0.27 & 1 \\
\hline \hline
\end{tabular}

Table 2: Correlation matrix for the fit to data for the variables $S, T$, and $U$ as described in the text.

large class of models, including new generations of fermions or modifications of the SM Higgs sector.

The dependence of various observables on the values of $S, T$, and $U$ is most easily calculated using the formalism developed by Kennedy and Lynn 43. Since this is a straightforward procedure [20], we will only list the results:

$$
\begin{aligned}
M_{W} & =80.35-0.29 S+0.45 T+0.34 U[\mathrm{GeV}], \\
\Gamma_{\ell^{+} \ell^{-}} & =83.98-0.18 S+0.78 T \quad[\mathrm{MeV}] \\
\sin ^{2} \theta_{\mathrm{eff}}^{\text {lept }} & =0.23179+0.00362 S-0.00258 T \\
g_{L}^{2} & =0.304-0.0027 S+0.0067 T \\
g_{R}^{2} & =0.030+0.0009 S-0.0002 T \\
g_{A}^{\nu e} & =-0.507-0.0037 T \\
g_{V}^{\nu e} & =-0.037+0.0068 S-0.0051 T \\
Q_{W}\left({ }_{55}^{133} \mathrm{Cs}\right) & =-73.2-0.74 S-0.007 T \\
Q_{W}\left({ }_{81}^{205} \mathrm{Tl}\right) & =-116.8-1.1 S-0.08 T
\end{aligned}
$$

By fitting these expressions to the data of Table1, we can obtain the experimentally preferred values of $S, T$, and $U$. We will only use the purely leptonic observables from LEP and SLC since their SM predictions are free from QCD uncertainties and are unaffected by possible direct corrections from new physics to the $Z b \bar{b}$ vertex. Using the ten data points for $M_{W}, \Gamma_{\ell^{+} \ell^{-}}, \sin ^{2} \theta_{\text {eff }}^{\text {lept }}(\mathrm{LEP}), \sin ^{2} \theta_{\mathrm{eff}}^{\text {lept }}(\mathrm{SLC}), g_{L}^{2}, g_{R}^{2}$, $g_{A}^{\nu e}, g_{V}^{\nu e}, Q_{W}\left({ }_{55}^{133} \mathrm{Cs}\right)$, and $Q_{W}\left({ }_{81}^{205} \mathrm{Tl}\right)$, we obtain the following:

$$
\begin{aligned}
& S=-0.33 \pm 0.19 \\
& T=-0.17 \pm 0.21 \\
& U=-0.34 \pm 0.50
\end{aligned}
$$

The correlation matrix between the variables in the fit is given in Table 2, and the quality of the fit is $\chi^{2}=4.5 /(10-3)$. The tightest constraints come from $\Gamma_{\ell^{+} \ell^{-}}$ and $\sin ^{2} \theta_{\text {eff }}^{\text {lept }}$. Indeed, fitting $S$ and $T$ to only the $\Gamma_{\ell^{+} \ell^{-}}$and $\sin ^{2} \theta_{\text {eff }}^{\text {lept }}$ data yields:

$$
\begin{aligned}
& S=-0.29 \pm 0.19 \\
& T=-0.13 \pm 0.22
\end{aligned}
$$


The central values of $S$ and $T$ are negative because both the $\Gamma_{\ell^{+} \ell^{-}}$and $\sin ^{2} \theta_{\text {eff }}^{\text {lept }}$ data are smaller than their SM predictions.

To give an example of how these bounds on $S, T$, and $U$ can provide important constraints on possible new physics sectors, consider the introduction of a new heavy fermion doublet $(N, E)$ with the usual left-handed couplings to $S U(2)_{L}$, hypercharge $Y$, and masses $m_{N}, m_{E} \gg M_{Z}$. The contribution of this doublet to $S, T$, and $U$ is given by

$$
\begin{aligned}
S= & \frac{1}{6 \pi}\left[1-Y \ln \left(\frac{m_{N}^{2}}{m_{E}^{2}}\right)\right] \\
T= & \frac{1}{16 \pi s^{2} c^{2} M_{Z}^{2}}\left[m_{N}^{2}+m_{E}^{2}-\frac{2 m_{N}^{2} m_{E}^{2}}{m_{N}^{2}-m_{E}^{2}} \ln \left(\frac{m_{N}^{2}}{m_{E}^{2}}\right)\right] \\
U= & \frac{1}{6 \pi}\left[-\frac{5 m_{N}^{2}-22 m_{N}^{2} m_{E}^{2}+5 m_{E}^{2}}{3\left(m_{N}^{2}-m_{E}^{2}\right)^{2}}\right. \\
& \left.\quad+\frac{m_{N}^{6}-3 m_{N}^{4} m_{E}^{2}-3 m_{N}^{2} m_{E}^{4}+m_{E}^{6}}{\left(m_{N}^{2}-m_{E}^{2}\right)^{3}} \ln \left(\frac{m_{N}^{2}}{m_{E}^{2}}\right)\right] .
\end{aligned}
$$

The above expression for $T$ is the usual contribution of a fermion doublet to the $\rho$ parameter and is positive semidefinite. Due to the constraint on $T$, this contribution must be constrained by keeping the mass splitting within the doublet to be small: i.e., $\Delta m \equiv\left|m_{N}-m_{E}\right| \ll m_{N}, m_{E}$. In this case, the above expressions simplify to

$$
\begin{aligned}
S & \approx \frac{1}{6 \pi} \approx 0.05 \\
T & \approx \frac{1}{12 \pi s^{2} c^{2}}\left[\frac{(\Delta m)^{2}}{M_{Z}^{2}}\right], \\
U & \approx \frac{2}{15 \pi}\left[\frac{(\Delta m)^{2}}{m_{N}^{2}}\right],
\end{aligned}
$$

and we see that we are now in conflict with the fitted value for $S$. Since the addition of each extra fermion doublet will contribute additively to $S, T$, and $U$, circumventing these limits quickly becomes a serious problem for theories such as technicolor where a large number of extra fermion doublets must be introduced. Furthermore, in the case of technicolor, strong interaction effects have been estimated to enhance the value of $S$ by roughly a factor of 2 [20].

Several suggestions have been made as to how one may introduce new fermions without conflicting with Eq. (21). Ref. [44] shows that Majorana fermions can simultaneously give negative contributions to both $S$ and $T$. Ref. 450 argues that if one introduces a complete generation of technifermions, keeping the techniquarks degenerate while splitting the masses of the technileptons will have the desired effect of making $S$ negative while keeping $T$ in check. Ref. 46 discusses the case where vectorlike, and mirror fermions are introduced. 
Many other interesting suggestions and models have been presented as to how one may extend the SM while conforming with the bounds on the values of $S$ and $T$ [47]. This has become increasingly difficult over the years due to the ever improving determination of $S$ and $T$. Calculating the values of $S$ and $T$ has now become a standard viability test for possible extensions of the SM.

\subsection{More Oblique Corrections}

Constraints on the values of $S, T$ and $U$, of course, do not apply to models that do not satisfy the three conditions discussed above. If we relax the third condition and allow the new physics scale to be near the weak scale, then more parameters must be introduced to express oblique corrections from new physics since the linear approximation of Eq. (17) no longer applies.

In Ref. [21], it was shown that it is sufficient to introduce three more parameters and increase the total number to six. Following the definition of Ref. [21], we slightly modify the previous definitions of $S$ and $U$ as follows and introduce three additional parameters $V, W$, and $X$. (The definition for $T$ remains unchanged.)

$$
\begin{aligned}
\alpha S & =4 s^{2} c^{2}\left[\frac{\Pi_{\mathrm{ZZ}}\left(M_{Z}^{2}\right)-\Pi_{\mathrm{ZZ}}(0)}{M_{Z}^{2}}-\frac{c^{2}-s^{2}}{s c} \Pi_{\mathrm{Z} \gamma}^{\prime}(0)-\Pi_{\gamma \gamma}^{\prime}(0)\right] \\
\alpha T & =\frac{\Pi_{\mathrm{WW}}(0)}{M_{W}^{2}}-\frac{\Pi_{\mathrm{ZZ}}(0)}{M_{Z}^{2}}, \\
\alpha U & =4 s^{2}\left[\frac{\Pi_{\mathrm{WW}}\left(M_{W}^{2}\right)-\Pi_{\mathrm{WW}}(0)}{M_{W}^{2}}-c^{2} \frac{\Pi_{\mathrm{ZZ}}\left(M_{Z}^{2}\right)-\Pi_{\mathrm{ZZ}}(0)}{M_{Z}^{2}}-2 s c \Pi_{\mathrm{Z} \gamma}^{\prime}(0)-s^{2} \Pi_{\gamma \gamma}^{\prime}(0)\right] \\
\alpha V & =\Pi_{\mathrm{ZZ}}^{\prime}\left(M_{Z}^{2}\right)-\frac{\Pi_{\mathrm{ZZ}}\left(M_{Z}^{2}\right)-\Pi_{\mathrm{ZZ}}(0)}{M_{Z}^{2}}, \\
\alpha W & =\Pi_{\mathrm{WW}}^{\prime}\left(M_{W}^{2}\right)-\frac{\Pi_{\mathrm{WW}}\left(M_{W}^{2}\right)-\Pi_{\mathrm{WW}}(0)}{M_{W}^{2}} \\
\alpha X & =-s c\left[\frac{\Pi_{\mathrm{Z} \gamma}\left(M_{Z}^{2}\right)}{M_{Z}^{2}}-\Pi_{\mathrm{Z} \gamma}^{\prime}(0)\right]
\end{aligned}
$$

In the limit $M_{\text {new }} \rightarrow \infty, S$ and $U$ coincide with their original definitions, and $V$, $W$, and $X$ vanish.

To place limits on $S$ through $X$, we must see how they alter the expressions for various observables. It turns out that the only relations in Eq. (20) that need to be modified are:

$$
\begin{aligned}
\Gamma_{\ell^{+} \ell^{-}} & =83.98-0.18 S+0.78 T+0.65 V-0.38 X \quad[\mathrm{MeV}] \\
\sin ^{2} \theta_{\text {eff }}^{\text {lept }} & =0.23179+0.00362 S-0.00258 T+0.00776 X
\end{aligned}
$$

The new parameter $W$ only appears in the $W$-width so we will not be considering it any further [21, 48]. Fitting these expressions to the same set of data as before, 


\begin{tabular}{|c||c|c|c|c|c|}
\hline \hline & $S$ & $T$ & $U$ & $V$ & $X$ \\
\hline$S$ & 1 & 0.79 & 0.48 & -0.76 & -0.94 \\
$T$ & 0.79 & 1 & -0.05 & -0.97 & -0.55 \\
$U$ & 0.48 & -0.05 & 1 & 0.05 & -0.68 \\
$V$ & -0.76 & -0.97 & 0.05 & 1 & 0.54 \\
$X$ & -0.94 & -0.55 & -0.68 & 0.54 & 1 \\
\hline \hline
\end{tabular}

Table 3: Correlation matrix for the fit to data for the six variables $S, T, U, V, W$, and $X$.

we obtain

$$
\begin{aligned}
& S=-1.0 \pm 1.5 \\
& T=-0.68 \pm 0.80 \\
& U=-0.21 \pm 0.92 \\
& V=0.56 \pm 0.83 \\
& X=0.13 \pm 0.51,
\end{aligned}
$$

with the correlation matrix given in Table 3 . The quality of the fit is $\chi^{2}=4.0 /(10-$ $5)$.

At first sight, the above results seem to indicate that the restrictions on the values of $S, T$ and $U$ are considerably relaxed. However, a careful look at the correlation matrix shows a large correlation between $\mathrm{T}$ and $\mathrm{V}$, and $\mathrm{S}$ and $\mathrm{X}$. So in order for $S$ and $T$ to deviate from their central values, they must be accompanied by corresponding shifts in $V$ and $X$. An easier way to see this is to redefine the parameters as

$$
\begin{aligned}
& S^{\prime}=S+4 s^{2} c^{2} V+4\left(c^{2}-s^{2}\right) X, \\
& T^{\prime}=T+V .
\end{aligned}
$$

Then, Eq. (26) will be reduced to

$$
\begin{aligned}
\Gamma_{\ell^{+} \ell^{-}} & =83.98-0.18 S^{\prime}+0.78 T^{\prime}[\mathrm{MeV}], \\
\sin ^{2} \theta_{\text {eff }}^{\text {lept }} & =0.23179+0.00362 S^{\prime}-0.00258 T^{\prime},
\end{aligned}
$$

and the same limits as those in Eq. (22) will apply to $S^{\prime}$ and $T^{\prime}$. Thus $Z$-pole observables alone can only probe the combination of $S^{\prime}$ and $T^{\prime}$. Therefore, to accommodate large deviations of $S$ and $T$ from their central values, the model must also predict large values for $V$ and $X$. While it is possible to construct exceptional cases where $V$ and $X$ are as large as or larger than $S$ or $T$ 49], in most cases of interest they are still much smaller than $S$ or $T$ due to the natural suppression factor $M_{Z}^{2} / M_{\text {new }}^{2}$ [50].

It is interesting to note that new physics may be quite close to the weak scale and yet makes little contribution to the oblique parameters. As an example, we 
consider the low-energy sector of the string-inspired SUSY- $E_{6}$ model wherein the ordinary particle spectrum of the MSSM is augmented by three generations of exotic fermions and their SUSY partners as described in Section 2. If, for simplicity, we ignore mixing between these states and the SM fields (and we also take them to be degenerate), we find that the new contribution to $T$ vanishes automatically due to the vector-like nature of the exotic fields. The corresponding contributions to the other oblique parameters are shown in Fig. 1 and are seen to be small $(\lesssim 0.1)$ for $m \gtrsim 150 \mathrm{GeV}$. This example shows that new physics may be lurking nearby without it showing up in the oblique corrections.

Figure 1: Contribution of 3 generations of degenerate $E_{6}$ exotic fermions of mass $M$ and their SUSY partners to the oblique parameters. From top to bottom the curves correspond to the parameter $-V,-W,-S, X$, and $-U$, respectively.

\subsection{Non-Oblique Corrections}

Let us now turn to the problem of constraining non-oblique direct corrections. There has been considerable attention to non-oblique corrections due to the $3.8 \sigma$ and $2.4 \sigma$ discrepancy between the SM and LEP/SLC values of $R_{b}$ and $R_{c}$. The dependence of these observables on oblique corrections (which is already severly constrained by $S$ and $T$ ) is weak, so this discrepancy must be explained by extra non-oblique corrections from new physics. Many models of new physics do predict a large correction to the $Z b \bar{b}$ vertex [51] and hence to $R_{b}$ but often in the direction

of making the discrepancy even larger. It is therefore useful to introduce parameters which describe these non-oblique corrections in a model independent fashion 
and constrain then with the data to facilitate the comparison between theory and experiment.

Here we will use the formalism of Ref. [22]; we note that a similar formalism was developed earlier in Ref. [52]. We will limit ourselves to the situation where the only relevant non-oblique correction from new physics is in the $Z b \bar{b}$ vertex. We express corrections from new physics to the effective left- and right-handed couplings of the $b$ quark to the $Z$ as

$$
\begin{aligned}
& g_{L}^{b}=\left[g_{L}^{b}\right]_{\mathrm{SM}}+\frac{1}{3} \delta s^{2}+\delta g_{L}^{b}, \\
& g_{R}^{b}=\left[g_{R}^{b}\right]_{\mathrm{SM}}+\frac{1}{3} \delta s^{2}+\delta g_{R}^{b},
\end{aligned}
$$

where $\delta s^{2}$ expresses the shift of the effective weak angle due to oblique corrections, and $\delta g_{L}^{b}$ and $\delta g_{R}^{b}$ express non-oblique vertex corrections from new physics. In terms of $S$ and $T, \delta s^{2}$ is given by:

$$
\delta s^{2}=\frac{\alpha}{c^{2}-s^{2}}\left[\frac{1}{4} S-s^{2} c^{2} T\right] .
$$

It is convenient in practice to define the following linear combinations of $\delta g_{L}^{b}$ and $\delta g_{R}^{b}$ :

$$
\begin{aligned}
\xi_{b} & \equiv\left(\cos \phi_{b}\right) \delta g_{L}^{b}-\left(\sin \phi_{b}\right) \delta g_{R}^{b} \\
\zeta_{b} & \equiv\left(\sin \phi_{b}\right) \delta g_{L}^{b}+\left(\cos \phi_{b}\right) \delta g_{R}^{b}
\end{aligned}
$$

where

$$
\phi_{b} \equiv \tan ^{-1}\left|g_{R}^{b} / g_{L}^{b}\right|=0.182,
$$

since $\Gamma_{b \bar{b}}$ depends only on the linear combination $\xi_{b}$, while $A_{b}$ depends only on the linear combination $\zeta_{b}$.

The dependence of the relevant observables on $\delta s^{2}, \xi_{b}$, and $\zeta_{b}$ are can be calculated just as in the $S, T, U$ case and we find

$$
\begin{aligned}
\sigma_{\mathrm{h}}^{0} & =41.454+4.4 \delta s^{2}+17 \xi_{b}-5.1 \delta \alpha_{s} \quad[\mathrm{nb}] \\
R_{\ell} & =20.767-18 \delta s^{2}-21 \xi_{b}+6.4 \delta \alpha_{s}, \\
R_{b} & =0.2155+0.04 \delta s^{2}-0.78 \xi_{b}, \\
R_{c} & =0.1724-0.06 \delta s^{2}+0.18 \xi_{b}, \\
A_{\mathrm{FB}}^{0, b} & =0.1016-5.6 \delta s^{2}-0.18 \zeta_{b}, \\
A_{b} & =0.9345-0.64 \delta s^{2}-1.6 \zeta_{b}, \\
\sin ^{2} \theta_{\mathrm{eff}}^{\text {lept }} & =0.23179+\delta s^{2} .
\end{aligned}
$$

For $\sigma_{\mathrm{h}}^{0}$ and $R_{\ell}$ we have also included a correction due to the shift of the QCD coupling constant from its reference value: $\alpha_{s}\left(M_{Z}\right)=0.123+\delta \alpha_{s}$. We will not 


\begin{tabular}{|c||c|c|c|c|}
\hline \hline & $\delta s^{2}$ & $\xi_{b}$ & $\zeta_{b}$ & $\delta \alpha_{s}$ \\
\hline$\delta s^{2}$ & 1 & 0.02 & -0.47 & 0.11 \\
$\xi_{b}$ & 0.02 & 1 & 0.06 & 0.87 \\
$\zeta_{b}$ & -0.47 & 0.06 & 1 & 0.02 \\
$\delta \alpha_{s}$ & 0.11 & 0.87 & 0.02 & 1 \\
\hline \hline
\end{tabular}

Table 4: Correlation matrix for the fit to the variables in Eq. (35).

be considering $\Gamma_{Z}$ in our analysis since it has an extra dependence on oblique corrections coming from the $\rho$ parameter which is absent in the partial width ratios $R_{\ell}, R_{b}$, and $R_{c}$, as well as $\sigma_{\mathrm{h}}^{0}$,

Fitting the above expressions to the LEP/SLC values of $\sigma_{\mathrm{h}}^{0}, R_{\ell}, R_{b}(\mathrm{LEP})$, $R_{b}(\mathrm{SLC}), R_{c} A_{\mathrm{FB}}^{0, b}, A_{b}, \sin ^{2} \theta_{\mathrm{eff}}^{\text {lept }}(\mathrm{LEP})$ and $\sin ^{2} \theta_{\mathrm{eff}}^{\text {lept }}(\mathrm{SLC})$ we find

$$
\begin{aligned}
\delta s^{2} & =-0.00084 \pm 0.00034, \\
\xi_{b} & =-0.006 \pm 0.002, \\
\zeta_{b} & =0.035 \pm 0.017, \\
\delta \alpha_{s} & =-0.023 \pm 0.008,
\end{aligned}
$$

with the correlation matrix in Table $甘$. The quality of the fit is $\chi^{2}=9.8 /(9-4)$ with $R_{c}$ alone contributing 6.0 to the overall $\chi^{2}$. This means that $R_{c}$ still has a $2.4 \sigma$ discrepancy between theory and experiment even with extra corrections to the $Z b \bar{b}$ vertex. In order to explain this discrepancy, we must introduce extra nonoblique corrections to the $Z c \bar{c}$ and perhaps other vertices as well. (A most general fit including corrections to all the vertices has been performed in Ref. [53.)

Assuming that the discrepancy in $R_{c}$ is just statistical for the moment, we shall ignore it and convert the bounds on $\xi_{b}$ and $\zeta_{b}$ into constraints on the original $\delta g_{L}^{b}$ and $\delta g_{R}^{b}$. We find:

$$
\begin{aligned}
\delta g_{L}^{b} & =0.0004 \pm 0.0037 \\
\delta g_{R}^{b} & =0.036 \pm 0.017
\end{aligned}
$$

with a correlation of 0.84 . Again, due to this large correlation, care is needed when comparing these limits with theory. For instance, many theories beyond the SM predict $\delta g_{R}^{b} \approx 0$ (such as Supersymmetry) in which case the limit on $\delta g_{L}^{b}$ becomes

$$
\delta g_{L}^{b}=-0.006 \pm 0.002 \quad\left(\delta g_{R}^{b}=0\right),
$$

and the disagreement between the SM and experiment is at the $3 \sigma$ level.

In order to explicitly display the discrepancy with the SM, Fig. 2 shows the result of fitting the values of $\delta g_{L, R}^{b}$ to the $Z \rightarrow b \bar{b}$ data set presented in Table 1. 
For simplicity, we now define

$$
\begin{aligned}
g_{L}^{b} & =-\frac{1}{2}+\frac{1}{3} \sin ^{2} \theta_{w}^{b}+\delta g_{L}^{b}, \\
g_{R}^{b} & =\frac{1}{3} \sin ^{2} \theta_{w}^{b}+\delta g_{R}^{b},
\end{aligned}
$$

where $\sin ^{2} \theta_{w}^{b}$ is the b-quark effective weak mixing angle, and use ZFITTER4.9 to calculate the SM predictions for different values of $m_{t}$. (Here, we take $m_{H}=$ $300 \mathrm{GeV}, \alpha_{s}\left(M_{Z}\right)=0.125$, and $\alpha_{e m}^{-1}\left(M_{Z}\right)=128.896$, but our results are quite insensitive to this particular choice of parameters.) As can be seen from the figure, one gains more in $\chi^{2}$ if we allow $\delta g_{R}^{b} \neq 0$. We note in passing that the SM point remains outside of the region selected by the data even if we increase the C.L. to $99.9 \%$.

We now discuss the influence of some specific models on $R_{b}$. A recent summary of the effects of several classes of new physics on $R_{b}$ can be found in Ref. [51].

Figure 2: $95 \%$ C.L. fit to the parameters $\delta g_{L, R}^{b}$ using the full LEP/SLC $Z \rightarrow b \bar{b}$ data set and ZFITTER4.9. The dashed (solid, dotted) curve corresponds to $m_{t}=170(180,190)$ $\mathrm{GeV}$. The diamond at the center is the SM prediction. The three nearby diamonds are the $\chi^{2}$ minima for $m_{t}=170,180,190 \mathrm{GeV}$ (from top to bottom). The values of the other input parameters are as given in the text.

\subsection{1 $R_{b}$ and Supersymmetry}

Supersymmetric models with light super-partners could potentially account for the $R_{b}$ anomaly. There are two well-separated regions [54 of supersymmetric 
parameter space where large corrections to the $Z b \bar{b}$ vertex are possible: low $\tan \beta \simeq$ 1 , and high $\tan \beta>50$. This is illustrated in Fig. 3. The low $\tan \beta$ region makes use of the large top Yukawa entering in the $\tilde{t}_{R}-\tilde{H}^{+}-b$ and $t-H^{+}-b$ interactions. The high $\tan \beta$ region makes use of the large bottom Yukawa $\left(m_{b} / \cos \beta\right)$ entering in the $\tilde{b}-\tilde{H}^{0}-b$ and $b-b-A$ interactions. In both cases, the overall effect is to increase the theoretical prediction of $R_{b}$ over the standard model value and bring theory more in line with experiment. However, it has been argued in [55] that the high $\tan \beta$ region is not a viable solution to the $R_{b}$ anomaly because it violates experimental constraints on $Z \rightarrow b \bar{A}$ [56] and $b \rightarrow c \tau \nu_{\tau}$ [57] decay data. Fig. [t graphically demonstrates the exclusion of high $\tan \beta$ models. Only the low $\tan \beta$ region is allowed.

Figure 3: The dependence of $R_{b}^{\max }$ on $\tan \beta$. The maximum possible value for $R_{b}$ obtainable as a function of $\tan \beta$ is plotted for $m_{\chi_{1}^{ \pm}}=46 \mathrm{GeV}$ (upper line) and $m_{\chi_{1}^{ \pm}}=$ $60 \mathrm{GeV}$ (lower line). The upper hatched region is the experimental $1 \sigma$ range for $R_{b}$, while the lower range represents the SM range consistent with the $1 \sigma$ bounds for $m_{t}$. Only the very low and very high values of $\tan \beta$ can significantly enhance the theoretical prediction for $R_{b}$.

A successful low $\tan \beta$ supersymmetric model will have a light Higgsino-like chargino, and a light top squark which is mainly the super-partner of the righthanded top quark. These requirements follow directly from the need to maximize the coupling of the charginos and stops to the bottom quarks. The mass of the chargino and stop must be below approximately $80 \mathrm{GeV}$ (as shown in Fig. : to keep the loop integral from being suppressed. Additional phenomenological implications arise from such models [55, 58, 59]. For example, the branching ratio 
of the top quark to top squark plus neutralinos is at or above 0.4. Although not currently excluded by Tevatron data, this large branching fraction of top decays into particles other than $b+W^{+}$should be noticeable in the very near future.

LEP2 should be able to find these light sparticles in pair production roughly up to its kinematic limit. In fact, preliminary results from the recent LEP 1.5 run in November 1995 places[3] lower bounds on the chargino mass of $\gtrsim 65 \mathrm{GeV}$, if the chargino - lightest neutralino mass splitting is greater than $10 \mathrm{GeV}$. This null search clearly makes it more difficult for SUSY alone to accommodate the experimental value of $R_{b}$ [60]. Perhaps larger SUSY GUT groups, such as SO(10) or $E_{6}$, with a correspondingly larger particle content should be investigated.

It is crucial to test that these models of supersymmetry which yield large shifts in $R_{b}$ do not violate other constraints. Most importantly, one could imagine that light superpartners inducing large vertex corrections could also substantially alter other precision data constraints, such as the $\rho$ parameter. It has been shown by several groups [61, 62, 63, 64] that the global fit of electroweak observables in supersymmetry not only allows models with higher $R_{b}$ but fits the compendium of data better than that standard model as measured by total $\chi^{2}$.

As previously discussed, a discrepancy of $2.4 \sigma$ between the experimental and SM predicted value of $R_{c}$ has also been reported. Supersymmetry has no natural way to explain such a large deviation in $R_{c}[63$, and so its "true value" is usually fixed to that of the SM value to maintain consistency in supersymmetry analyses. In addition to a possible statistical fluctuation in the measurement of $R_{c}$ and the large correlation of the measurement to $R_{b}$, another compelling reason why the $R_{c}$ discrepancy might not be real is its correlation with $\alpha_{s}$. Recall that additional physics which brings the $R_{b}$ prediction closer to experiment also has the effect of lowering the extracted $\alpha_{s}$ from the $Z$ line shape analysis - a welcome development since the $Z$ line shape $\alpha_{s}$ in the standard model is higher than what other methods seem to indicate. If both $R_{b}$ and $R_{c}$ were to be accounted for by new physics, and their true values were fixed at the present measured central values, then the extracted $\alpha_{s}$ from the $Z$ line shape would actually go up, making it even more discordant with low energy $\alpha_{s}$ extractions.

Another observable, the $b$-quark asymmetry $A_{b}=\left(g_{L}^{2}-g_{R}^{2}\right) /\left(g_{L}^{2}+g_{R}^{2}\right)$, is also demonstrating $\mathrm{a} \sim 2 \sigma$ deviation from the standard model prediction. Given a supersymmetric spectrum which produces a large shift in the $Z b \bar{b}$ couplings sufficient to predict $R_{b}$ closer to the data, perhaps these light superpartners would induce a more harmonious $A_{b}$ prediction as well. A closer look shows this not to be the case. In fact, the supersymmetry prediction is indistinguishable from the standard model case. The reason is that shifts in $R_{b}$ are most sensitive to corrections to the left-handed coupling of the $b$-quarks to the $Z$, while the shifts in $A_{b}$ are most sensitive to corrections to the right-handed couplings. But supersymmetry can only significantly change the left-handed coupling; therefore, consequential shifts of $A_{b}$ in supersymmetry model are not expected. 
A common framework employed in the study of supersymmetry phenomenology imposes gauge coupling unification, common scalar masses at the high scale (GUT scale or Planck scale), common gaugino masses at the high scale, etc. 65. This framework, sometimes called a "super-unified" model, is motivated by the apparent meeting of the gauge couplings at the high scale, minimal supergravity boundary conditions from simple SUSY breaking paradigms, successful description of radiative electroweak symmetry breaking, flavor changing neutral current constraints on the squark and slepton masses, and more. It turns out that the masses and mixings required to yield a large result in $R_{b}$ are not exhibited in these superunified models, and a more general low energy Lagrangian framework must be adopted [66, 67]. A natural connection between models yielding "good $R_{b}$ " at the electroweak scale to more fundamental models at the high scale is still unresolved, and perhaps will remain so until additional observables weigh in.

Figure 4: The high $\tan \beta$ exclusion plot as argued by Ref. [55]. The $\delta R_{b}=0.003$ contour is such that no supersymmetric solution below the contour can provide $\delta R_{b} \geq 0.003$. The region above the $r=\tan \beta / m_{H^{ \pm}}=0.52 \mathrm{GeV}^{-1}$ contour is excluded by $b \rightarrow c \tau \nu_{\tau}$ decay data. The region to the left of the vertical lines, which indicate contours of $Z \rightarrow b \bar{b} A$ events, is perhaps already excluded by current data as argued in the above reference. Therefore, if one requires $\delta R_{b}>0.003$ then no region of the high $\tan \beta$ parameter space is simultaneously consistent with the $b \rightarrow c \tau \nu_{\tau}$ and $Z \rightarrow b \bar{b} A$ decay constraints.

\subsection{2 $\quad R_{b}$ and Extended Technicolor}

In extended 68] technicolor 69] (ETC) models, the large mass of the top quark generally arises from ETC dynamics at relatively low energy scales. Since the magnitude of the Cabibbo-Kobayashi-Maskawa (CKM) matrix element $\left|V_{t b}\right|$ is nearly 
Figure 5: Contour of $\delta R_{b}=0.003$ in the $m_{\chi_{1}^{ \pm}}-m_{\tilde{t}_{1}}$ plane with $m_{t}=170 \mathrm{GeV}$ and $\tan \beta=$ 1.1. Above the contour no solution exists which yields $\delta R_{b}>0.003$. Below the contour solutions do exist with $\delta R_{b}>0.003$ for appropriate choices of parameters. The numerical value of this contour is enhanced (or diminished) by about $(0.4 / \sin \beta)^{2}\left(m_{t} / M_{Z}\right)^{2}$ for different choices of $m_{t}$ and $\tan \beta$.

unity, $S U(2)_{L}$ gauge invariance insures that ETC bosons coupling to the lefthanded top quark couple with equal strength to the left-handed bottom quark. In particular, the ETC dynamics which generate the top quark's mass also couple to the left-handed bottom quark thereby affecting the $Z b \bar{b}$ vertex. This has been shown 70 to provide a powerful experimental constraint on extended technicolor models - particularly on those models in which the ETC gauge group commutes with $S U(2)_{L}$.

Consider a model in which $m_{t}$ is generated by the exchange of a weak-singlet ETC gauge boson of mass $M_{E T C}$ coupling with strength $g_{E T C}$ to the current

$$
\xi \bar{\psi}_{L}^{i} \gamma^{\mu} T_{L}^{i k}+\frac{1}{\xi} \bar{t}_{R} \gamma^{\mu} U_{R}^{k}, \quad \text { with } \psi_{L} \equiv\left(\begin{array}{l}
t \\
b
\end{array}\right)_{L} T_{L} \equiv\left(\begin{array}{l}
U \\
D
\end{array}\right)_{L},
$$

where $U$ and $D$ are technifermions, $i$ and $k$ are weak and technicolor indices, and $\xi$ is an ETC Clebsch expected to be of order one. At energies below $M_{E T C}$, ETC gauge boson exchange may be approximated by local four-fermion operators. For example, $m_{t}$ arises from an operator coupling the left- and right-handed currents in Eq. (39)

$$
-\frac{g_{E T C}^{2}}{M_{E T C}^{2}}\left(\bar{\psi}_{L}^{i} \gamma^{\mu} T_{L}^{i w}\right)\left(\bar{U}_{R}^{w} \gamma_{\mu} t_{R}\right)+\text { h.c. . }
$$

Assuming, for simplicity, that there is only a doublet of technifermions and that technicolor respects an $S U(2)_{L} \times S U(2)_{R}$ chiral symmetry (so that the technipion decay constant, $F$, is $v=246 \mathrm{GeV}$ ), the rules of naive dimensional analysis 71 
give an estimate of

$$
m_{t}=\frac{g_{E T C}^{2}}{M_{E T C}^{2}}\langle\bar{U} U\rangle \approx \frac{g_{E T C}^{2}}{M_{E T C}^{2}}\left(4 \pi v^{3}\right)
$$

for the top quark mass when the technifermions' chiral symmetries break.

The ETC boson responsible for producing $m_{t}$ also affects the $Z b \bar{b}$ vertex 70 when exchanged between the two left-handed fermion currents of Eq. (39). This diagram alters the $Z$-boson's tree-level coupling to left-handed bottom quarks by

$$
\delta g_{L}^{E T C}=-\frac{\xi^{2}}{2} \frac{g_{E T C}^{2} v^{2}}{M_{E T C}^{2}} \frac{e}{\sin \theta \cos \theta}\left(I_{3}\right)=\frac{1}{4} \xi^{2} \frac{m_{t}}{4 \pi v} \frac{e}{\sin \theta \cos \theta},
$$

where the right-most expression follows from applying Eq. (41), and $\theta$ is the weak mixing angle. The ETC-induced shift in $R_{b}$ is then 70

$$
\frac{\delta R_{b}}{R_{b}} \approx-5.1 \% \xi^{2}\left(\frac{m_{t}}{175 \mathrm{GeV}}\right)
$$

which is large enough to be detected by the LEP experiments. Since the experimental value of $R_{b}$ actually lies above the SM prediction, any contribution from non-standard physics is positive: i.e., $\left[\delta R_{b} / R_{b}\right]_{\text {new }} \approx+3 \%$, thereby excluding ETC models in which the ETC and weak gauge groups commute.

It is also interesting to check how more realistic ETC models fare. The following summary indicates how the $Z b \bar{b}$ vertex can guide model builders.

- A slowly-running ('walking') technicolor beta-function is included in ETC models to provide the light fermions with large enough masses, while avoiding excessive flavor-changing neutral currents [72. Because a walking beta function enhances the technifermion condensate $\langle\bar{T} T\rangle$, it leads to larger fermion masses for a given ETC scale, $M_{E T C}$. Enhancing $m_{t}$ relative to $M_{E T C}$ does reduce the size of $\delta g_{L}-$ but, generally, not enough to render the shift in $R_{b}$ invisible to LEP [73].

- In some ETC models, the ETC coupling itself becomes strong before the scale $M_{E T C}$ and plays a significant role in electroweak symmetry breaking [74. The spectra of strongly-coupled ETC models include light composite scalars with Yukawa couplings to ordinary fermions and technifermions [75] . Exchange of the composite scalars produces corrections to $R_{b}$ that are allowed by experiment [76]. The disadvantage of this approach is the need to fine-tune the ETC coupling close to the critical value.

- ETC models also generally include 'diagonal' techni-neutral ETC bosons. The effect of these gauge bosons on $R_{b}$ is discussed at length in Ref. [77. Suffice it to say that while exchange of the diagonal ETC bosons does tend to raise $R_{b}$, this effect is significant only when the model includes large isospin violation - leading to conflict with the measured value of the oblique parameter $T$. 
- Finally, we should recall that our analysis explicitly assumed that the weak and ETC gauge groups commute. More recent work [78 indicates that models in which ETC gauge bosons carry weak charge can give experimentally allowed values of $R_{b}$ due to cancellation between two contributions to the $Z b \bar{b}$ vertex (from exchange of the ETC boson that generates $m_{t}$ and from $Z-Z^{\prime}$ mixing). Furthermore, these non-commuting ETC models can actually fit the full set of precision electroweak data better than the standard model!

\subsection{3 $\quad R_{b}$ and Anomalous Couplings}

The possibility that the conventional SM $Z b \bar{b}$ vertex is modified due to the presence of weak electric $\left(\tilde{\kappa}_{b}\right)$ and/or magnetic $\left(\kappa_{b}\right)$ anomalous moment type couplings 77 , 80] has been considered as a possible explanation [81] of the high measured value of $R_{b}$. Specifically, the $Z b \bar{b}$ interaction now takes the form

$$
\mathcal{L}=\frac{g}{2 c_{w}} \bar{b}\left[\gamma_{\mu}\left(v_{b}-a_{b} \gamma_{5}\right)+\frac{i}{2 m_{b}} \sigma_{\mu \nu} q^{\nu}\left(\kappa_{b}-i \tilde{\kappa}_{b} \gamma_{5}\right)\right] f Z^{\mu},
$$

where $g$ is the standard weak coupling constant, $c_{w}=\cos \theta_{W}, m_{b}$ is the $b$-quark mass, $v_{b}\left(a_{b}\right)$ are the SM couplings and $q$ is the $Z$ 's four-momentum. Note that the weak electric dipole moment coupling is $C P$-violating. If $\tilde{\kappa}_{b}$ and/or $\kappa_{b}$ were nonzero, $R_{b}$ and $A_{F B}^{b}$ (the forward-backward asymmetry), as well as $A_{p o l}^{b}$ (the polarized forward-backward asymmetry), which can only be measured by SLD, would be found to differ from the expectations of the SM. A description of all these observables and their dependencies on $\tilde{\kappa}$ and $\kappa$ is given in detail in Ref. 81. Using the data presented at the EPS95 and Beijing summer conferences[24], Fig. 6] displays the effects of these anomalous couplings. In particular, it shows the scaled ratios $R_{b} / R_{b}^{S M}$ and $A_{b} / A_{b}^{S M}$, where the latter quantity is the weighted combination of $A_{F B}^{b} / A_{F B}^{b}(S M)$ and $A_{p o l}^{b} / A_{p o l}^{b}(S M)$. Note that $R_{b}\left(A_{b}\right)$ is increased(decreased) by the existence of anomalous couplings. In this analysis we take $\alpha_{s}\left(M_{Z}\right)=0.125$, $\alpha_{e m}^{-1}\left(M_{Z}\right)=128.896$, and the SM Higgs boson mass $\left(m_{H}\right)$ was set to $300 \mathrm{GeV}$. A modified version of ZFITTER4.9 31] was used to obtain the predictions of the SM for these observables. Note that the presence of the anomalous couplings push the $\mathrm{SM}$ in the general direction of the data!

Allowing $\tilde{\kappa}_{b}$ and $\kappa_{b}$ to be non-zero, a $\chi^{2}$ fit was performed to determine the $95 \%$ CL region for these anomalous couplings as shown in Fig. 7. The SM lies outside the boundary of the allowed region due to the $>3 \sigma$ discrepancy in the value of $R_{b}$ and the somewhat low $(\simeq 1.8 \sigma)$ value of $A_{b}$ from SLD. The data clearly prefers non-zero anomalous couplings. Performing the corresponding analyses for charm quarks and $\tau$ leptons yields the shown results in Fig. 8. In the charm-quark case, $R_{c}$ is below the SM prediction while anomalous couplings can only produce an increase in $R_{c}$ thus leading to very strong constraints. The $\tau$ data on the otherhand is in complete agreement with the SM predictions which also produces tight bounds on the corresponding anomalous couplings. 
Figure 6: $R_{b}$ vs. $A_{b}$ compared with the predictions of the SM for $m_{t}=170,180,190$ $\mathrm{GeV}$, corresponding to the dotted, solid, dashed data point, respectively. The upper(lower) solid curve is the prediction for non-zero negative(positive) values of $\kappa_{b}$ with the points in steps of 0.01 . The dashed line represents the corresponding case of non-zero $\tilde{\kappa}_{b}$.

Figure 7: Regions in the $\kappa_{b}-\tilde{\kappa}_{b}$ plane allowed at the $95 \%$ CL by the Beijing and EPS95 data for $m_{t}=170,180,190 \mathrm{GeV}$, corresponding to the inside of the dotted, solid, and dashed curves, respectively. The diamonds mark the corresponding $\chi^{2}$ minima from left to right. 
Figure 8: Allowed values of the anomalous couplings for charm and $\tau$ for the same top masses as in Fig.2.

Figure 9: Shift in the value of $x_{w}^{\text {eff }}=\sin ^{2} \theta_{\text {eff }}^{\text {leptons }}$ extracted from the LEP determination of the $\tau$ polarization, $P_{\tau}$, due to non-zero values of $\kappa_{\tau}^{Z}$ (solid) or $\tilde{\kappa}_{\tau}^{Z}$ (dotted). 
Anomalous couplings could lead to interesting results elsewhere. Although we obtain reasonably strong constraints on the anomalous couplings of the $\tau$ from precision measurements, it is interesting to contemplate how such couplings might influence the values extracted for $x_{w}^{e f f}=\sin ^{2} \theta_{\text {eff }}^{\text {leptons }}$ from various observables involving $\tau$ 's. As an example, we consider the case of the $\tau$ polarization asymmetry, $P_{\tau}$. Fig. 9 shows how non-zero values of either $\kappa_{\tau}^{Z}$ or $\tilde{\kappa}_{\tau}^{Z}$ can lead to an apparent shift in the value of $x_{w}^{e f f}$ extracted from $P_{\tau}$. If $\kappa_{\tau}^{Z}=0.002$ then $\delta x_{w}^{e f f}=0.001$, which means that the true value of $x_{w}^{e f f}$ is 0.001 lower than what would be extracted by naively using the SM formulae. It is interesting to note that the experimental value of $x_{w}^{e f f}$ extracted from $P_{\tau}$ is somewhat higher than that given by either the leptonic forward-backward asymmetries or $A_{L R}$.

\subsection{Extra Gauge Bosons}

When the electroweak gauge group is extended beyond the standard $S U(2)_{L} \times$ $U(1)_{Y}$, and new electroweak gauge bosons such as extra $Z^{\prime}$ s and/or right-handed $W$ 's are introduced, then one must consider corrections that are not encompassed in the usual oblique and non-oblique correction framework. These corrections are due to the direct exchange of the new bosons between the external fermions, and due to mixing among the new and ordinary gauge bosons, which will affect their masses and couplings. These corrections enter at both tree-level and at 1-loop. There are also additional loop corrections from the new gauge bosons as well as from extra fermions that must be introduced for anomaly cancellation purposes, and a more complicated Higgs sector necessary for giving masses to all the gauge bosons except the photon, will all come into the picture. This makes the analysis of tree-level and 1-loop radiative corrections from models with extra gauge bosons extremely complicated and difficult to discuss in any simple model independent way[ [6].

However, a restricted $S-T$ type of analysis is possible 82, 83 if we limit ourselves to only leptonic observables at the $Z$ pole and $M_{W}$. In this case since there are only three observables under consideration, one is completely free to parameterize their potential deviations from SM predictions in terms of three variables which can be identified as $S, T$, and $U$. The following approximate relations for the shifted values of $S, T$, and $U$ due to $Z^{\prime}$ exchange can be obtained (the exact relations are rather cumbersome and can be found in Ref. [82]):

$$
\begin{aligned}
& \alpha \Delta S \simeq 2 \phi\left[\left(1-2 x_{w}\right) v^{\prime}-\left(1+2 x_{w}\right) a^{\prime}\right] \\
& \alpha \Delta T \simeq\left(\delta \rho-4 a^{\prime} \phi\right) \\
& \alpha \Delta U \simeq 4 \phi\left(v^{\prime}+3 a^{\prime}\right)
\end{aligned}
$$

where $v^{\prime}$ and $a^{\prime}$ are the charged lepton couplings to the $Z^{\prime}$ (normalized as in the $\mathrm{SM}), \phi$ is the $Z-Z^{\prime}$ mixing angle, and $\delta \rho$ represents the shift in the effective $\rho$ 
parameter due to the $Z^{\prime}$. $\delta \rho, \phi, v^{\prime}$, and $a^{\prime}$ are easily calculable within a specific extended gauge theory [6, 83]. It is possible to obtain consistency [83] with the data in several models, including $E_{6}$ GUTS and the LRM, with $Z^{\prime}$ masses below 1 $\mathrm{TeV}$. It is important to remember here that all $Z$-pole and $M_{W}$ effects are due to $Z-Z^{\prime}$ mixing. Hence we also note here that general global electroweak analyses restrict 84 the $Z-Z^{\prime}$ mixing angle to be $|\phi| \lesssim 0.01$, which can in turn provide model dependent bounds on the $Z^{\prime}$ mass [85].

The sensitivity of the weak charge $Q_{W}$, as determined in atomic parity violation experiments, to the existence of a $Z^{\prime}$ has been discussed by several authors [86. It is found that (see also Ref. [83]) one of the best signatures of a $Z^{\prime}$ arising from $E_{6}$ or the LRM is a small positive increase in the value for the weak charge in Cesium, $\delta Q_{W} \simeq 0.2-0.3$, in comparison to the SM prediction. These effects can be important even if $Z-Z^{\prime}$ mixing is absent, in contrast to the $Z$-pole data discussed above. Future experiments are expected to be sensitive to these effects.

\section{A Model-Independent Global Analysis}

A useful simplification occurs whenever all of the new particles which arise in a model are heavy compared to the energies which are accessible in the experiments of interest. In this case all of the model's predictions for these experiments can be summarized by an effective Lagrangian, in which all of the heavy particles are 'integrated out'. The resulting effective interactions amongst the light particles describe the virtual effects of all of the heavy particles. Since the coefficients of higher-dimension interactions are suppressed by higher powers of the inverse of the heavy-particle masses in a computable way, this technique of organizing calculations underlines the fact that only a comparatively few combinations of the parameters of the model can contribute to low-energy observables.

These observations suggest a more model-independent way to explore the implications for new physics of current experiments. The approach is based on the observation that all models which share the same low-energy particle content are described by the same low-energy Lagrangian, differing only through the couplings they predict for each of the possible effective interactions. The predictions which are common to all such models may be obtained by working with the most general possible effective Lagrangian which is allowed by the low-energy particle content and symmetries, but using completely arbitrary couplings. The price to be paid for the model-independence of the resulting predictions is the loss of the predictive power which is possible when the effective Lagrangian is derived from a particular model. The following discussion summarizes the constraints which may

be obtained from precision electroweak measurements by pursuing this type of model-independent approach. 


\subsection{The Lowest-Dimension Effective Interactions}

We start with the most general possible effective Lagrangian which involves only the known light particles - taken in what follows to be the SM complement, excluding the top quark and Higgs boson - and which respects electromagnetic gauge invariance. For the present purposes, the effective couplings of the QCD gluons may be ignored. Working up to and including mass dimension five, one finds that the most important terms can be cast into the following form 87]:

1. Electromagnetic Couplings: The electromagnetic couplings of fermions are

$$
\mathcal{L}_{\mathrm{em}}=-e\left[\bar{f}_{i} \gamma^{\mu} Q_{i} f_{i} A_{\mu}+\bar{f}_{i} \sigma^{\mu \nu}\left(d_{L}^{i j} \gamma_{L}+d_{R}^{i j} \gamma_{R}\right) f_{j} F_{\mu \nu}\right],
$$

where the indices $i$ and $j$ are to be summed over all possible flavors of light fermions, $f_{i}$. Here $\gamma_{L}$ and $\gamma_{R}$ denote the usual projection matrices onto left- and righthanded spinors. The effective couplings, $d_{L}^{i j}$ and $d_{R}^{i j}$, represent linear combinations of nonstandard magnetic- and electric-dipole moment interactions.

2. Charged-Current Interactions: The fermion charged-current interactions become

$$
\begin{aligned}
\mathcal{L}_{\mathrm{cc}}=-\frac{e}{\sqrt{2} s_{w}} & {\left[\bar{f}_{i} \gamma^{\mu}\left(h_{L}^{i j} \gamma_{L}+h_{R}^{i j} \gamma_{R}\right) f_{j} W_{\mu}^{\dagger}\right.} \\
& \left.+\bar{f}_{i} \sigma^{\mu \nu}\left(c_{L}^{i j} \gamma_{L}+c_{R}^{i j} \gamma_{R}\right) f_{j} W_{\mu \nu}^{\dagger}\right]+ \text { h.c. },
\end{aligned}
$$

where $W_{\mu \nu}=D_{\mu} W_{\nu}-D_{\nu} W_{\mu}$ is the $W$ field strength using electromagnetic covariant derivatives, $D_{\mu}$. The coupling coefficients, $h_{L}$ and $h_{R}$ are given by

$$
\begin{aligned}
& h_{L}^{i j}=\delta \tilde{h}_{L}^{i j}+\tilde{V}^{i j}\left(1-\frac{\alpha S}{4\left(c_{w}^{2}-s_{w}^{2}\right)}+\frac{c_{w}^{2} \alpha T}{2\left(c_{w}^{2}-s_{w}^{2}\right)}+\frac{\alpha U}{8 s_{w}^{2}}-\frac{c_{w}^{2}\left(\Delta_{e}+\Delta_{\mu}\right)}{2\left(c_{w}^{2}-s_{w}^{2}\right)}\right), \\
& h_{R}^{i j}=\delta \tilde{h}_{R}^{i j},
\end{aligned}
$$

where $\tilde{V}^{i j}$ represents the unitary CKM matrices for the left-handed charged current interactions of the quarks $\left(\tilde{V}^{i j}=\tilde{V}_{q}^{i j}\right)$ and leptons $\left(\tilde{V}^{i j}=\tilde{V}_{\ell}^{i j}\right)$, assuming massive neutrinos. The coefficients $\delta \tilde{h}_{L(R)}^{u_{i} d_{j}}$ and $c_{L(R)}^{i j}$ represent a set of arbitrary nonstandard fermion- $W$ couplings. The parameters $S, T$ and $U$ are the usual 'oblique' corrections to the gauge boson vacuum polarizations as discussed above. Finally, $\Delta_{f}($ with $f=e, \mu$ or $\tau)$ denotes the quantity $\Delta_{f} \equiv \sqrt{\sum_{i}\left|h_{L}^{\nu_{i} f}\right|^{2}}-1$.

3. Neutral-Current Couplings: The general interactions between the light fermions and the $Z$ boson can be written as

$$
\mathcal{L}_{\mathrm{nc}}=-\frac{e}{s_{w} c_{w}}\left[\bar{f}_{i} \gamma^{\mu}\left(g_{L}^{i j} \gamma_{L}+g_{R}^{i j} \gamma_{R}\right) f_{j} Z_{\mu}+\bar{f}_{i} \sigma^{\mu \nu}\left(n_{L}^{i j} \gamma_{L}+n_{R}^{i j} \gamma_{R}\right) f_{j} Z_{\mu \nu}\right]
$$


where

$$
\begin{aligned}
g_{L(R)}^{i j}= & \delta \tilde{g}_{L(R)}^{i j}+\left(g_{L, R}^{i j}\right)_{S M}\left[1+\frac{1}{2}\left(\alpha T-\Delta_{e}-\Delta_{\mu}\right)\right] \\
& -Q_{i} \delta^{i j}\left(\frac{\alpha S}{4\left(c_{w}^{2}-s_{w}^{2}\right)}-\frac{c_{w}^{2} s_{w}^{2} \alpha T}{c_{w}^{2}-s_{w}^{2}}+\frac{c_{w}^{2} s_{w}^{2}\left(\Delta_{e}+\Delta_{\mu}\right)}{c_{w}^{2}-s_{w}^{2}}\right) .
\end{aligned}
$$

The SM couplings are $\left(g_{L}^{i j}\right)_{S M}=\left(T_{3 i}-Q_{i} s_{w}^{2}\right) \delta^{i j}$ and $\left(g_{R}^{i j}\right)_{S M}=\left(-Q_{i} s_{w}^{2}\right) \delta^{i j} \cdot Q_{i}$ is the electric charge of fermion $f_{i}$, and $T_{3 i}$ is its eigenvalue for the third component of weak isospin. $Z_{\mu \nu}$ is the abelian curl: $\partial_{\mu} Z_{\nu}-\partial_{\nu} Z_{\mu}$. The effective coupling matrices $\delta \tilde{g}_{L(R)}^{i j}$ and $n_{L(R)}^{i j}$ are arbitrary sets of nonstandard couplings between the fermions and the $Z$ boson.

4. The $W$ Mass: Besides the direct changes to the couplings between fermions and electroweak bosons, the low-energy Lagrangian also produces a deviation from the SM prediction for $M_{W}$ in terms of the three inputs, $M_{Z}, G_{F}$ and $\alpha$ 87]

$$
M_{W}^{2}=\left(M_{W}^{2}\right)_{S M}\left[1-\frac{\alpha S}{2\left(c_{w}^{2}-s_{w}^{2}\right)}+\frac{c_{w}^{2} \alpha T}{c_{w}^{2}-s_{w}^{2}}+\frac{\alpha U}{4 s_{w}^{2}}-\frac{s_{w}^{2}\left(\Delta_{e}+\Delta_{\mu}\right)}{c_{w}^{2}-s_{w}^{2}}\right] .
$$

We briefly note that an alternative, and widely used, parameterization for four of the parameters which appear in the above effective interactions is given by 82,88

$$
\delta \epsilon_{1}=\alpha T, \quad \delta \epsilon_{2}=-\frac{\alpha U}{4 s_{w}^{2}}, \quad \delta \epsilon_{3}=\frac{\alpha S}{4 s_{w}^{2}}, \quad \delta \epsilon_{b}=-2 \delta \tilde{g}_{L}^{b b} .
$$

The $\delta$ here indicates the deviation of these parameters from their SM values, computed using reference values, $\hat{m}_{t}$ and $\hat{m}_{H}$, for the top-quark and Higgs-boson masses. Of these two parameters, it is $m_{t}$ which is most important to follow because of the relatively strong dependence on it of low-energy observables. This dependence can be computed by determining the contributions of virtual top quarks to the various effective couplings. The dominant part of the result is proportional to $G_{F} m_{t}^{2}$, and is explicitly given by [20, 88, 89]:

$$
\begin{aligned}
\delta T_{S M} & \simeq \frac{3}{16 \pi s_{w}^{2} c_{w}^{2}}\left(\frac{m_{t}^{2}-\hat{m}_{t}^{2}}{M_{Z}^{2}}\right), \\
\left(\delta \tilde{g}_{L}^{b b}\right)_{S M} & \simeq \frac{\alpha}{16 \pi s_{w}^{2} c_{w}^{2}}\left(\frac{m_{t}^{2}-\hat{m}_{t}^{2}}{M_{Z}^{2}}\right) .
\end{aligned}
$$

The subdominant dependence on $m_{t}$ and $m_{H}$ has also been calculated, and explicit formulae may be found in the references. 


\subsection{Comparing to Experiment}

Given these effective interactions it is straightforward to compute their implications for experiments. The results to linear order in the small nonstandard effective couplings which describe the deviations from the Standard Model are given for a large number of low- and high-energy precision electroweak observables in Ref. [87. These expressions may then be fit to the data.

Not all of the possible effective interactions need be included in such a fit. For instance, many interactions cannot interfere with Standard Model contributions, and so cannot contribute to any observables to linear order in the new physics couplings. Among the interactions which do not appear for this reason are most of the flavor-changing interactions. Many of these can be independently constrained using experimental limits on flavor-changing processes, and Ref. [87] summarizes the resulting bounds.

The results of a fit of the general Lagrangian to the data, taking $\hat{m}_{t}=150$ $\mathrm{GeV}$ and $\hat{m}_{H}=300 \mathrm{GeV}$, are summarized in Tables 5, 6, and 7. Two types of fits are presented in these tables. For the 'Individual Fit' the parameter in question has been considered in isolation, with all of the other parameters set to zero by hand. This kind of fit is not realistic, but has often been considered in the literature. The 'Global Fit', on the other hand, allows all of the parameters to be floated while fitting the data. Perhaps surprisingly, the resulting bounds on the various parameters are nevertheless reasonably good, expressing the general success of the SM description. Since this success has been somewhat undermined in the most recent LEP results [24] - most notably in the branching ratio $R_{b}=$ $\Gamma(Z \rightarrow \bar{b} b) / \Gamma(Z \rightarrow$ hadrons $)$ - these fits are currently being updated to include this more recent data.

One of the applications of the bounds given in Tables 5 is to constrain the parameters of a specific model for nonstandard physics. The logic of such a constraint goes as follows. One first computes the effective Lagrangian which is obtained when all of the undiscovered (and assumed heavy) particles are integrated out. This results in a series of expressions for the effective couplings as functions of the couplings and masses of the underlying model. Next, the bounds from Tables 5 through 7 are used to constrain the parameters of the underlying model. The resulting bounds are generally weaker than those that would have been obtained if the model were fit directly to the experiments, since the fit whose results are described in these Tables permits all of the effective couplings to vary independently. Direct comparison of the results obtained in these two ways [87], shows that the bounds obtained often do not differ by much. This is typically because only a few experiments are responsible for the strongest experimental limits. 


\begin{tabular}{|c|c|c|}
\hline \hline Parameter & Individual Fit & Global Fit \\
\hline$S$ & $-0.10 \pm 0.16$ & $-0.2 \pm 1.0$ \\
$T$ & $+0.01 \pm 0.17$ & $-0.02 \pm 0.89$ \\
$U$ & $-0.14 \pm 0.63$ & $+0.3 \pm 1.2$ \\
\hline \hline
\end{tabular}

Table 5: Oblique Parameters. Results for the oblique parameters $S, T$ and $U$ obtained from the fit of the new-physics parameters to the data. The second column gives the result for the (unrealistic) case where all other parameters are constrained to vanish. Column three gives the result of a global fit in which all of the parameters of the effective Lagrangian are varied.

\begin{tabular}{|c|c|c|}
\hline \hline Parameter & Individual Fit & Global Fit \\
\hline$\Delta_{e}$ & $-0.0008 \pm .0010$ & $-0.0011 \pm .0041$ \\
$\Delta_{\mu}$ & $+0.00047 \pm .00056$ & $+0.0005 \pm .0039$ \\
$\Delta_{\tau}$ & $-0.018 \pm 0.008$ & $-0.018 \pm .009$ \\
$\mathcal{R} e\left(\delta \tilde{h}_{L}^{u d}\right)$ & $-0.00041 \pm .00072$ & $+0.0001 \pm .0060$ \\
$\mathcal{R} e\left(\delta \tilde{h}_{R}^{u d}\right)$ & $-0.00055 \pm .00066$ & $+0.0003 \pm .0073$ \\
$\mathcal{I} m\left(\delta \tilde{h}_{R}^{u d}\right)$ & $0 \pm 0.0036$ & $-0.0036 \pm .0080$ \\
$\mathcal{R} e\left(\delta \tilde{h}_{L}^{u s}\right)$ & $-0.0018 \pm .0032$ & - \\
$\mathcal{R} e\left(\delta \tilde{h}_{R}^{u s}\right)$ & $-0.00088 \pm .00079$ & $+0.0007 \pm .0016$ \\
$\mathcal{I} m\left(\delta \tilde{h}_{R}^{u s}\right)$ & $0 \pm 0.0008$ & $-0.0004 \pm .0016$ \\
$\mathcal{R e}\left(\delta \tilde{h}_{L}^{u b}\right), \mathcal{I}_{m}\left(\delta \tilde{h}_{L}^{u b}\right)$ & $-0.09 \pm .16$ & - \\
$\Sigma_{1}$ & - & $+0.005 \pm .027$ \\
$\mathcal{R} e\left(\delta \tilde{h}_{R}^{u b}\right)$ & - & - \\
$\mathcal{R} e\left(\delta \tilde{h}_{L}^{c d}\right)$ & $+0.11 \pm .98$ & - \\
$\operatorname{Re}\left(\delta \tilde{h}_{R}^{c d}\right)$ & - & - \\
$\operatorname{Re}\left(\delta \tilde{h}_{L}^{c s}\right)$ & $+0.022 \pm .20$ & - \\
$\operatorname{Re}\left(\delta \tilde{h}_{R}^{c s}\right)$ & $+0.022 \pm .20$ & - \\
$\operatorname{Re}\left(\delta \tilde{h}_{L}^{c b}\right)$ & $+0.5 \pm 4.6$ & - \\
$\Sigma_{2}$ & - & - \\
$\operatorname{Re}\left(\delta \tilde{h}_{R}^{c b}\right)$ & - & - \\
\hline \hline
\end{tabular}

Table 6: Charged-Current Parameters. More results of the fits of the new-physics parameters to the data. The quantities $\Sigma_{1}$ and $\Sigma_{2}$ arise in tests for the unitarity of the CKM matrix, and are defined as: $\Sigma_{1} \equiv \operatorname{Re} e\left(\delta \tilde{h}_{L}^{u s}\right)+\left[\mathcal{R} e\left(V_{u b}\right) \operatorname{Re}\left(\delta \tilde{h}_{L}^{u b}\right)+\operatorname{Im}_{m}\left(V_{u b}\right) \operatorname{Im}_{m}\left(\delta \tilde{h}_{L}^{u b}\right)\right] /\left|V_{u s}\right|$ and $\Sigma_{2} \equiv \operatorname{Re}\left(\delta \tilde{h}_{L}^{c d}\right)+\left|V_{c s}\right| \mathcal{R e}\left(\delta \tilde{h}_{L}^{c s}+\delta \tilde{h}_{R}^{c s}\right) /\left|V_{c d}\right|+\left|V_{c b}\right| \mathcal{R e}\left(\delta \tilde{h}_{L}^{c b}\right) /\left|V_{c d}\right|$. Blanks indicate where the corresponding fit would be inappropriate, such as for when a parameter always appears in a particular combination with others, and so cannot be individually fit. 


\begin{tabular}{|c|c|c|}
\hline \hline Parameter & Individual Fit & Global Fit \\
\hline$\delta \tilde{g}_{L}^{d d}$ & $+0.0016 \pm .0015$ & $+0.003 \pm .012$ \\
$\delta \tilde{g}_{R}^{d d}$ & $+0.0037 \pm .0038$ & $+0.007 \pm .015$ \\
$\delta \tilde{g}_{L}^{u u}$ & $-0.0003 \pm .0018$ & $-0.002 \pm 0.014$ \\
$\delta \tilde{g}_{R}^{u u}$ & $+0.0032 \pm .0032$ & $-0.003 \pm .010$ \\
$\delta \tilde{g}_{L}^{s s}$ & $-0.0009 \pm .0017$ & $-0.003 \pm .015$ \\
$\delta \tilde{g}_{R}^{s s}$ & $-0.0052 \pm .00095$ & $+0.002 \pm .085$ \\
$\delta \tilde{g}_{L}^{c c}$ & $-0.0011 \pm .0021$ & $+0.001 \pm .018$ \\
$\delta \tilde{g}_{R}^{c c}$ & $+0.0028 \pm .0047$ & $+0.009 \pm .029$ \\
$\delta \tilde{g}_{L}^{b b}$ & $-0.0005 \pm .0016$ & $-0.0015 \pm .0094$ \\
$\delta \tilde{g}_{R}^{b b}$ & $+0.0019 \pm .0083$ & $0.013 \pm .054$ \\
$\delta \tilde{g}_{L}^{\nu_{e} \nu_{e}}$ & $-0.0048 \pm .0052$ & - \\
$\delta \tilde{g}_{L}^{\nu_{\mu} \nu_{\mu}}$ & $-0.0021 \pm .0027$ & $+0.0023 \pm .0097$ \\
$\delta \tilde{g}_{L}^{\nu_{\tau} \nu_{\tau}}$ & $-0.0048 \pm .0052$ & - \\
$\delta \tilde{g}_{L}^{\nu_{e} \nu_{e}}+\delta \tilde{g}_{L}^{\nu_{\tau} \nu_{\tau}}$ & - & $-0.004 \pm .033$ \\
$\delta \tilde{g}_{L}^{e e}$ & $-0.00029 \pm .00043$ & $-0.0001 \pm .0032$ \\
$\delta \tilde{g}_{R}^{e e}$ & $-0.00014 \pm .00050$ & $+0.0001 \pm .0030$ \\
$\delta \tilde{g}_{L}^{\mu \mu}$ & $+0.0040 \pm .0051$ & $+0.005 \pm .032$ \\
$\delta \tilde{g}_{R}^{\mu \mu}$ & $-0.0003 \pm .0047$ & $+0.001 \pm .028$ \\
$\delta \tilde{g}_{L}^{\tau \tau}$ & $-0.0021 \pm .0032$ & $0.000 \pm .022$ \\
$\delta \tilde{g}_{R}^{\tau \tau}$ & $-0.0034 \pm .0028$ & $-0.0015 \pm .019$ \\
\hline \hline
\end{tabular}

Table 7: Neutral-Current Parameters. More results of the fits of the new-physics parameters to the data. As before, blanks indicate where the corresponding fit would be inappropriate, such as for when a parameter always appears in a particular combination with others, and so cannot be individually fit. 


\section{$5 \quad g-2$ of the Muon}

Magnetic moments of elementary particles receive radiative contributions which can in principle be sensitive to new degrees of freedom and interactions. The combination of larger mass and relatively long lifetime of the muon allows measurements of its anomalous magnetic moment, $a_{\mu} \equiv \frac{1}{2}(g-2)$, which are sensitive to large energy scales and very high order radiative corrections. The current experimental value of $a_{\mu}^{\exp }=116592300(840) \times 10^{-11}$ [90] is in good agreement with the theoretical calculation of $a_{\mu}^{\text {th }}=116591877(176) \times 10^{-11}$ 91, where the numbers in parentheses are the uncertainties. Agreement at this level includes QED corrections to $\mathcal{O}\left(\alpha^{5}\right)$ and hadronic vacuum polarization to $\mathcal{O}\left(\alpha^{3}\right)$. The Brookhaven E821 muon $g-2$ experiment is expected to reduce the experimental error in $a_{\mu}^{\exp }$ to below $\pm 40 \times 10^{-11}[92$. At this level of precision, electroweak radiative corrections are important and new physics at the weak scale can be probed.

In order to exploit the experimental precision of the Brookhaven experiment as a probe for new physics it is necessary to understand the SM contributions. These are usually given as $a_{\mu}=a_{\mu}^{\mathrm{QED}}+a_{\mu}^{\mathrm{had}}+a_{\mu}^{E W}$. The QED contributions have been calculated to $\mathcal{O}\left(\alpha^{5}\right)$, including the $\tau$ vacuum polarization contributions, and give $a_{\mu}^{\mathrm{QED}}=116584708(5) \times 10^{-11}$ [93. The hadronic contributions are of two types. The first corresponds to effects which represent the contribution of running $\alpha$ from low to high scales. These can not be calculated from first principles, but can be related to $R(s)=\sigma\left(e^{+} e^{-} \rightarrow\right.$ hadrons $) / \sigma\left(e^{+} e^{-} \rightarrow \mu^{+} \mu^{-}\right)$by means of a dispersion relation. A recent evaluation at $\mathcal{O}\left(\alpha^{2}\right)$ of the available data gives $a_{\mu}^{\text {had-vac-pol }}=7024(153) \times 10^{-11}$ 94]. The $\mathcal{O}\left(\alpha^{3}\right)$ corrections to the hadronic vacuum polarization have been calculated to be $a_{\mu}^{\text {had-vac-pol' }}=-90(5) \times 10^{-11} 91$. The other type of hadronic contributions are from light by light hadron amplitudes. Unfortunately these can not be related to other experimental observables but they can be estimated within some theoretical model. Recent estimates in a $1 / N_{c}$ expansion of a Nambu-Jona-Lasinio model give $a_{\mu}^{\text {had }-\gamma-\gamma}=8(9) \times 10^{-11}$ [95. The electroweak contributions which arise at one-loop from integrating out the $W$ and $Z$ bosons are $a_{\mu}^{\mathrm{EW}-1-\mathrm{loop}}=195 \times 10^{-11}$, which is roughly 5 times the expected experimental precision of the Brookhaven experiment. Because of the large number of diagrams, the two-loop electroweak contributions are not insignificant. A complete calculation of the two-loop fermionic and partial bosonic contributions gives $a_{\mu}^{\mathrm{EW}-2-\text { loop }}=-43(3) \times 10^{-11}$ 96. Of all the SM contributions, the hadronic vacuum polarization is by far the most uncertain. In order to test the one-loop electroweak corrections, the uncertainty in $R(s)$ at hadronic energies must be reduced by roughly a factor of four. Ongoing experiments at VEPP-2M together with future experiments at DA $\Phi$ NE and BEPC will hopefully reach this level.

Beyond the electroweak corrections, new degrees of freedom or interactions at the weak scale can in principle give important contributions to $a_{\mu}$. The anomalous 
magnetic moment operator

$$
\mathcal{L}=-a_{\mu} \frac{1}{4 m_{\mu}} \bar{\psi} \sigma_{\nu \rho} \psi F^{\nu \rho}
$$

is chirality violating, and must therefore vanish with the muon mass. This operator is therefore effectively dimension-six, being suppressed by two powers of the scale $M$ characterizing the new physics, $a_{\mu}^{\text {new }} \propto m_{\mu}^{2} / M^{2}$. The magnitude of $a_{\mu}^{\text {new }}$ is sensitive to the specific form of the new physics. In strongly coupled theories such as composite or technicolor models, contributions to $a_{\mu}^{\text {new }}$ can arise which are suppressed only by the scale $M$, i.e., $a_{\mu}^{\text {new }} \sim e m_{\mu}^{2} / M^{2}$ [97]. In this case the expected precision of the Brookhaven experiment would be sensitive at the $2 \sigma$ level to physics up to a scale $M \sim 2 \mathrm{TeV}$. The electroweak loop corrections are also sensitive to possible composite structures of the gauge bosons or gauge couplings. For example, the Brookhaven experiment will be sensitive to the anomalous moment of the $W^{ \pm}$ boson, $e(\kappa-1) F^{\mu \nu} W_{\mu}^{+} W_{\nu}^{-}$, at the $2 \sigma$ level of $|\kappa-1|>.07$ for $M \sim 1 \mathrm{TeV}$ 97.

For weakly coupled extensions of the SM the contributions to $a_{\mu}^{\text {new }}$ arise from radiative corrections and are suppressed by a perturbative loop factor of $a_{\mu}^{\text {new }} \sim$ $e(\alpha / 4 \pi) m_{\mu}^{2} / M^{2}$. As an example, supersymmetric extensions of the SM can give contributions that are generally the same order as the electroweak contributions 98. Imposing universality and radiative electroweak symmetry breaking, one finds that chargino-sneutrino loops typically dominate the supersymmetric contribution 99. Large values of $\tan \beta$ enhance the coupling of the Higgsino component of the chargino to the muon, and therefore maximize these types of contributions. The sign of $a_{\mu}^{\text {SUSY }}$ turns out to be correlated with that of the $\mu$ parameter in this region of parameter space. Assuming $a_{\mu}^{\exp }=a_{\mu}^{\mathrm{SM}}+a_{\mu}^{\mathrm{SUSY}}$, and that the Brookhaven experiment would be consistent with $a_{\mu}^{\mathrm{SM}}$ at the $2 \sigma$ level, it would then force the universal scalar mass and gluino mass (assuming gaugino universality) to be $\lesssim 500-600 \mathrm{GeV}$ for $\tan \beta=30$ 99. The precise bound will depend on how well $a_{\mu}^{\text {had }}$ can be determined from $R(s)$.

Models in which the muon mass has a sizeable (or sole) component arising from radiative corrections can give interesting contributions to $a_{\mu}^{\text {new }}$. In these types of models the muon (and perhaps other light SM fermions) is protected at tree level from obtaining a mass by some approximate chiral symmetry. The chiral symmetry is not respected radiatively, and the muon obtains a mass for example at one-loop, $m_{\mu} \sim\left(\lambda^{2} / 16 \pi^{2}\right) m_{\chi}$, where $\lambda$ is a Yukawa coupling, and $m_{\chi}$ is a parameter characterizing the breaking of the chiral symmetry (such as the mass of another fermion). A contribution to $a_{\mu}^{\text {new }}$ is generated at the same order as $m_{\mu}$, giving $a_{\mu}^{\text {new }} \sim e m_{\mu}^{2} / M^{2}$. Notice that this is not suppressed by a loop factor even though it arises perturbatively. This is because both the mass and anomalous magnetic moment arise perturbatively. If the muon mass is (largely) generated radiatively at or just above the weak scale, "sizeable" deviations of $a_{\mu}$ from the SM model prediction can therefore result. As an example, in supersymmetric 
theories with flavor violation in the slepton sector, the muon can receive a radiative contribution from the tau, $\delta m_{\mu} \sim\left(\sin \theta_{\mu \tau}\right)^{2}(\alpha / 4 \pi) m_{\tau}$ where $\sin \theta_{\mu \tau}$ is a slepton mixing angle. The supersymmetric contributions to $a_{\mu}$ could then be a factor $\sim$ $\left(\sin \theta_{\mu \tau}\right)^{2}\left(m_{\tau} / m_{\mu}\right)$ larger than the supersymmetric contributions mentioned above.

\section{Rare Processes in the Quark Sector}

We next investigate the indirect effects of new physics in processes which are rare or forbidden in the SM. In this Section we turn our attention to the quark sector, examining each quark flavor separately.

\subsection{Kaons}

Numerous processes involving Kaons occur through CP violation or flavor-changing neutral currents. As these two effects are small in the SM, Kaons provide a fruitful testing ground for virtual effects of new physics. For this reason, rare Kaon processes have played a strong and historical role in constraining new interactions. For example, the strongest bound (albeit assumption dependent) on the mass of a right-handed $W$ boson in the LRM is derived from its contribution to $K^{0}-\bar{K}^{0}$ mixing [100], the requirement of near degeneracy of squark masses results from a super-GIM mechanism imposed in the $K$ sector [101], and $K^{0}-\bar{K}^{0}$ mixing and $K_{L} \rightarrow \mu e$ have provided severe constraints on technicolor model building [102] forcing the introduction of a Techni-GIM mechanism. The observation of FCNC in the mass difference of neutral Kaons as well as the first observation of indirect $\mathrm{CP}$ violation (parameter $\epsilon_{K}$ ) provide tight constraints on several other models of new physics. Once these are taken into account and combined with the constraints from $B-\bar{B}$ mixing (and $b \rightarrow s \gamma$ ) the range of predictions for $\mathrm{K}$ decays are severely restricted. Nevertheless several Kaon processes remain sensitive to FCNC generated by new interactions at tree or loop level as well as to new mechanisms of $\mathrm{CP}$ violation.

Considering the difficulties encountered with model independent analyses of new physics in Kaon processes due to the large number of parameters involved, only typical models will be discussed here. These include the MSSM, the LRM, 3HDM, models with light leptoquarks or family symmetries, as well as non-minimal SUSY models; all of which are described in Section 2. For the discussion, we will concentrate on the processes with the least theoretical uncertainties and will specifically emphasize the models that could lead to enhancements of the SM prediction as experiments are expected, at best, to reach the SM level (as demonstrated in Table 8). 


\begin{tabular}{|c|c|c|c|}
\hline Observable & $\begin{array}{c}\text { Standard } \\
\text { Model }\end{array}$ & $\begin{array}{l}\text { Present Limit } \\
\text { (Exp.) }\end{array}$ & $\begin{array}{l}\text { Expected (Exp.) } \\
\text { Sensitivity }\end{array}$ \\
\hline$\epsilon^{\prime} / \epsilon$ & $(1-15) \times 10^{-4}$ & $\begin{array}{c}(23 \pm 7) \times 10^{-4}(\mathrm{NA} 31) \\
(7.4 \pm 5.9) \times 10^{-4}(\mathrm{E} 731)\end{array}$ & $\begin{array}{c}10^{-4} \text { (CERN-NA31) } \\
10^{-4} \text { (FNAL-E731) } \\
10^{-4}(\mathrm{DA} \Phi N E)\end{array}$ \\
\hline$K_{L} \rightarrow \mu^{ \pm} e^{\mp}$ & 0 & $3.3 \times 10^{-11}($ AGS-791) & $10^{-12}$ (BNL-E871) \\
\hline$K^{+} \rightarrow \pi^{+} \mu^{ \pm} e^{\mp}$ & 0 & $2.1 \times 10^{-10}(\mathrm{AGS})$ & $10^{-12}$ (BNL-E865) \\
\hline$K^{+} \rightarrow \pi^{+} \nu \bar{\nu}$ & $(.7-3) \times 10^{-10}$ & $5.2 \times 10^{-9}($ AGS-787) & $10^{-10}$ (BNL-E787) \\
\hline$K_{L} \rightarrow \pi^{0} \nu \bar{\nu}$ & $.2-8 \times 10^{-11}$ & $2.2 \times 10^{-4}($ FNAL $) 731$ & $10^{-8}$ (FNAL-799) \\
\hline$K_{L} \rightarrow \pi^{0} e^{+} e^{-}$ & $.1-2 \times 10^{-11}$ & $3.2 \times 10^{-7}(\mathrm{BNL}$ E780 $)$ & 10-11 (FNAL-799II) \\
\hline$P_{T}\left(K^{+} \rightarrow \pi^{0} \mu \nu\right)$ & $10^{-6}$ & $-3.1 \pm 5.3 \times 10^{-3}(\mathrm{BNL})$ & $5 \times 10^{-4}(\mathrm{KEK}-\mathrm{E} 246)$ \\
\hline
\end{tabular}

Table 8: Present limit and future prospects for Kaon processes[103,104].

\subsubsection{Direct CP violation, $\epsilon^{\prime} / \epsilon$}

The importance of this measurement to understand more about the mechanism of $\mathrm{CP}$ violation cannot be overemphasized, although, due to conflicting experimental results, constraints on new physics from $\epsilon^{\prime} / \epsilon$ will not be taken into account here. The next round of experiments, which will reach a precision of $10^{-4}$, might settle the issue of whether or not $\epsilon^{\prime} / \epsilon \neq 0$. The SM predicts a non-zero value but allows for a wide range (see Table 8). Ultimately one wants to establish whether $\mathrm{CP}$ violation is milliweak $(\Delta S=1)$ as in the $\mathrm{SM}$ and/or superweak $(\Delta S=2)$. The latter occurs in multi-Higgs doublet models through scalar interactions, in SUSY models[105], or in the LRM to give a few examples[106]. While beyond the standard models often meet difficulties in reproducing the value of $\epsilon$, this can be circumvented by allowing for the standard source of $\mathrm{CP}$ violation in addition to the new superweak interaction. A summary of potential effects of new physics in a chosen set of Kaon processes are given in Table 9 .

\subsubsection{Lepton-number violation}

The most severe upper limits on rare $\mathrm{K}$ decays have been obtained for $K_{L} \rightarrow \mu^{ \pm} e^{\mp}$ and $K^{+} \rightarrow \pi^{+} \mu^{ \pm} e^{\mp}$. These decays feature a good sensitivity to new physics as they are strictly forbidden in the SM unless neutrinos have masses. Furthermore, the present limits on neutrino masses and mixings imply a decay rate which is orders of magnitude below the sensitivity of planned experiments 107. The high sensitivity to new interactions is best illustrated by a generic model independent bound on the scale of new physics that generates $K_{L} \rightarrow \mu^{ \pm} e^{\mp},(\Lambda>108(420) \mathrm{TeV})$ for purely left-handed (scalar) operators. The process $K^{+} \rightarrow \pi^{+} \mu^{ \pm} e^{\mp}$ while being less sensitive, probes a different set of operators; axial-vector or pseudoscalar operators 


\begin{tabular}{|c|c|c|c|c|c|c|}
\hline \hline & 3HDM & MSSM & LR & LQ & Hor. & Comments \\
\hline$\epsilon^{\prime} / \epsilon$ & $* *$ & - & $* *$ & - & $*$ & contributions from \\
& & & & & & non-minimal SUSY \\
$K_{L} \rightarrow \mu^{ \pm} e^{\mp}$ & - & - & - & $* *$ & $* *$ & \\
$K^{+} \rightarrow \pi^{+} \nu \bar{\nu}$ & $*$ & - & - & $* *$ & $* *$ & large in SUSY \\
& & & & & & with $R$ parity \\
$P_{T}\left(K^{+} \rightarrow \pi^{0} \mu \nu\right)$ & $* *$ & - & - & $* *$ & - & \\
$P_{L}\left(K_{L} \rightarrow \mu^{+} \mu^{-}\right)$ & - & - & $* *$ & $* *$ & - & \\
\hline \hline
\end{tabular}

Table 9: Effect of new physics in Kaon processes. A double(single) star indicates possibly strong (mild) enhancement over the SM while a dash stands for no measurable effect.

as opposed to the vector or scalar case 108.

These limits can be translated into mass bounds on the new particles that could generate these decays at tree-level. In general, new particles that can induce tree-level flavor changes, in particular vector bosons, will also contribute to $\Delta M_{K}$ (as well as to $K^{+} \rightarrow \pi^{+} \nu \bar{\nu}$ ). Unless some symmetry allows one to avoid the latter constraints, it is unlikely that a signal would be observed in $K_{L} \rightarrow \mu^{ \pm} e^{\mp}$. However, several models possess this type of symmetry, for example models with family symmetries. Leptoquark models naturally avoid the constraint as they do not contribute to $\Delta M_{K}$ [109] at tree-level. The same arguments apply also to cases where the lepton number violating decays are induced at the loop level. For example in the MSSM, the flavor change arises through mixing among the quark and lepton superpartners. These mixings are constrained both by $\Delta M_{K}$ and $\mu \rightarrow e \gamma$, so that the rate for $K_{L} \rightarrow \mu^{ \pm} e^{\mp}$ is expected to be at most $10^{-14}[109]$.

\subsubsection{Rare decays}

The standard model level for the theoretically clean decay, $K^{+} \rightarrow \pi^{+} \nu \bar{\nu}$ should be reached in the next decade. This transition is theoretically clean as it is short-distance dominated [110], the relevant hadronic operator is extracted from $K^{+} \rightarrow \pi^{0} e^{+} \nu$, and the next-to-leading order QCD corrections are fully known [111]. An enhancement over the SM rate would clearly signal new physics although such enhancements are not expected in most minimal extensions of the SM once the constraints from $B-\bar{B}$ and $\epsilon_{K}$ are taken into account [112]. These processes are to a large extent governed by the same parameters, limiting the impact of new physics in this case. A possible exception concerns the MSSM with SUSY particles in the $100 \mathrm{GeV}$ range where there can be some enhancement 113. There remains the possibility of large enhancements in SUSY models with broken R-parity, models with family symmetry producing a new type of neutrino, as well as certain lep- 
toquark models 112. Typically these models are more weakly constrained overall and could also lead to non standard signals in other rare processes (for example B or $\mathrm{D}$ decays). The $3 \mathrm{HDM}$ also can lead to a moderate enhancement (by a factor 3 ) of the standard rate for this decay [11]. This is to be contrasted with the 2HDM where the existing constraints preclude any significant effect in future kaon decays measurements [10].

The process $K_{L} \rightarrow \mu^{+} \mu^{-}$shares several features of the preceding one as far as sensitivity to new physics is concerned. However, the bounds obtained are not as reliable due to large and uncertain long distance contributions. One interesting aspect of this process is the sensitivity to other sources of CP violation in the measurement of the longitudinal polarization of the muon, which is expected to be $P_{L} \approx 2 \times 10^{-3}$ in the SM. Both SUSY models with leptoquarks and the LRM with neutrino mixing can produce large polarizations, while non-supersymmetric models with leptoquarks predict a small polarization 114.

Both processes $K_{L} \rightarrow \pi^{0} \nu \bar{\nu}, K_{L} \rightarrow \pi^{0} e^{+} e^{-}$are sensitive to new sources of $\mathrm{CP}$ violation [115], the former being basically free of long distance effect but representing a true challenge for experimentalists (to wit the present limit on this decay) while the second has larger theoretical uncertainties. Many models predict rates higher than the standard one and the hope to observe those decays rests on the presence of new physics. Leptoquarks for example can possibly lead to large enhancements 113.

\subsubsection{Transverse muon polarization in $K^{+} \rightarrow \pi^{0} \mu \nu$}

The T-violating, transverse polarization of the muon $\left(P_{T}\right)$ in $K^{+} \rightarrow \pi^{0} \mu \nu$ provides sensitive tests of models with new sources of $\mathrm{CP}$ violation. Indeed, in the SM, or in any model with only vector and/or axial-vector interactions, $P_{T}$ is expected to be very small; such is the case in the MSSM and the LRM. On the other hand, in multiple Higgs models where the $\mathrm{CP}$ or $\mathrm{T}$ violation arises in the scalar sector from a phase in the charged Higgs mixing matrix a polarization can be induced. It has the form $P_{T} \propto \operatorname{Im}\left(\alpha_{1} \beta_{1}^{*}\right)\left(m_{K} / m_{H^{+}}\right)^{2}\left(v_{2} / v_{3}\right)^{2}$ where $\alpha_{1}$ and $\beta_{1}$ are the Yukawa coupling of the charged Higgs to quarks (corresponding to $X_{1}$ and $Y_{1}$, respectively, in Eq. 2) and $v_{2} / v_{3}$ is the ratio of vev's. At present the rate for $B\left(B \rightarrow X \tau \nu_{\tau}\right)$ which depends on the same parameters gives the strongest constraint on the 3HDM (see Fig. 10). The planned order of magnitude improvement on the search for this polarization effect (see Table 8) should probe a large region of the remaining parameter space as illustrated in Fig. 10 from Ref. [104]. The Imaginary part of the Yukawa coupling in some otherwise unconstrained leptoquark models can also induced large polarization through tree-level leptoquark exchanges 116]. 
Figure 10: Constraints on the parameters of the $3 \mathrm{HDM}$ for $M_{H^{+}}=2 M_{Z}$ from (a)the neutron electric dipole moment; (b) $b \rightarrow s \gamma$; (c) $P_{T}\left(K^{+} \rightarrow \pi^{0} \mu \nu\right)$; (d) $b \rightarrow X \tau \nu$. The dotted line shows the expected sensitivity in $P_{T}$ at KEK-E246.

\subsubsection{Discussion}

From inspection of Table 9 one concludes that rare $\mathrm{K}$ decays are not very sensitive to indirect effects from the MSSM, although they can probe more general SUSY models. In general leptoquark models can potentially give strong signals in all rare decays and polarization measurements. This is simply a reflection of the fact that these models are poorly constrained at present. Other models could have measurable effects in only a few processes. As it is expected to be too small, the effect of an anomalous $W W \gamma$ coupling is not included. Indeed, the best limit obtained on the $\mathrm{C}$ and $\mathrm{P}$ violating coupling $g_{5}$ from the decays $K_{L} \rightarrow \mu^{+} \mu^{-}$and $K^{+} \rightarrow \pi^{+} \nu \bar{\nu}$ is of order 1 while we expect $g_{5}<10^{-2}$ [117].

\subsection{Charm-Quark Sector}

While investigations of the $K$ and $B$ systems have and will continue to play a central role in our quest to understand flavor physics, in-depth examinations of the charm-quark sector have yet to be performed, leaving a gap in our knowledge. Since charm is the only heavy charged $+2 / 3$ quark presently accessible to experiment in copious amounts, it provides the sole window of opportunity to examine flavor physics in this sector. In addition, charm allows a complimentary probe of SM physics (and beyond) to that attainable from the down-quark sector.

Due to the effectiveness of the GIM mechanism, short distance SM contribu- 


\begin{tabular}{|c|c|c|}
\hline \hline Meson & $\mu^{+} \nu_{\mu}$ & $\tau^{+} \nu_{\tau}$ \\
\hline$D^{+}$ & $3.52 \times 10^{-4}$ & $9.34 \times 10^{-4}$ \\
$D_{s}^{+}$ & $4.21 \times 10^{-3}$ & $4.11 \times 10^{-2}$ \\
\hline \hline
\end{tabular}

Table 10: SM branching fractions for the leptonic decay modes, assuming $f_{D}=200$ $\mathrm{MeV}$ and $f_{D_{s}}=230 \mathrm{MeV}$.

tions to rare charm processes are very small. Most reactions are thus dominated by long range effects which are difficult to reliably calculate. However, for some interactions, there exists a window for the potential observation of new physics. In fact, it is precisely because the SM flavor changing neutral current rates are so small that charm provides an untapped opportunity to discover new effects and offers a detailed test of the SM in the up-quark sector. Here, we will examine leptonic decays, rare decays, $D^{0}-\bar{D}^{0}$ mixing, and $\mathrm{CP}$ violation in the decays of charmed mesons.

\subsubsection{Leptonic Decays of Charmed Mesons}

The SM transition rate for the purely leptonic decay of a pseudoscalar charm meson is

$$
\Gamma\left(D_{(q)}^{+} \rightarrow \ell^{+} \nu_{\ell}\right)=\frac{G_{F}^{2}}{8 \pi} f_{D_{(q)}}\left|V_{c q}\right|^{2} m_{D_{(q)}} m_{\ell}^{2}\left(1-\frac{m_{\ell}^{2}}{m_{D_{(q)}}^{2}}\right)^{2},
$$

with $q=d, s$ and $f_{D_{(q)}}$ is the weak decay constant defined as usual by

$$
\left\langle 0\left|\bar{q} \gamma_{\mu} \gamma_{5} c\right| D_{(q)}(\mathbf{p})\right\rangle=i f_{D_{(q)}} p_{\mu}
$$

where $f_{\pi}=131 \mathrm{MeV}$ in this normalization. The resulting branching fractions are small due to the helicity suppression and are listed in Table 10 using the central values of the CKM parameters given in Ref. [5]. Assuming that the CKM matrix elements are well-known, the leptonic decays can provide important information on the value of the pseudoscalar decay constants. Precise measurements of these constants are essential for the study of $D^{0}-\bar{D}^{0}$ mixing, CP violation, and nonleptonic decays.

Non-SM contributions may affect these purely leptonic decays. Signatures for new physics include the measurement of non-SM values for the absolute branching ratios, or the observation of a deviation from the SM prediction for the ratio

$$
\frac{B\left(D_{(s)}^{+} \rightarrow \mu^{+} \nu_{\mu}\right)}{B\left(D_{(s)}^{+} \rightarrow \tau^{+} \nu_{\tau}\right)}=\frac{m_{\mu}^{2}\left(1-m_{\mu}^{2} / m_{D_{(s)}}^{2}\right)^{2}}{m_{\tau}^{2}\left(1-m_{\tau}^{2} / m_{D_{(s)}}^{2}\right)^{2}}
$$

This ratio is sensitive to violations of $\mu-\tau$ universality. As a specific example, we consider the case where the SM Higgs sector is enlarged by an additional Higgs 
doublet. These models generate important contributions 57 to the decay $B \rightarrow \tau \nu_{\tau}$ and it is instructive to examine their effects in the charm sector. In 2HDM Models I and II, we obtain

$$
B\left(D^{+} \rightarrow \ell^{+} \nu_{\ell}\right)=B_{\mathrm{SM}}\left(1+\frac{m_{D}^{2}}{m_{H^{ \pm}}^{2}}\right)^{2}
$$

where in Model II the $D_{s}^{+}$decay receives an additional modification

$$
B\left(D_{s}^{+} \rightarrow \ell^{+} \nu_{\ell}\right)=B_{\mathrm{SM}}\left[1+\frac{m_{D_{s}}^{2}}{m_{H^{ \pm}}^{2}}\left(1-\tan ^{2} \beta \frac{m_{s}}{m_{c}}\right)\right]^{2} .
$$

In this case, we see that the effect of the $H^{ \pm}$exchange is independent of the leptonic final state and the above prediction for the ratio in Eq. 57 is unchanged.

\subsubsection{Rare and Forbidden Decays of Charm Mesons}

FCNC decays of the $D$ meson include the processes $D^{0} \rightarrow \ell^{+} \ell^{-}, \gamma \gamma$, and $D \rightarrow$ $X_{u}+\gamma, X_{u}+\nu \bar{\nu}, X_{u}+\ell^{+} \ell^{-}$, with $\ell=e, \mu$. They proceed via electromagnetic or weak penguin diagrams as well as receiving contributions from box diagrams in some cases. The calculation of the SM short distance rates for these processes is straightforward and the transition amplitudes and standard loop integrals, which are categorized in Ref. 118 for rare $K$ decays, are easily converted to the $D$ system. The values of the resulting inclusive short distance branching fractions, before QCD corrections are applied, are shown in Table 11, along with the current experimental bounds [5, 119]. The leading order QCD corrections have recently been calculated 120 for the radiative decay and are found to greatly enhance the inclusive branching fraction giving $B\left(D \rightarrow X_{u} \gamma\right)=(4-8) \times 10^{-12}$. In all decay modes, the corresponding exclusive rates are typically an order of magnitude less than the inclusive case. We note that the transition $D^{0} \rightarrow \ell^{+} \ell^{-}$, is helicity suppressed and hence has the smallest branching fraction. The range given for this branching fraction, $(1-20) \times 10^{-19}$, indicates the effect of varying the parameters in the ranges $f_{D}=0.15-0.25 \mathrm{GeV}$ and $m_{s}=0.15-0.40 \mathrm{GeV}$.

The calculation of the long distance branching fractions are plagued with the usual hadronic uncertainties and the estimates listed in the table convey an upper limit on the size of these effects rather than an actual value. These estimates have been computed by considering various intermediate particle states (e.g., $\pi, K, \bar{K}, \eta, \eta^{\prime}, \pi \pi$, or $\left.K \bar{K}\right)$ and inserting the known rates for the decay of the intermediate particles into the final state of interest. In all cases we see that the long distance contributions overwhelm those from SM short distance physics, and hence would hide potential contributions from new physics. This is shown explicitly in Fig. 11, where the branching fraction $B\left(D \rightarrow X_{u} \gamma\right)$ is given in the four generation model as a function of the relevant fourth generation CKM mixing factor. We see 


\begin{tabular}{|c|c|c|c|}
\hline "Decay Mode & Experimental Limit & 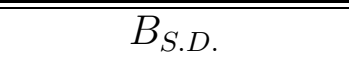 & $\overline{B_{L . D .} .}$ \\
\hline$D^{0} \rightarrow \mu^{+} \mu^{-}$ & $<3.3 \times 10^{-6}$ & $(1-20) \times 10^{-19}$ & $<3 \times 10^{-15}$ \\
\hline$D^{0} \rightarrow e^{+} e^{-}$ & $<1.3 \times 10^{-5}$ & $(2.3-4.7) \times 10^{-24}$ & \\
\hline$D^{0} \rightarrow \mu^{ \pm} e^{\mp}$ & $<1.9 \times 10^{-5}$ & 0 & 0 \\
\hline$D^{0} \rightarrow \gamma \gamma$ & - & $10^{-16}$ & $<3 \times 10^{-9}$ \\
\hline$D \rightarrow X_{u}+\gamma$ & & $1.4 \times 10^{-17}$ & \\
\hline$D^{0} \rightarrow \rho^{0} \gamma$ & $<1.4 \times 10^{-4}$ & & $<2 \times 10^{-5}$ \\
\hline$D^{0} \rightarrow \phi^{0} \gamma$ & $<2.0 \times 10^{-4}$ & & $<10^{-4}$ \\
\hline$D^{+} \rightarrow \rho^{+} \gamma$ & - & & $<2 \times 10^{-4}$ \\
\hline$D \rightarrow X_{u}+\ell^{+} \ell^{-}$ & & $4 \times 10^{-9}$ & \\
\hline$D^{0} \rightarrow \pi^{0} e e / \mu \mu$ & $<4.5 / 54 \times 10^{-5}$ & & \\
\hline$D^{0} \rightarrow \bar{K}^{0} e e / \mu \mu$ & $<1.1 / 6.7 \times 10^{-4}$ & & $<2 \times 10^{-15}$ \\
\hline$D^{0} \rightarrow \rho^{0} e e / \mu \mu$ & $<1.0 / 4.9 \times 10^{-4}$ & & \\
\hline$D^{+} \rightarrow \pi^{+} e e / \mu \mu$ & $<6.6 / 1.8 \times 10^{-5}$ & few $\times 10^{-10}$ & $<10^{-8}$ \\
\hline$D^{+} \rightarrow K^{+} e e / \mu \mu$ & $<480 / 8.5 \times 10^{-5}$ & & $<10^{-15}$ \\
\hline$D^{+} \rightarrow \rho^{+} \mu \mu$ & $<5.8 \times 10^{-4}$ & & \\
\hline$D^{0} \rightarrow X_{u}+\nu \bar{\nu}$ & & $2.0 \times 10^{-15}$ & \\
\hline$D^{0} \rightarrow \pi^{0} \nu \bar{\nu}$ & - & $4.9 \times 10^{-16}$ & $<6 \times 10^{-16}$ \\
\hline$D^{0} \rightarrow \bar{K}^{0} \nu \bar{\nu}$ & - & & $<10^{-12}$ \\
\hline$D^{+} \rightarrow X_{u}+\nu \bar{\nu}$ & - & $4.5 \times 10^{-15}$ & \\
\hline$D^{+} \rightarrow \pi^{+} \nu \bar{\nu}$ & - & $3.9 \times 10^{-16}$ & $<8 \times 10^{-16}$ \\
\hline$D^{+} \rightarrow K^{+} \nu \bar{\nu}$ & - & & $<10^{-14}$ \\
\hline
\end{tabular}

Table 11: Standard Model predictions for the branching fractions due to short and long distance contributions for various rare $D$ meson decays. Also shown are the current experimental limits $[5,119]$. 
Figure 11: Branching fraction for $D \rightarrow X_{u} \gamma$ in the four generation SM as a function of the appropriate CKM mixing factor, with the solid, dashed, dotted, dash-dotted curve corresponding to fourth generation quark masses $M_{b^{\prime}}=100,200,300,400 \mathrm{GeV}$, respectively.

that a sizable enhancement of the three generation rate is possible, however, the short distance rate is still overpowered by the long range effects.

Lepton flavor violating decays, e.g., $D^{0} \rightarrow \mu^{ \pm} e^{\mp}$ and $D \rightarrow X+\mu^{ \pm} e^{\mp}$, are strictly forbidden in the SM with massless neutrinos. In a model with massive non-degenerate neutrinos and non-vanishing neutrino mixings, such as in four generation models, $D^{0} \rightarrow \mu^{ \pm} e^{\mp}$ would be mediated by box diagrams with the massive neutrinos being exchanged internally. LEP data restricts 2] heavy neutrino mixing with $e$ and $\mu$ to be $\left|U_{N e} U_{N \mu}^{*}\right|^{2}<7 \times 10^{-6}$ for a neutrino with mass $m_{N}>45 \mathrm{GeV}$. Consistency with this bound constrains the branching fraction to be $B\left(D^{0} \rightarrow \mu^{ \pm} e^{\mp}\right)<6 \times 10^{-22}$. This same results also holds for a heavy singlet neutrino which is not accompanied by a charged lepton. The observation of this decay would be a clear signal for the existence of a different class of models with new physics. For example, leptoquarks can mediate $D^{0} \rightarrow \mu^{ \pm} e^{\mp}$ by treelevel exchange, although their contributions are suppressed by angular momentum conservation. From the present experimental bound on this process (as given in Table 11), Davidson et al. 18 derive the constraint on the leptoquark mass $m_{L Q}$ and coupling parameters (as defined in Section 2),

$$
\sqrt{F_{e u} F_{\mu c}}<4 \times 10^{-3} \frac{\alpha}{4 \pi}\left[\frac{m_{L Q}}{100 \mathrm{GeV}}\right]^{2}
$$




\subsection{3 $\quad D^{0}-\bar{D}^{0}$ Mixing and New Physics}

Currently, the best bound [5 on $D^{0}-\bar{D}^{0}$ mixing is from fixed target experiment, with $x_{D} \equiv \Delta m_{D} / \Gamma<0.083$ (where $\Delta m_{D}=m_{2}-m_{1}$ is the mass difference), yielding $\Delta m_{D}<1.3 \times 10^{-13} \mathrm{GeV}$. However, the data analysis in this case 121 was based on the assumption that there is no interference between the mixing signal and the dominant background of doubly Cabbibo suppressed decays. It has been recently noted [121] that while this assumption may be valid in the SM (since the expected size of mixing is small), it does not necessarily apply in models with new physics where $D^{0}-\bar{D}^{0}$ mixing is potentially large.

The short distance SM contributions to $\Delta m_{D}$ proceed through a $W$ box diagram with internal $d, s, b$-quarks. In this case the external momentum, which is of order $m_{c}$, is communicated to the light quarks in the loop and can not be neglected. The effective Hamiltonian is

$$
\mathcal{H}_{\text {eff }}^{\Delta c=2}=\frac{G_{F} \alpha}{8 \sqrt{2} \pi x_{w}}\left[\left|V_{c s} V_{u s}^{*}\right|^{2}\left(I_{1}^{s} \mathcal{O}-m_{c}^{2} I_{2}^{s} \mathcal{O}^{\prime}\right)+\left|V_{c b} V_{u b}^{*}\right|^{2}\left(I_{3}^{b} \mathcal{O}-m_{c}^{2} I_{4}^{b} \mathcal{O}^{\prime}\right)\right],
$$

where the $I_{j}^{q}$ represent integrals [122] that are functions of $m_{q}^{2} / M_{W}^{2}$ and $m_{q}^{2} / m_{c}^{2}$, and $\mathcal{O}=\left[\bar{u} \gamma_{\mu}\left(1-\gamma_{5}\right) c\right]^{2}$ is the usual mixing operator while $\mathcal{O}^{\prime}=\left[\bar{u}\left(1+\gamma_{5}\right) c\right]^{2}$ arises in the case of non-vanishing external momentum. The numerical value of the short distance contribution is $\Delta m_{D} \sim 5 \times 10^{-18} \mathrm{GeV}$ (taking $f_{D}=200 \mathrm{MeV}$ ). The long distance contributions have been computed via two different techniques: (i) the intermediate particle dispersive approach (using current data on the intermediate states) yields 123 $\Delta m_{D} \lesssim 10^{-16} \mathrm{GeV}$, and (ii) heavy quark effective theory which results [124 in $\Delta m_{D} \sim 10^{-17} \mathrm{GeV}$. Clearly, the SM predictions lie far below the present experimental sensitivity!

\section{- Fourth Generation Model}

One reason the SM short distance expectations for $D^{0}-\bar{D}^{0}$ mixing are so small is that there are no heavy particles participating in the box diagram to enhance the rate. Hence the first extension to the SM that we consider is the addition 125 of a heavy $Q=-1 / 3$ quark. We can now neglect the external momentum and $\Delta m_{D}$ is given by the usual expression [118],

$$
\Delta m_{D}=\frac{G_{F}^{2} M_{W}^{2} m_{D}}{6 \pi^{2}} f_{D}^{2} B_{D}\left|V_{c b^{\prime}} V_{u b^{\prime}}^{*}\right|^{2} F\left(m_{b^{\prime}}^{2} / M_{W}^{2}\right) .
$$

The value of $\Delta m_{D}$ is displayed in this model in Fig. 12(a) as a function of the overall CKM mixing factor for various values of the heavy quark mass. We see that $\Delta m_{D}$ approaches the current experimental range for large values of the mixing factor.

\section{- Multi-Higgs-Doublet Model}

Next we examine two-Higgs-doublet models discussed above which avoid treelevel FCNC by introducing a global symmetry. The expression for $\Delta m_{D}$ in these 


\begin{tabular}{|c|c|}
\hline \hline$(\mathrm{AB})$ & Upper Bound \\
\hline$(\mathrm{LL})^{2},(\mathrm{RR})^{2}$ & $(0.2)^{2}$ \\
$(\mathrm{LL})(\mathrm{RR})$ & $\left(3.6 \times 10^{-2}\right)^{2}$ \\
$(\mathrm{LR})^{2},(\mathrm{RL})^{2}$ & $\left(5.0 \times 10^{-2}\right)^{2}$ \\
$(\mathrm{LR})(\mathrm{RL})$ & $(0.1)^{2}$ \\
\hline \hline
\end{tabular}

Table 12: Bounds on $\delta \tilde{m}_{u_{A} c_{B}}^{2} / \tilde{m}_{0}^{2}$ from $\Delta m_{D}$, assuming a limit of $\Delta m_{D}<10^{-13} \mathrm{GeV}$.

models can be found in Ref. 18. From the Lagrangian in Eq. (2) it is clear that Model I will only modify the SM result for very small values of $\tan \beta$, and this region is already excluded 10, 126 from existing data on $b \rightarrow s \gamma$ and $B_{d}^{0}-\bar{B}_{d}^{0}$ mixing. However, enhancements can occur in Model II for large values of $\tan \beta$, as demonstrated in Fig. 12(b).

\section{- Supersymmetry}

Virtual exchange of squarks and gluinos in a SUSY-box diagram can have a strong contribution to $D^{0}-\bar{D}^{0}$ mixing. In this case mixing can be induced by flavor changing radiatively generated mass insertions. These are thought to be small in the MSSM, but can be large in non-minimal models 127. The resulting $\Delta C=2$ Hamiltonian is

$$
\begin{aligned}
\mathcal{H}_{S U S Y}^{\Delta c=2}=\frac{\alpha_{s}^{2}}{216 \tilde{m}_{0}} & {\left[\frac{\delta \tilde{m}_{u_{L} c_{L}}^{2}}{\tilde{m}_{0}^{2}} G\left(\frac{m_{\tilde{g}}^{2}}{\tilde{m}_{0}^{2}}\right)\left(\bar{u}_{L} \gamma_{\mu} c_{L}\right)\left(\bar{u}_{L} \gamma^{\mu} c_{L}\right)\right.} \\
+ & \left.(R R)^{2}+(L L)(R R)+(L R)^{2}+(L R)(R L)\right]
\end{aligned}
$$

where $\delta \tilde{m}_{u_{L} c_{L}}$ characterizes the mass insertion, the $\mathrm{G}(\mathrm{x})$ 's are known functions 105, and the remaining parameters are defined in Section 2. The experimental bound on $\Delta m_{D}$ can be translated into constraints on the various terms in the above Hamiltonian as shown in Table 12 .

\section{- Flavor Changing Neutral Higgs Model}

We now consider the case of extended Higgs sectors without natural flavor conservation. In this case, the lightest neutral higgs $h^{0}$ can now contribute to $\Delta m_{D}$ through tree-level exchange as well as mediating $D^{0}-\bar{D}^{0}$ mixing by $h^{0}$ and t-quark virtual exchange in a box diagram. These latter contributions only compete with those from the tree-level process for large values of $\Delta_{i j}$ (where $\Delta_{i j}$ is defined in Section 2). In Fig. 12(c-d) we show the value of $\Delta m_{D}$ in this model from these two types of contributions.

\section{- Leptoquark Models}

Leptoquarks participate in $\Delta m_{D}$ via virtual exchange inside a box diagram 18 , 
Figure 12: $\Delta m_{D}$ in (a) the four generation SM with the same labeling as in Fig. 11, (b) in two-Higgs-doublet model II as a function of $\tan \beta$ with, from top to bottom, the solid, dashed, dotted, dash-dotted, solid curve representing $m_{H^{ \pm}}=50,100,250,500,1000$ $\mathrm{GeV}$. The solid horizontal line corresponds to the present experimental limit. (c) Treelevel and (d) box diagrams contributions to $\Delta m_{D}$ in the flavor changing Higgs model described in the text as a function of the mixing factor for $m_{h}=50,100,250,500,1000$ $\mathrm{GeV}$ corresponding to the solid, dashed, dotted, dash-dotted, and solid curves from top to bottom. (e) Constraints in the leptoquark coupling-mass plane from $\Delta m_{D}$. (f) Values of $\Delta m_{D}$ in the Alternate Left-Right Symmetric Model as a function of the ratio of masses of the exotic fermion $h_{R}$ to the right-handed $W$. The curves represent the generational mass ratios for $h_{R}$ of $0.1,0.3,0.5,0.7$, and 0.9 , from top to bottom. 
together with a charged lepton or neutrino. Assuming that there is no leptoquarkGIM mechanism, and taking both exchanged leptons to be the same type, we obtain the restriction

$$
\frac{F_{\ell c} F_{\ell u}}{m_{L Q}^{2}}<\frac{196 \Delta m_{D}}{\left(4 \alpha f_{D}\right)^{2} m_{D}}
$$

where $F_{\ell q}$ is defined in Section 2. The resulting constraints in the leptoquark coupling-mass plane are presented in Fig. 12(e), assuming that a limit of $\Delta m_{D}<$ $10^{-13} \mathrm{GeV}$ could be obtained from experiment.

\section{- Alternate Left-Right Symmetric Model}

As discussed in Section 2, in this model the right-handed $W$ boson couples the right-handed up-quarks to the exotic charged $-1 / 3 h_{R}$ fermion present in the $\mathbf{2 7}$ representation of $E_{6}$. The $W_{R}$ and $h_{R}$ can then participate in the box diagram for $D^{0}-\bar{D}^{0}$ mixing[17], and can lead to large enhancements as shown in Fig. 12f.

\subsubsection{CP Violation}

CP violation in the $Q=2 / 3$ quark sector is complimentary to that of the $K$ and $B$ systems, but has yet to be explored. In the SM, the CKM phase is responsible for generating $\mathrm{CP}$ violation, and in the charm system the resulting rates are small. However, new sources of CP violating phases could greatly enhance the rates thus rendering $\mathrm{CP}$ violation in the charm system a sensitive probe for physics beyond the SM. CP violation requires the interference of at least two amplitudes with non-vanishing phases. This can occur indirectly via $D^{0}-\bar{D}^{0}$ mixing, or directly via asymmetries induced in the decay amplitude, or kinematically in final state distributions.

\section{- Indirect CP Violation}

Indirect $\mathrm{CP}$ violation corresponds to the interference of a $D^{0}$ decaying to a final state $f$ at time $t$, with a $D^{0}$ which mixes into a $\bar{D}^{0}$ and then decays to $f$ at time $t$. This process is theoretically clean as the hadronic uncertainties cancel in the asymmetry. However, since $\Delta m_{D}$ is extremely small in the SM the induced CP violation is negligible. If new physics were to enhance $D^{0}-\bar{D}^{0}$ mixing, as seen to occur in the previous section for some models, then this mechanism could yield sizeable $\mathrm{CP}$ violating effects. This interaction between mixing and $\mathrm{CP}$ violation in the $D$ meson system has recently received much attention in the literature 121, 123.

\section{- Direct CP Violation}

In order for direct $\mathrm{CP}$ violation to occur, the decay amplitudes must have two separate weak phases and two different strong phases. This can be easily seen as follows. Let us assume that the decay amplitude to final state $f$ has the form

$$
A_{f}=A_{1} e^{i \delta_{1}}+A_{2} e^{i \delta_{2}}
$$


with $A_{1,2}$ being the two amplitudes after the strong phases $\delta_{1,2}$ have been factored out. For the CP conjugate amplitude, the weak phases are conjugated, but the strong phases are not. The $\mathrm{CP}$ asymmetry is then given by

$$
\frac{\left|A_{f}\right|^{2}-\left|\bar{A}_{\bar{f}}\right|^{2}}{\left|A_{f}\right|^{2}+\left|\bar{A}_{\bar{f}}\right|^{2}}=\frac{2 \mathcal{I} m\left(A_{1}^{*} A_{2}\right) \sin \left(\delta_{1}-\delta_{2}\right)}{\left|A_{1}\right|^{2}+\left|A_{2}\right|^{2}+2 \mathcal{R e}\left(A_{1}^{*} A_{2}\right) \cos \left(\delta_{1}-\delta_{2}\right)},
$$

which clearly vanishes if $A_{1,2}$ contain the same weak phase and if $\delta_{1}=\delta_{2}$. Before estimating the typical size of this asymmetry in the SM, we first note that in contrast to $B$ decays, the branching fractions for the relevant modes, i.e., $\pi^{+} \pi^{-}, K^{+} K^{-}$, etc., are rather sizeable in the charm system, and for once, the large effects of final state interactions are welcomed! The size of the CP asymmetry in the SM is estimated [128 to be at most a few $\times 10^{-3}$. The present experimental sensitivity for various modes is in the vicinity of $10 \%$ 129.

An interesting example of the potential size of $\mathrm{CP}$ violating effects from new physics is that of left-right symmetric models [130]. In this case reasonably large values for CP asymmetries can be obtained for the Cabbibo allowed decay modes. This occurs due to the existence of an additional amplitude from the $W_{R}$ exchange, which carries a different weak phase from that of the $W_{L}$ mediated decay. The estimated values of the CP asymmetries in these models is of order a few $\times 10^{-2}$. CP asymmetries at the percent level are expected[131] in some non-minimal SUSY models for the decays $D^{0} \rightarrow K_{S}^{0} \pi^{0}, K_{S}^{0} \phi$.

\subsection{Bottom-Quark Sector}

A large amount of data on the $B$-meson system has been and will continue to be acquired during the next decade at LEP, CESR, the Tevatron, HERA, the SLAC and KEK B-factories, as well as the LHC [132, 133], and promises to yield exciting new tests of the SM. FCNC processes in the $B$-sector are not as suppressed as in the other meson systems and can occur at reasonable rates in the SM. This is due to a sizable loop-level contribution from the top-quark, which results from the combination of the large top mass (giving a big GIM splitting) and the diagonal nature of the CKM matrix. Long distance effects are expected to play less of role due to the heavy $B$ mass, and hence rare processes are essentially short distance dominated. Many classes of new models can also give significant and testable contributions to rare $B$ transitions. The benchmark process for this type of new physics search is the inclusive decay $B \rightarrow X_{s} \gamma$ (and the related exclusive process $B \rightarrow K^{*} \gamma$ ) which has been recently observed by CLEO 126]. It has since provided strong restrictions on the parameters of several theories beyond the SM [135] (which will be reviewed below). This constitutes the first direct observation of a penguin mediated process (!) and demonstrates the fertile ground ahead for the detailed exploration of the SM in rare $B$ transitions. 


\begin{tabular}{|c|c|c|}
\hline \hline Mode & SM Prediction & Experimental Bound \\
\hline$e \nu_{e}$ & $6.9 \times 10^{-12}$ & $<1.5 \times 10^{-5}(\mathrm{CLEO})$ \\
$\mu^{+} \nu_{\mu}$ & $2.9 \times 10^{-7}$ & $<2.1 \times 10^{-5}(\mathrm{CLEO})$ \\
$\tau^{+} \nu_{\tau}$ & $6.6 \times 10^{-5}$ & $<2.2 \times 10^{-3}(\mathrm{CLEO})$ \\
& & $<1.8 \times 10^{-3}(\mathrm{ALEPH})$ \\
\hline \hline
\end{tabular}

Table 13: SM branching fractions for the $B_{d}$ leptonic decay modes, assuming $f_{B}=180$ $\mathrm{MeV}$ and taking the central values of the relevant CKM matrix elements[5]. The results of experimental searches[135] are also shown.

\subsubsection{Leptonic Decays}

The SM transition rate for the purely leptonic decays $B \rightarrow \ell \nu_{\ell}$ is the same as that given for the charm system in Eq. 55, with appropriate substitutions. It is helicity suppressed and yields tiny branching fractions in the SM as shown in Table 13 . These SM predictions are somewhat imprecise due to the uncertainty in $f_{B}$ and $V_{u b}$, and hence can vary over the range (where $B_{S M}$ is the result listed in the Table)

$$
B_{S M}\left(\frac{f_{B}}{180 \mathrm{MeV}}\right)^{2}\left(\frac{V_{u b}}{0.0035}\right)^{2} .
$$

We see from the Table that the $90 \%$ C.L. experimental bounds are roughly two orders of magnitude above the SM predictions for the cases of $B \rightarrow \mu \nu_{\mu}, \tau \nu_{\tau}[134$. The B-Factories presently under construction at SLAC and KEK should be able to observe $B \rightarrow \tau \nu_{\tau}$ (and eventually the $\mu \nu_{\mu}$ mode as huge amounts of luminosity are accumulated over several years). This would provide a classic measurement of the decay constant $f_{B}$ (assuming $V_{u b}$ is known from other sources), but only if no new physics contributes to the decay. For example, in models with an enlarged Higgs sector, tree-level charged Higgs exchange can also mediate this transition. In the 2HDM of Type II the branching fraction is modified by

$$
B\left(B \rightarrow \ell \nu_{\ell}\right)=B_{S M}\left(1-\tan ^{2} \beta \frac{m_{B}^{2}}{m_{H^{ \pm}}^{2}}\right)^{2} .
$$

Taking the SM and ALEPH bound on $B \rightarrow \tau \nu_{\tau}$ given in Table 13 then implies $\tan \beta / m_{H^{ \pm}}<0.47 \mathrm{GeV}^{-1}$. This constraint varies in the range $\tan \beta / m_{H^{ \pm}}<$ $(0.38-0.68) \mathrm{GeV}^{-1}$ as one takes $f_{B}=180 \pm 40 \mathrm{MeV}$ and $\left|V_{u b}\right|=0.002-0.005$. Once this decay is detected, tests for this type of scalar exchange can be performed by measuring the helicity of the final state $\tau$. The measured branching fraction from LEP for the decay $B \rightarrow X \tau \nu$ yields 57, 134 a similar constraint of $\tan \beta / m_{H^{ \pm}}<$ $0.52 \mathrm{GeV}^{-1}$, which is independent of the uncertainties discussed above. 


\subsubsection{Radiative Decays}

As discussed above, radiative $B$ decays have become one of the best testing grounds of the SM. The CLEO Collaboration has reported [126] the observation of the inclusive decay $B \rightarrow X_{s} \gamma$ with a branching fraction of $(2.32 \pm 0.57 \pm 0.35) \times 10^{-4}$, as well as an updated measurement for the related exclusive process $B\left(B \rightarrow K^{*} \gamma\right)=$ $\left(4.3_{-1.0}^{+1.1} \pm 0.6\right) \times 10^{-5}$. This yields a value of $0.19 \pm 0.07 \pm 0.04$ for the ratio of exclusive to inclusive rates. On the theoretical side, the reliability of the calculation of the quark-level process $b \rightarrow s \gamma$ is improving [136] as agreement on the leadinglogarithmic QCD corrections has been reached and calculations at the next-toleading logarithmic order are underway. These new results have inspired a large number of investigations of this decay in various classes of models 135 .

In the SM, the quark-level transition $b \rightarrow s \gamma$ is mediated by $W$-boson and tquark exchange in an electromagnetic penguin diagram. To obtain the branching fraction, the inclusive rate is scaled to that of the semi-leptonic decay $b \rightarrow X \ell \nu$. This procedure removes uncertainties from the overall factor of $m_{b}^{5}$, and reduces the ambiguities involved with the imprecisely determined CKM factors. The result is then rescaled by the experimental value for the semi-leptonic branching fraction. The calculation of $\Gamma(b \rightarrow s \gamma)$ employs the renormalization group evolution [136] for the coefficients of the $b \rightarrow s$ transition operators in the effective Hamiltonian at the leading logarithmic level. The participating operators consist of the current-current operators $O_{1,2}$, the QCD penguin operators $O_{3-6}$, and the electro- and chromomagnetic operators $O_{7,8}$. The Wilson coefficients are evaluated perturbatively at the $W$ scale, where the matching conditions are imposed, and evolved down to the renormalization scale $\mu$, usually taken to be $\sim m_{b}$. This procedure yields

$$
B(b \rightarrow s \gamma)=\frac{6 \alpha}{\pi g(z)}\left|\frac{V_{t b} V_{t s}^{*}}{V_{c b}}\right|^{2}\left|c_{7}^{e f f}(\mu)\right|^{2} B(B \rightarrow X \ell \nu)=2.97_{-0.59}^{+0.77} \times 10^{-4}
$$

for a top-quark mass of $180 \mathrm{GeV}$, with $g(z)$ being the phase space corrections for the semi-leptonic decay. The central value corresponds to $\mu=m_{b}$, while the upper and lower errors represent the deviation due to assuming $\mu=m_{b} / 2$ and $\mu=2 m_{b}$, respectively. We see that (i) this result compares favorably to the recent CLEO measurement and (ii) the freedom of choice in the value of the renormalization scale introduces an uncertainty of order $25 \%$. Clearly, this uncertainty must be taken into account when determining constraints on new physics. Comparison with the experimental result gives the bound $\left|V_{t s} / V_{c b}\right|=0.91 \pm 0.12(\exp ) \pm 0.13(t h)$ in the SM 137.

We note here that it has been pointed out by numerous authors [138] that long distance contributions to $B \rightarrow X_{d} \gamma$ may be significant and hence these decays may not yield a good determination of the CKM element $\mid V_{t d}$. However, separate measurements of charged and neutral $B$ decays into $\rho \gamma$ and $\omega \gamma$ may be useful in sorting out the magnitude of the long distance contributions. 
Before discussing explicit models of new physics, we first investigate the constraints placed directly on the Wilson coefficients of the magnetic moment operators from the CLEO measurement of $b \rightarrow s \gamma$. Writing the coefficients at the matching scale in the form $c_{i}\left(M_{W}\right)=c_{i}\left(M_{W}\right)_{S M}+c_{i}\left(M_{W}\right)_{n e w}$, where $c_{i}\left(M_{W}\right)_{n e w}$ represents the contributions from new interactions, we see that the CLEO measurement limits the possible values of $c_{i}\left(M_{W}\right)_{\text {new }}$ for $i=7,8$. These bounds are depicted in Fig. 13(a) for $m_{t}=175 \mathrm{GeV}$, where the allowed regions lie inside the diagonal bands. We note that the two bands occur due to the overall sign ambiguity in the determination of the coefficients. The horizontal lines correspond to potential limits on $B(b \rightarrow s g)<(3-30) \times B(b \rightarrow s g)_{S M}$. We see that such a constraint on $b \rightarrow s g$ is needed to further restrict the values of the Wilson coefficients at the matching scale.

\section{- Fourth Generation}

In the case of four families there is an additional contribution to $b \rightarrow s \gamma$ from the virtual exchange of the fourth generation up-quark $t^{\prime}$ [139. The Wilson coefficients of the dipole operators are then modified by

$$
c_{7,8}\left(M_{W}\right)=c_{7,8}^{S M}\left(m_{t}^{2} / M_{W}^{2}\right)+\frac{V_{t^{\prime} b} V_{t^{\prime} s}^{*}}{V_{t b} V_{t s}^{*}} c_{7,8}^{S M}\left(m_{t}^{2} / M_{W}^{2}\right) .
$$

$V_{i j}$ represents the $4 \mathrm{x} 4 \mathrm{CKM}$ matrix which now contains nine parameters; six angles and three phases. The values of the elements of the $4 \times 4$ CKM matrix are much less restricted than their 3 generation counterparts, as one can no longer apply the 3-generation unitarity constraints [5]. Hence, even the overall CKM factor in the $b \rightarrow s \gamma$ branching ratio, $\left|V_{t b} V_{t s}^{*} / V_{c b}\right|$, can take on different values. Fig. 13(b) displays the resulting branching fraction as a function of $m_{t^{\prime}}$ for $m_{t}=180 \mathrm{GeV}$; here the vertical lines represent the range of possible values as the CKM elements are varied. These ranges were determined by generating $10^{8}$ sets of the nine parameters in the $4 \mathrm{x} 4$ CKM matrix and demanding consistency with (i) 4 generation unitarity and the extraction of the CKM elements from charged current measurements, (ii) the value of the ratio $\left|V_{u b} / V_{c b}\right|$, (iii) $\epsilon$, and (iv) $B^{0}-\bar{B}^{0}$ mixing. We see that there is little or no sensitivity to the $t^{\prime}$-quark mass, and that the CLEO measurement places additional constraints on the $4 \times 4$ CKM matrix. In fact, we find that consistency with CLEO demands $0.20 \leq\left|V_{t b} V_{t s}\right| \leq 1.5 \times 10^{-2}$ and $0.23 \leq\left|V_{t^{\prime} b} V_{t^{\prime} s}\right| \leq 1.1 \times 10^{-3}$.

\section{- Two-Higgs-Doublet Models}

In $2 \mathrm{HDM}$ the $H^{ \pm}$contributes to $b \rightarrow s \gamma$ via virtual exchange together with the top-quark. At the $W$ scale the coefficients of the dipole operators take the form (in Model II described above)

$$
c_{i}\left(M_{W}\right)=c_{i}^{S M}\left(m_{t}^{2} / M_{W}^{2}\right)+A_{1_{i}}^{H^{ \pm}}\left(m_{t}^{2} / m_{H^{ \pm}}^{2}\right)+\frac{1}{\tan ^{2} \beta} A_{2_{i}}^{H^{ \pm}}\left(m_{t}^{2} / m_{H^{ \pm}}^{2}\right),
$$


where $i=7,8$. The analytic form of the functions $A_{1_{i}}, A_{2_{i}}$ can be found in Ref. [10, 140]. In Model II large enhancements appear for small values of $\tan \beta$, but more importantly, we see that $B(b \rightarrow s \gamma)$ is always larger than that of the SM, independent of the value of $\tan \beta$ due to the presence of the $A_{1_{i}}^{H^{ \pm}}$term. In this case, the CLEO upper bound excludes [126, 141] the region to the left and beneath the curves shown in Fig. 13(c) for $m_{t}=180 \pm 12 \mathrm{GeV}$.

\section{- Supersymmetry}

There are several new classes of contributions to $b \rightarrow s \gamma$ in Supersymmetry. The large $H^{ \pm}$contributions from Model II discussed above are present, however, the limits obtained in supersymmetric theories also depend on the size of the other super-particle contributions and are generally much more complex. In particular, it has been shown 142, 143 that large contributions can arise from stop-squark and chargino exchange (due to the possibly large stop-squark mass splitting), as well as from the gluino and down-type squark loops (due to left-right mixing in the sbottom sector). The additional neutralino-down-squark contributions are expected to be small. Some regions of the parameter space can thus cancel the $H^{ \pm}$contributions resulting in predictions for the branching fraction at (or even below) the SM value, while other regions always enhance the amplitude. In minimal supergravity models with radiative breaking, the sign of the sparticle loop contributions is found to be correlated with the sign of the higgsino mass parameter $\mu[143$, 144]. This is demonstrated in Fig. 13(d) from Goto and Okada 143, where the points in this figure represent a scan of the remaining parameter space. We see that taking $\mu<0(>0)$ enhances (suppresses) the branching fraction from the predictions in the 2HDM of Type II. We also note here that $b \rightarrow s \gamma$ has been found to constrain dark matter candidates in Supersymmetric models[145].

\section{- Anomalous Trilinear Gauge Couplings}

The trilinear gauge coupling of the photon to $W^{+} W^{-}$can also be tested in radiative $B$ decays. $b \rightarrow s \gamma$ naturally avoids the problem of introducing cutoffs to regulate the divergent loop integrals due to the cancellations provided by the GIM mechanism, and hence cutoff independent bounds on anomalous couplings can be obtained. In this decay only the coefficient of the magnetic dipole operator, $\mathrm{O}_{7}$, is modified by the presence of the additional terms in Eq. 5 and can be written as

$$
c_{7}\left(M_{W}\right)=c_{7}^{S M}\left(m_{t}^{2} / M_{W}^{2}\right)+\Delta \kappa_{\gamma} A_{1}\left(m_{t}^{2} / M_{W}^{2}\right)+\lambda_{\gamma} A_{2}\left(m_{t}^{2} / M_{W}^{2}\right) .
$$

The explicit form of the functions $A_{1,2}$ can be found in Ref. [146]. As both of these parameters are varied, either large enhancements or suppressions over the $\mathrm{SM}$ prediction for the $b \rightarrow s \gamma$ branching fraction can be obtained. When one demands consistency with both the upper and lower CLEO bounds, a large region of the $\Delta \kappa_{\gamma}-\lambda_{\gamma}$ parameter plane is excluded; this is displayed in Fig. 14(a) from Ref. [126] for $m_{t}=174 \mathrm{GeV}$. Here, the allowed region is given by the cross-hatched 
Figure 13: (a) Bounds on the contributions from new physics to $c_{7,8}$. The region allowed by CLEO corresponds to the area inside the diagonal bands. The horizontal lines represent potential measurements of $R \equiv B(b \rightarrow s g) / B(b \rightarrow s g)_{S M}<30,20,10,5,3$ corresponding to the set of solid, dotted, dash-dotted, dashed, and dotted lines, respectively. The point ' $\mathrm{S}$ ' represents the SM. (b) The range of values for $B(b \rightarrow s \gamma)$ in the 4 generation SM as a function of $m_{t^{\prime}}$. (c) Limits from $b \rightarrow s \gamma$ in the charged Higgs mass $-\tan \beta$ plane. The excluded region is that to the left and below the curves. The three curves correspond to the values $m_{t}=192,180,168 \mathrm{GeV}$ from top to bottom. (d) $B(b \rightarrow s \gamma)$ as a function of the charged Higgs mass with $m_{t}=175 \mathrm{GeV}$ and $\tan \beta=5$ from Ref. 143. The solid curve corresponds to the $2 \mathrm{HDM}$ Model II value, while the dashed-dot curve represents the SM. Each dot corresponds to a sample point of the SUSY parameter space. 
area, where the white strip down the middle is excluded by the lower bound and the outer white areas are ruled out by the upper limit on $B(b \rightarrow s \gamma)$. The ellipse represents the region allowed by D0 147]. Note that the SM point in the $\Delta \kappa_{\gamma}-\lambda_{\gamma}$ plane (labeled by the dot) lies in the center of one of the allowed regions. We see that the collider constraints are complementary to those from $b \rightarrow s \gamma$.

Figure 14: (a) Constraints on anomalous $W W \gamma$ couplings. The shaded area is that allowed by CLEO and the interior of the ellipse is the region allowed by D0. The dot represents the SM values. (b) Bounds on anomalous top-quark photon couplings from $b \rightarrow s \gamma$. The solid and dashed curves correspond to the cases described in the text. In each case, the allowed regions lie inside the semi-circles.

\section{- Anomalous Top-Quark Couplings}

If the top-quark has anomalous couplings to on-shell photons or gluons, the rate for $b \rightarrow s \gamma$ would be modified. The effect of an anomalous magnetic and/or electric dipole moment in the Lagrangian of Eq. 6 on the Wilson coefficients is

$$
c_{7,8}\left(M_{W}\right)=c_{7,8}^{S M}\left(m_{t}^{2} / M_{W}^{2}\right)+\kappa_{\gamma, g} F_{17,8}\left(m_{t}^{2} / M_{W}^{2}\right)+\tilde{\kappa}_{\gamma, g} F_{2_{7,8}}\left(m_{t}^{2} / M_{W}^{2}\right) .
$$

The functions $F_{1,2}$ can be found in Ref. [148]. The effects of anomalous chromodipole moments arise from operator mixing. When the resulting branching fraction and the CLEO data are combined, the constraints in Fig. 14(b) are obtained for $m_{t}=180 \mathrm{GeV}$. In this figure, the allowed region is given by the area inside the solid (dashed) semi-circle when $\kappa_{g}, \tilde{\kappa}_{g}=0\left(=\kappa_{\gamma}, \tilde{\kappa}_{\gamma}\right)$. These bounds are considerably weaker than those obtainable from direct top-quark production at colliders [80]. 


\subsubsection{Other Rare Decays}

Other FCNC decays of $B$ mesons include $B_{d, s}^{0} \rightarrow \ell^{+} \ell^{-}, \gamma \gamma, B \rightarrow X_{s, d}+\ell^{+} \ell^{-}, X_{s, d} \nu \bar{\nu}$, with $\ell=e \mu \tau$. In the SM they are mediated by appropriate combinations of electromagnetic and weak penguins as well as box diagrams, and generally have larger rates, as discussed above, due to the heavy top-quark and the diagonal nature of the CKM matrix. The SM predictions [149] and current experimental situation[5, 132, 150 for these decays are summarized in Table 14, taking $m_{t}=180 \mathrm{GeV}$. The purely leptonic decays, $B^{0} \rightarrow \ell^{+} \ell^{-}$can be enhanced by contributions from new physics at both the loop-level, for example in Extended Technicolor models 152 or by virtual $H^{ \pm}$exchange [153] in 2HDM, and at tree-level, e.g., with leptoquark exchange 18]. However, as can be seen from the Table, the experimental probes of these purely leptonic decays are orders of magnitude above the expected rates, and hence only potentially large tree-level contributions can currently be tested. Indeed, the most stringent constraints on tree-level leptoquark contributions in $B$ decays are obtained from the exclusive reaction $B \rightarrow K e \mu[18$. However, in this case there exist large uncertainties associated with the hadronic matrix elements, yielding some sloppiness in the resulting bounds.

The transition $b \rightarrow s \ell^{+} \ell^{-}$merits further attention as it offers an excellent opportunity to search for new physics. For example, it has been found 154 that Extended Technicolor models with a GIM mechanism already violates (!) the experimental upper bound on $B \rightarrow X_{s} \mu \mu$, but more traditional ETC models yield a rate which is close to the SM prediction. The decay proceeds via electromagnetic and $Z$ penguin as well as by $W$ box diagrams and hence can probe different coupling structures than the pure electromagnetic process $b \rightarrow s \gamma$. The matrix element can be written as

$\mathcal{M}=\frac{\sqrt{2} G_{F} \alpha}{\pi} V_{t b} V_{t s}^{*}\left[c_{9}^{e f f} \bar{s}_{L} \gamma_{\mu} b_{L} \bar{\ell} \gamma^{\mu} \ell+c_{10} \bar{s}_{L} \gamma_{\mu} b_{L} \bar{\ell} \gamma^{\mu} \gamma_{5} \ell-2 c_{7} m_{b} \bar{s}_{L} i \sigma_{\mu \nu} \frac{q^{\nu}}{q^{2}} b_{R} \bar{\ell} \gamma^{\mu} \ell\right]$

where $q^{2}$ is the momentum transferred to the lepton pair. Here we take the sign convention of Ali et al. 155 for the Wilson coefficients. These short distance contributions are theoretically well-known as the NLO QCD corrections have recently been computed [156] for the coefficient $c_{9}$, and $c_{10}$ does not receive any large contributions from the renormalization evolution. This reaction also receives long distance contributions from the processes $B \rightarrow K^{(*)} \psi^{\left({ }^{\prime}\right)}$ followed by $\psi^{\left({ }^{\prime}\right)} \rightarrow \ell^{+} \ell^{-}$ and from $c \bar{c}$ continuum intermediate states. The short distance contributions lead to the inclusive branching fractions given in the Table; we see that these modes will likely be observed during the next few years! The best technique of separating the long and short distance contributions, as well as observing any deviations from the SM predictions, is to measure the various kinematic distributions associated with the final state lepton pair, such as the lepton pair invariant mass distribution [157], the lepton pair forward-backward asymmetry [155], and the tau 


\begin{tabular}{|c|c|c|}
\hline Decay Mode & Experimental Limit & 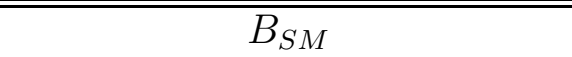 \\
\hline$B_{d}^{0} \rightarrow e^{+} e^{-}$ & $<5.9 \times 10^{-6}(\mathrm{CLEO})$ & $2.6 \times 10^{-15}$ \\
\hline$B_{d}^{0} \rightarrow \mu^{+} \mu^{-}$ & $<1.6 \times 10^{-6}(\mathrm{CDF})$ & $1.1 \times 10^{-10}$ \\
\hline$B_{d}^{0} \rightarrow \tau^{+} \tau^{-}$ & - & $2.1 \times 10^{-8}$ \\
\hline$B_{s}^{0} \rightarrow e^{+} e^{-}$ & - & $5.3 \times 10^{-14}$ \\
\hline$B_{s}^{0} \rightarrow \mu^{+} \mu^{-}$ & $<8.4 \times 10^{-6}(\mathrm{CDF})$ & $2.4 \times 10^{-9}$ \\
\hline$B_{s}^{0} \rightarrow \tau^{+} \tau^{-}$ & - & $5.1 \times 10^{-7}$ \\
\hline$B^{0} \rightarrow e^{ \pm} \mu^{\mp}$ & $<5.9 \times 10^{-6}(\mathrm{CLEO})$ & 0 \\
\hline$B^{0} \rightarrow e^{ \pm} \tau^{\mp}$ & $<5.3 \times 10^{-4}(\mathrm{CLEO})$ & 0 \\
\hline$B^{0} \rightarrow \mu^{ \pm} \tau^{\mp}$ & $<8.3 \times 10^{-4}(\mathrm{CLEO})$ & 0 \\
\hline$B_{d}^{0} \rightarrow \gamma \gamma$ & $<3.8 \times 10-5($ L3 $)$ & $1.0 \times 10^{-8}$ \\
\hline$B_{s}^{0} \rightarrow \gamma \gamma$ & $<1.1 \times 10^{-4}(\mathrm{~L} 3)$ & $3 \times 10^{-7}$ \\
\hline$B \rightarrow X_{s}+\gamma$ & $(2.32 \pm 0.57 \pm 0.35) \times 10^{-4}(\mathrm{CLEO})$ & $\left(2.97_{-0.59}^{+0.77}\right) \times 10^{-4}$ \\
\hline$B \rightarrow K^{*} \gamma$ & $\left(4.3_{-1.0}^{+1.1} \pm 0.6\right) \times 10^{-5}(\mathrm{CLEO})$ & $(4.0 \pm 2.0) \times 10^{-5}$ \\
\hline$B^{+} \rightarrow \rho^{+} \gamma$ & $<0.34 \times B\left(B \rightarrow K^{*} \gamma\right)(\mathrm{CLEO})$ & $(1.9 \pm 1.6) \times 10^{-6}$ \\
\hline$B^{0} \rightarrow \rho^{0}(\omega) \gamma$ & $<0.34 \times B\left(B \rightarrow K^{*} \gamma\right)(\mathrm{CLEO})$ & $(0.85 \pm 0.65) \times 10^{-6}$ \\
\hline$B \rightarrow X_{s}+e^{+} e^{-}$ & - & $7.0 \times 10^{-6}$ \\
\hline$B \rightarrow X_{s}+\mu^{+} \mu^{-}$ & $<5.0 \times 10^{-5}(\mathrm{UA} 1)$ & $6.2 \times 10^{-6}$ \\
\hline$B \rightarrow X_{s}+\tau^{+} \tau^{-}$ & - & $3.2 \times 10^{-7}$ \\
\hline$B^{0} \rightarrow K^{0} e e / \mu \mu$ & $<1.5 / 2.6 \times 10^{-4}(\mathrm{CLEO})$ & $(5.0 \pm 3.0) /(3.0 \pm 1.8) \times 10^{-7}$ \\
\hline$B^{-} \rightarrow K^{-} e e / \mu \mu$ & $<1.2 / 0.9 \times 10^{-5}(\mathrm{CLEO})$ & $(5.0 \pm 3.0) /(3.0 \pm 1.8) \times 10^{-7}$ \\
\hline $\bar{B}^{0} \rightarrow \bar{K}^{* 0} e e / \mu \mu$ & $<1.6 / 2.5 \times 10^{-5}(\mathrm{CLEO} / \mathrm{CDF})$ & $(2.0 \pm 1.0) /(1.25 \pm 0.62) \times 10^{-6}$ \\
\hline $\bar{B}^{-} \rightarrow \bar{K}^{*-} e e / \mu \mu$ & $<6.3 / 11 \times 10^{-4}(\mathrm{CLEO})$ & $(2.0 \pm 1.0) /(1.25 \pm 0.62) \times 10^{-6}$ \\
\hline$B^{+} \rightarrow K^{+} e^{ \pm} \mu^{\mp}$ & $<1.2 \times 10^{-5}(\mathrm{CLEO})$ & 0 \\
\hline $\bar{B}^{0} \rightarrow \bar{K}^{* 0} e^{ \pm} \mu^{\mp}$ & $<2.7 \times 10^{-5}(\mathrm{CLEO})$ & 0 \\
\hline$B \rightarrow X_{s}+\nu \bar{\nu}$ & $<3.9 \times 10^{-4} \dagger$ & $5.0 \times 10^{-5}$ \\
\hline
\end{tabular}

Table 14: Standard Model predictions[149] for the branching fractions for various rare $B$ meson decays with $f_{B_{d}}=180 \mathrm{MeV}$. Also shown are the current experimental limits[5,150]. $\dagger$ This is an inferred bound[151] from limits on $B \rightarrow \tau \nu_{\tau}$. 
polarization asymmetry 158 in the case $\ell=\tau$. These distributions are presented in Fig. 15, with and without the resonance contributions. Note that both asymmetries are large for this value of the top-quark mass. As an example of how new physics can affect this process, we display in Fig. 15(d) the tau polarization asymmetry for various changes of sign of the contributing Wilson coefficients. Measurement of all three kinematic distributions would allow for the determination of the sign and magnitude of the Wilson coefficients for the contributing electroweak loop operators and thus provide a completely model independent analysis. We present a 95\% C.L. Monte Carlo fit [158] to these coefficients in Fig. 16, assuming the $\mathrm{SM}$ is realized in nature, and taking an integrated luminosity of $5 \times 10^{8} \mathrm{~B} \bar{B}$ pairs. (This clearly requires the high statistics samples which will be available at future B-factories.) This procedure demonstrates that the coefficients $c_{7,9,10}(\mu)$ can be measured to an accuracy of roughly $7.5 \%, 15 \%$, and $5 \%$, respectively, which would yield a very stringent test of the SM.

Presently, there have been no direct searches for the 'invisible' decay, $B \rightarrow$ $X_{s} \nu \bar{\nu}$, however, bounds on this process may be inferred from searching for events with large missing energy in $B$ decays, such as $B \rightarrow \tau \nu_{\tau}$. The limit obtained in this manner 151] is quoted in Table 14. This transition proceeds via $Z$ penguin and $W$ box diagrams in the SM, with the rate being roughly one order of magnitude lower than the inferred bound. Various classes of new interactions can contribute substantially to this decay and they have been categorized in Ref. [151]; these include models with leptoquarks, Supersymmetry with R-parity violating couplings, Topcolor models, and horizontal gauge symmetries. Defining a most general form of a four-fermion interaction responsible for this decay as $\mathcal{L}=C_{L} \bar{s}_{L} \gamma_{\mu} b_{L} \bar{\nu}_{L} \gamma^{\mu} \nu_{L}+C_{R} \bar{s}_{R} \gamma_{\mu} b_{R} \bar{\nu}_{L} \gamma^{\mu} \nu_{L}$, gives

$$
B\left(B \rightarrow X_{s} \nu \bar{\nu}\right)=\frac{C_{L}^{2}+C_{R}^{2}}{\left|V_{c b}\right|^{2} g(z)} B(B \rightarrow X \ell \nu),
$$

from which these authors have found the model independent bound

$$
C_{L}^{2}+C_{R}^{2}<3.0 \times 10^{-6}\left[\frac{B\left(B \rightarrow X_{S} \nu \bar{\nu}\right)}{3.9 \times 10^{-4}}\right] .
$$

In some models, the restrictions obtained from this process either surpass or are competitive with those from $B \rightarrow X_{s} \ell^{+} \ell^{-}$.

\subsection{4 $B^{0}-\bar{B}^{0}$ Mixing}

The quark level process which is dominantly responsible for $B^{0}-\bar{B}^{0}$ mixing in the $\mathrm{SM}$ is that of top-quark exchange in a $W$ box diagram. The mass difference for $B_{d}$ meson mixing is then given by

$$
\Delta M_{d}=\frac{G_{F}^{2} M_{W}^{2} m_{B}}{6 \pi^{2}} f_{B_{d}}^{2} B_{B_{d}} \eta_{B_{d}}\left|V_{t b} V_{t d}^{*}\right|^{2} F\left(m_{t}^{2} / M_{W}^{2}\right)
$$


Figure 15: (a) Differential branching fraction, (b) lepton pair forward backward asymmetry, and (c) tau polarization asymmetry as a function of $\hat{s}$ for $\ell=\tau$ (solid and dashed curves) and $\ell=e$ (dotted and dash-dotted curves), with and without the long distance contributions. (d) Tau polarization asymmetry with changes in sign of the Wilson coefficients at the electroweak scale, corresponding to $c_{10}, c_{9}, c_{9,10}, S M$, and $c_{7,8}$ from bottom to top. 
Figure 16: $95 \%$ C.L. projected contour in the (a) $c_{9}-c_{10}$ and (b) $c_{7}-c_{10}$ plane. 'S' labels the SM prediction and the diamond represents the best fit values. Here we use the sign convention of $\operatorname{Ali}[155]$ for the coefficients.

with $\eta_{B_{d}}$ being the QCD correction factor which is calculated to NLO [159], and F(x) being the usual Inami-Lim function [118]. An equivalent expression for $B_{s}$ mixing is obtained with $d \rightarrow s$. This yields the SM values of $\Delta M_{d}=\left(3.0_{-2.7}^{+9.0}\right) \times 10^{-13}$ $\mathrm{GeV}$ and $\Delta M_{s}=\left(7.4_{-4.3}^{+8.6}\right) \times 10^{-12} \mathrm{GeV}$, where the ranges correspond to taking $m_{t}^{\text {phys }}=180 \pm 12 \mathrm{GeV},\left|V_{t d}\right|=0.009 \pm 0.005$ and $\left|V_{t s}\right|=0.040 \pm 0.006$ as given in Ref. [5], and $f_{B_{d}} \sqrt{B_{B_{d}}}=180 \pm 40 \mathrm{MeV}, f_{B_{s}} \sqrt{B_{B_{s}}}=200 \pm 40 \mathrm{MeV}$ as suggested by lattice gauge theory 160. This agrees well with the experimental bounds 161 of $\Delta M_{d}=(3.01 \pm 0.13) \times 10^{-13} \mathrm{GeV}$ and $\Delta M_{s}>4.0 \times 10^{-12} \mathrm{GeV}$. This situation is summarized in Fig. 17.

The ratio of hadronic matrix elements, $f_{B_{d}} \sqrt{B_{B_{d}}} / f_{B_{s}} \sqrt{B_{B_{s}}}$, is more accurately calculable in lattice gauge theory 160 , hence a measurement of $\Delta M_{d} / \Delta M_{s}$ would be an important determination of the value of the CKM ratio $\left|V_{t d} / V_{t s}\right|$ in the SM. Remarkably, this remains true in many scenarios beyond the SM. In this class of models, the virtual exchange of new particles alters the Inami-Lim function in Eq. 77 above, but not the factors in front of the function. The effects of the new physics then cancels in the ratio. Models of this type include, 2HDM and Supersymmetry in the super-CKM basis. Notable exceptions to this feature can be found in models which (i) change the structure of the CKM matrix, such as the addition of a fourth generation, or extra singlet quarks, and in Left-Right Symmetric models, (ii) have couplings proportional to fermion masses, such as flavor changing Higgs models, or (iii) have generational dependent couplings, e.g., leptoquarks or SUSY with 
R-parity violation.

Figure 17: The SM expectation for the $\Delta M_{d}-\Delta M_{s}$ plane, where the predicted region lies inside the solid curves. The experimental bounds lie in between the solid horizontal lines and to the right of the solid vertical line.

It is difficult to use $\Delta M_{d}$ alone to restrict new physics due to the enormous errors on the theoretical predictions for this quantity from the imprecisely determined CKM factors and unmeasured $B$ hadronic matrix elements. (This is unfortunate as $\Delta M_{d}$ is so precisely measured!) In most cases, the restrictions obtained from $b \rightarrow s \gamma$ surpass those from $B^{0}-\bar{B}^{0}$ mixing. As a demonstration of this point, we note the results in Ref. [162] where $\Delta M_{d}$ is calculated in 2HDM of Type II and in minimal Supergravity models. These models contribute to $B^{0}-\bar{B}^{0}$ mixing via $H^{ \pm}$ top-quark, chargino-stop-squark, and gluino-down-squark virtual exchange in box diagrams (the neutralino contributions are found to be small). These authors find that although substantial enhancements are possible (up to a factor of $50 \%$ over the SM), $\Delta M_{d}$ remains well within the overall theoretical errors. Another example is given in Fig. 13(b), where $b \rightarrow s \gamma$ is shown to greatly restrict the parameter space of the 4 generation $\mathrm{SM}$, even after constraints from $B^{0}-\bar{B}^{0}$ mixing were applied.

\subsubsection{CP Violation in $B$ Decays}

$\mathrm{CP}$ violation in the $B$ system will be examined [133] during the next decade at dedicated B-Factories. CP violation arises in the SM from the existence of the phase in the 3 generation CKM matrix as first postulated by Kobayashi and Maskawa [163]. The relation $V_{t b} V_{t d}^{*}+V_{c b} V_{c d}^{*}+V_{u b} V_{u d}^{*}=0$, which is required by unitarity, can be 
depicted as a triangle in the complex plane as shown in Fig. 18, where the area of the triangle represents the amount of $\mathrm{CP}$ violation. It can be shown that the apex of the triangle is located at the point $(\rho, \eta)$ in the complex plane, where $\rho$ and $\eta$ are parameters describing the CKM matrix in the Wolfenstein notation[164]. The present status of these parameters is summarized in Fig. 19(a), where the shaded area is that allowed in the SM. This region is determined by measurements of the quantities (i) $\left|V_{u b}\right|$ and $\left|V_{c b}\right|$, (ii) $\epsilon_{K}$, and (iii) the rate for $B_{d}^{0}-\bar{B}_{d}^{0}$ mixing, together with theoretical estimates for the parameters which relate these measurements to the underlying theory, such as $B_{K}, f_{B}$, and $B_{B}$. The value of $\overline{m_{t}}\left(m_{t}\right)$ is taken to be consistent with the physical range $180 \pm 12 \mathrm{GeV}$. This yields the allowed ranges for the angles of the triangle: $-0.89 \leq \sin 2 \alpha \leq 1.00,0.18 \leq \sin 2 \beta \leq 0.81$, and $-1.00 \leq \sin 2 \gamma \leq 1.00$.

Figure 18: The rescaled Unitarity triangle.

It is important to remember that this picture can be dramatically altered if new physics is present, even if there are no new sources of CP violation. Figure 19(b) displays the constraints in the $\rho-\eta$ plane in the two-Higgs-doublet Model II. In this case the presence of the extra Higgs doublet is felt by the virtual exchange of the $H^{ \pm}$ boson in the box diagram which mediates $B_{d}^{0}-\bar{B}_{d}^{0}$ mixing and governs the value of $\epsilon_{K}$. For this $\rho-\eta$ region, the allowed ranges of the angles of the unitarity triangle become $-1.00 \leq \sin 2 \alpha \leq 1.00,0.12 \leq \sin 2 \beta \leq 0.81$, and $-1.00 \leq \sin 2 \gamma \leq$ 1.00. In fact, this opens up a new allowed region in the $\sin 2 \alpha-\sin 2 \beta$ plane, as shown in Fig. 20 from Ref. [165. Similar effects have also been pointed out in Supersymmetric models 166. We see that the SM predictions for CP violation are thus modified. Clearly, caution must be exercised when relating the results of future $\mathrm{CP}$ violation experiments to the $\rho-\eta$ plane.

The B-Factories presently under construction should be able to discern whether new physics contributes to CP violation. Signals for new sources of CP violation include, (i) non-closure of the 3 generation unitarity triangle, (ii) new contributions to $B^{0}-\bar{B}^{0}$ mixing which yield a non-vanishing phase for this process, (iii) non- 
Figure 19: Constraints in the (a) SM and (b) two-Higgs-doublet Model II in the $\rho-\eta$ plane from $\left|V_{u b}\right| /\left|V_{c b}\right|$ (dotted circles), $B_{d}^{0}-\bar{B}_{d}^{0}$ mixing (dashed circles) and $\epsilon$ (solid hyperbolas). The shaded area corresponds to that allowed for the apex of the Unitarity triangle.

vanishing $\mathrm{CP}$ asymmetries for the channels $B_{d}^{0} \rightarrow \phi \pi^{0}, K_{S}^{0} K_{S}^{0}$, (iv) inconsistency of separate measurements of the angles of the unitarity triangle, and (v) a deviation of CP rates from SM predictions. Models which contain additional CP phases include, non-minimal Supersymmetry, Multi-Higgs Doublets, Left-Right Symmetric Models, and the Superweak Model. A concise review of the effects of these models on $\mathrm{CP}$ violating observables is given by Nir 167. We present here, as an example, the case of Multi-Higgs models with three or more Higgs doublets. In this scenario $B^{0}-\bar{B}^{0}$ mixing receives additional contributions from the $H_{1,2}^{ \pm}$exchange which depend on the phase in the charged scalar mixing matrix (this phase is discussed in Eq. 2). Interference between these contributions and the SM yield an overall non-zero phase in $\Delta M_{d}$. Denoting this phase as $\theta_{H}$ the unitarity angles measured by $\mathrm{CP}$ asymmetries in $B$ decays are thus shifted by

$$
a_{C P}\left(B \rightarrow \psi K_{S}\right)=-\sin \left(2 \beta+\theta_{H}\right), a_{C P}(B \rightarrow \pi \pi)=\sin \left(2 \alpha+\theta_{H}\right) .
$$

The magnitude of this effect depends on the size of $\theta_{H}$, which has recently 165 been constrained by $b \rightarrow s \gamma$. Another interesting example is provided in models with an extra iso-singlet down quark; in this scenario, it has been found 168] that measurements of the unitarity angles $\alpha$ and $\beta$ alone are not enough to distinguish and bound the new contributions, and that observation of both the third angle $\gamma$ and $B_{s}$ mixing are also needed. In summary, the large data sample which will 
become available will provide a series of unique consistency tests of the quark sector and will challenge the SM in a new and quantitatively precise manner.

Figure 20: The allowed region in the $\sin 2 \alpha-\sin 2 \beta$ plane in the SM (solid) and in 2HMD (dot-dashed). From [165].

\subsection{Top-Quark}

Loop induced flavor changing top-quark decays are small in the SM, as in the charm-quark system, due to the effectiveness of the GIM mechanism and the small masses of the $Q=-1 / 3$ quarks. However, these transitions are anticipated to be theoretically clean as long distance effects are expected to be negligible. The SM rates for $t \rightarrow c \gamma, c Z, c g$ are given by $4.9 \times 10^{-13}, 1.4 \times 10^{-13}, 4.4 \times 10^{-11}$, respectively, for $m_{t}=180 \mathrm{GeV} 169$. The branching fraction for $t \rightarrow c h$ as a function of the Higgs mass is represented by the solid curve in Fig. 21(a-b). We see that this rate is also tiny, being in the $10^{-13}$ range over the entire kinematically allowed region for the Higgs mass. Loop contributions from new physics have been studied in 2HDM[169, [70] and in SUSY [171], and generally can enhance these transition rates by 3-4 orders of magnitude for some regions of the parameter space. The effects of virtual $H^{ \pm}$exchange in 2HDM of Type II on the reactions $t \rightarrow \mathrm{cV}$, $V=\gamma, Z, g$, are displayed in Fig. 21(c) for $m_{t}=180 \mathrm{GeV}$. We see that, indeed, enhancements are present for large values of $\tan \beta$. We also examine the decays $t \rightarrow c h, c H$ in Model II, where $h$ and $H$ respectively represent the lightest and heaviest physical neutral scalars present in 2HDM. The resulting rates are depicted in Fig. 21(a-b) for the demonstrative case of $m_{H^{ \pm}}=600 \mathrm{GeV}$ and $\tan \beta=2(30)$, corresponding to the dashed (solid) curves. Here we have made use of the SUSY Higgs mass relationships in order to reduce the number of free parameters. We note that the effects of super-partner virtual exchange should also be included (with, of course, a corresponding increase in the number of parameters!). We have also studied these modes in Model I, and found similar rate increases for regions of the parameter space. Even if new physics were to produce such enhancements, 
the resulting branching fractions would still lie below the observable level in future experiments at an upgraded Tevatron, the LHC, or the NLC.

On the other hand, if these FCNC decays were to be detected, they would provide an indisputable signal for new physics. Hence a model independent approach in probing anomalous FCNC top-quark couplings has recently been taken by a number of authors 172. By parameterizing the general $t c V$ vertex in a manner similar to that presented in Eq. 6, and performing a Monte Carlo study of the signal rate versus potential backgrounds, Han et al. 172 have found that such anomalous couplings can be probed down to the level of $\left.\kappa_{\gamma, Z} \equiv \sqrt{g_{L}^{2}+g_{R}^{2}}\right|_{\gamma, Z} \simeq 0.1(0.01)$ at the Tevatron (LHC). This corresponds to values of the branching fractions for $t \rightarrow c Z, c \gamma$ at the level of few $\times 10^{-3}$ for the Tevatron bounds and $10^{-4}$ for the LHC. CDF has, in fact, already performed a search for these FCNC decays from their present top sample, and has placed the bounds 173 $B(t \rightarrow c \gamma+u \gamma)<2.9 \%$ and $B(t \rightarrow c Z+u Z)<90 \%$ at $95 \%$ and $90 \%$ C.L., respectively.

Potential non-SM tree-level decays of the top-quark could feasibly occur at measurable rates in future colliders. Examples of these possible transitions are: (i) the decay of top into a charged Higgs, $t \rightarrow b H^{+}$in multi-Higgs models [174, (ii) the tree-level flavor-changing decay $t \rightarrow c h$, which can occur, if kinematically accessible, in multi-higgs models without natural flavor conservation 12, 175, (iii) $t \rightarrow \tilde{t} \tilde{\chi}^{0}$ which can take place in Supersymmetry if the stop-squark is sufficiently light 176 (this possibility is related to the large value of the top Yukawa coupling, and is thus special to the top system), and (iv) $t \rightarrow \tilde{\ell}^{+} d$ in SUSY models with Rparity violation 177]. For favorable values of the parameters, each of these modes could be competitive with the SM decay $t \rightarrow b W^{+}$. The observation of the topquark by CDF and D0, which relies heavily on the expected signal from SM top decay [30], can thus restrict the values of the branching fractions for these potential new modes. The possible constraints that could be obtained on the models which would allow the decays (i) $t \rightarrow b H^{+}$and (ii) $t \rightarrow c h$ to occur, if these collaborations were to make the statement that the observed $t \bar{t}$ production rate is $50-90 \%$ of that expected in the SM are given in Fig. 22. We have examined the case of the decay into a $H^{ \pm}$in Model II, taking $m_{t}=180 \mathrm{GeV}$, and find that the potentially excluded regions lie below the curves. Clearly, large regions of the parameter space have the potential to be ruled out. In the case of $t \rightarrow c h$ decay, we have parameterized the tree-level tch coupling as $\left(\sqrt{2} G_{F}\right)^{1 / 2} m_{t}\left(\alpha-\beta \gamma_{5}\right)$ and displayed the restrictions in the $k \equiv \sqrt{\alpha^{2}+\beta^{2}}-m_{h}$ plane. The region above the curves would be excluded.

$\mathrm{CP}$ violation in top-quark production and decay is expected to be very small in the SM 178, however, numerous models with new interactions, such as multiHiggs models and Supersymmetry, can give rise to $\mathrm{CP}$ violation in the top system at interesting levels. Since the top-quark decays before it has time to hadronize, it provides a particularly good laboratory for the study of such effects. Searches for $\mathrm{CP}$ violating effects can be carried out by studying CP-odd spin-momentum correlations in the top-quark decay products. $e^{+} e^{-}$colliders, with polarized beams, 
Figure 21: Branching fractions for (a) $t \rightarrow c h$ (b) $t \rightarrow c H$ as a function of the neutral Higgs mass in 2HDM of Type II. The SM rate is represented by the solid curve. (c) $B(t \rightarrow c V)$ where $V=g, \gamma, Z$ as a function of $\tan \beta$ in Model II. In all cases the topquark mass is taken to be $180 \mathrm{GeV}$. 
are especially suited to carry out such investigations. Numerous studies of CP symmetry tests can be found in Ref. [80, 179].

Figure 22: Constraints placed on the non-standard decays (a) $t \rightarrow b H^{+}$and (b) $t \rightarrow c h$ from demanding that the observed event rate for top-quark pair production is at least $50,60,70,80,90 \%$ of that expected in the SM, corresponding to the dashed-dot, solid, dotted, dashed, and solid curves. $m_{t}=180 \mathrm{GeV}$ is assumed.

\section{$7 \quad$ Electric Dipole Moments}

Experiments sensitive to the electric dipole moments (EDMs) of atoms 180, 181, 182, 183, molecules [184, and the neutron [185], provide by far the most sensitive tests of low energy flavor conserving CP violation 186, 187, 188. The current experimental bounds given in Table 15 represent an extraordinary level of precision. New techniques, such as atomic traps, may allow improvements of up to two orders of magnitude by the turn of the century. Although no EDM has yet been observed, as discussed below, the current bounds already place stringent limits on $\mathrm{CP}$ violating extensions of the SM.

The SM possesses two possible sources of $\mathrm{CP}$ violation: the phase in the CKM quark mixing matrix, and the QCD vacuum angle, $\bar{\theta}_{Q C D}$. The CKM phase contributes to EDMs only at three loops, and requires mixings through all three generations. As such, it is highly suppressed, and gives contributions well below current experimental sensitivity. In contrast, the QCD vacuum angle contribution is not suppressed, and represents a potential background to any non-standard model con- 


\begin{tabular}{|c|c|c|}
\hline \hline Particle & EDM $(e \mathrm{~cm})$ & Reference \\
\hline${ }^{133} \mathrm{Cs}$ & $<7 \times 10^{-24}$ & $\mathbb{1 8 0}$ \\
${ }^{205} \mathrm{Tl}$ & $<2 \times 10^{-24}$ & 181 \\
${ }^{129} \mathrm{Xe}$ & $<1 \times 10^{-26}$ & 182 \\
${ }^{199} \mathrm{Hg}$ & $<9 \times 10^{-28}$ & 1183 \\
${ }^{205} \mathrm{TlF}$ & $<5 \times 10^{-23}$ & 1184 \\
neutron & $<8 \times 10^{-26}$ & $\mathbb{1 8 5}$ \\
\hline \hline
\end{tabular}

Table 15: Experimental bounds on electric dipole moments.

tributions. However, as discussed below, positive measurements in the atomic or molecular systems could distinguish $\bar{\theta}_{Q C D}$ from non-standard model CP violation.

A systematic determination of the limits placed on $\mathrm{CP}$ violating extensions of the SM from experimental bounds requires evaluating the effective $\mathrm{CP}$ violating interactions at the weak, nuclear, and atomic scales. The results of such an analysis indicate that, in general, atoms with an unpaired electron $\left({ }^{133} \mathrm{Cs}\right.$ and $\left.{ }^{205} \mathrm{Tl}\right)$ are most sensitive to weak sector $\mathrm{CP}$ violation, while atoms and molecules with paired electrons $\left({ }^{129} \mathrm{Xe},{ }^{199} \mathrm{Hg}\right.$, and $\left.{ }^{205} \mathrm{TlF}\right)$ are sensitive to strong sector CP violation [187. At the atomic scale, the dominant weak sector $\mathrm{CP}$ violation is from the electron EDM. In the strong sector, for nuclear spin $j=\frac{1}{2}$, the most important interaction is the electric dipole moment of the nucleus, which gives rise to a local electromagnetic interaction between the electrons and nucleus, generally referred to as the Schiff moment. For nuclear spin $j \geq 1$ the most important strong sector interaction is the nuclear magnetic quadrapole moment. At the nuclear scale both these interactions arise predominantly from the CP-odd pion-nucleon coupling, and at the microscopic scale from the light quark chromo-electric dipole moment (CDM) [187. The disparate sensitivity of atoms and molecules with and without net electronic spin allows $\mathrm{CP}$ violation in the weak and strong sectors to be distinguished. To illustrate this, the relative EDMs which arise from an electron EDM and CP-odd pion nucleon coupling (assumed for simplicity to be isoscalar) are given in Table 16. For simplicity the strong sector contributions are normalized to that of ${ }^{199} \mathrm{Hg}$, for which the best experimental bound is available. Notice that all the atoms, independent of electronic spin, are roughly equally sensitive to $\mathrm{CP}$ violation which arises in the strong sector. The exception is ${ }^{129} \mathrm{Xe}$ for which the atomic matrix elements are suppressed because of the closed electron shell. The slightly increased sensitivity for ${ }^{133} \mathrm{Cs}$ with nuclear spin $j=\frac{7}{2}$ is due to the nuclear magnetic quadrapole moment, which does not exist in the other atoms with nuclear spins $j=\frac{1}{2}$. The highly increased sensitivity of the molecule ${ }^{205} \mathrm{TlF}$ relative to the atoms is due to the small energy splitting for rotational levels of opposite parity. This is offset, however, by increased sensitivity to experimental systematics. 


\begin{tabular}{|c|c|c|}
\hline \hline Particle & Weak & Strong \\
\hline${ }^{133} \mathrm{Cs}$ & 120 & 5 \\
${ }^{205} \mathrm{Tl}$ & -600 & 0.5 \\
${ }^{129} \mathrm{Xe}$ & -0.0008 & 0.09 \\
${ }^{199} \mathrm{Hg}$ & 0.012 & 1 \\
${ }^{205} \mathrm{TlF}$ & 80 & 2000 \\
neutron & - & 150 \\
\hline \hline
\end{tabular}

Table 16: Relative sensitivity of EDMs to CP violation arising in the weak sector (electron EDM) and strong sector (CP-odd pion-nucleon coupling).

Extensions of the SM at or just above the weak scale often contain additional CP violation. This generally gives contributions to EDMs which are not accidentally suppressed, as is the CKM contribution. As an example, supersymmetric theories generally possess a large number of phases in the most general soft supersymmetry breaking terms. The magnitude of these phases depends on the CP properties of the supersymmetry breaking sector. It has recently been emphasized that even the CKM phase can induce non-zero supersymmetric phases at the electroweak scale from renormalization group running between the Planck and GUT scales [189. The existence of $\mathrm{CP}$ violating supersymmetric phases is therefore an important test of supersymmetric GUT theories [189, 190. Both electron and quark EDMs, and quark CDMs arise from one-loop diagrams with internal superparticles [186. Since electroweak gauginos are usually much lighter than gluinos, they typically dominate the one-loop diagrams. For a lightest superpartner mass of $100 \mathrm{GeV}$ (and assuming universality of the soft masses at a high scale) the experimental bound from ${ }^{205} \mathrm{Tl}$ on the electron EDM limits the phases to be $<0.01$ [187, 190. For the same set of parameters the bound from ${ }^{199} \mathrm{Hg}$ on the light quark CDM also coincidentally limits the phases to be $<0.01$ [187]. A similar constraint is obtained from the bound on the neutron EDM [186, 187, 190]. In assessing the reliability of these limits it is important to keep in mind that for both ${ }^{199} \mathrm{Hg}$ and the neutron there are large uncertainties introduced in the evaluation of the hadronic matrix elements of quark CDMs and EDMs (in the case of the neutron). In contrast, the bounds on the electron EDM from ${ }^{133} \mathrm{Cs}$ and ${ }^{205} \mathrm{Tl}$ are comparatively free from any atomic or nuclear uncertainties.

The smallness of the CP violating phases in supersymmetric models amounts to a mild naturalness problem. Various suppression mechanisms for the phases have been suggested, including CP conservation at the GUT scale [191], heavy superpartners [192], very light neutralinos [193], and dynamical relaxation of the phases 194.

As another example of a $\mathrm{CP}$ violating extension of the SM, multi-Higgs theories 
can possess phases in the Higgs potential which gives rise to EDMs at low energy. In this case since the Higgs-fermion coupling is proportional to the fermion mass, the dominant diagrams are two-loop, with an internal top quark or $W$ boson [195, 196]. Since here both the electron and quark EDMs and quark CDMs arise only at two loops, the bounds on the CP violating Higgs phases are not nearly as stringent as for supersymmetry. For a single light Higgs boson of mass $100 \mathrm{GeV}$, the current bounds on the electron EDM from ${ }^{133} \mathrm{Cs}$, and on the light quark CDM from ${ }^{199} \mathrm{Hg}$ and the neutron do not yet place limits on multi-Higgs theories. However, improvements in any of the bounds would begin to constrain the Higgs sector phases.

Finally, we consider the role of the $\mathrm{CP}$ violating $\mathrm{QCD}$ vacuum angle, $\bar{\theta}_{Q C D}$. Its contribution to strong sector $\mathrm{CP}$ violation is not suppressed by any large mass scale, making the EDM experiments very sensitive probes of non-zero $\bar{\theta}_{Q C D}$. The current bounds from ${ }^{199} \mathrm{Hg}$ and the neutron restrict the QCD vacuum angle to be $<$ few $\times 10^{-10}$, leading to the well known strong CP problem. With the atomic and molecular systems it is actually possible to distinguish $\bar{\theta}_{Q C D}$ from non-standard model CP violation. Since $\bar{\theta}_{Q C D}$ arises in the strong sector, it gives EDMs in the ratios indicated in the Strong column of Table 16. The main uncertainties in these ratios is from nuclear structure rather than hadronic matrix elements. Any deviation from this pattern would imply some component of non-standard model $\mathrm{CP}$ violation. In fact, given the current (extraordinary) bound on ${ }^{199} \mathrm{Hg}$, a positive measurement in ${ }^{133} \mathrm{Cs}$ above the level of $5 \times 10^{-27} \mathrm{e} \mathrm{cm}$, or in ${ }^{205} \mathrm{Tl}$ above the level of $5 \times 10^{-28} e \mathrm{~cm}$ would be a clear signal for (CP violating) physics beyond the standard model. This would be an important result of positive measurements in these atomic systems.

\section{Lepton Number Violation}

Individual lepton number is conserved in the SM with massless neutrinos. However, in many extensions of the SM, lepton flavor is violated by new physics which lies just above the electroweak scale[197]. The purely leptonic rare processes for which the best experimental bounds are available are given in Table 17 [198-203]. In the case of $\mu \rightarrow e$ conversion, we define $\mathrm{B} \equiv \sigma(\mu \mathrm{Ti} \rightarrow e \mathrm{Ti}) / \sigma(\mu \mathrm{Ti} \rightarrow$ capture $)$. Considerable improvements in some of the bounds are possible. At LAMPF the level $\mathrm{B}(\mu \rightarrow e \gamma)$ $\sim 6 \times 10^{-13}$ could be accessible [204, while at PSI B $(\mu \mathrm{Ti} \rightarrow e \mathrm{Ti}) \sim 3 \times 10^{-14}$ could be reached [205]. As discussed below these levels of precision would begin to probe much of the parameter space of supersymmetric GUT theories.

All the effective operators which contribute to the processes in Table 17 are effectively dimension-six and are suppressed by two powers of the scale characterizing lepton flavor violation. In most models the rate for $l \rightarrow l^{\prime} \gamma$ is proportional to $m_{l}^{2}$. The bound from $\mu \rightarrow e \gamma$ therefore typically gives more stringent limits on the microscopic physics than that from $\tau \rightarrow \mu \gamma$. In strongly coupled composite 


\begin{tabular}{|c|c|c|}
\hline \hline & Branching Ratio & Reference \\
\hline$\mu \rightarrow e \gamma$ & $<5 \times 10^{-11}$ & LAMPF-Crystal Box [198] \\
$\mu \rightarrow e e e$ & $<1 \times 10^{-12}$ & SINDRUM[199] \\
$\mu \mathrm{Ti} \rightarrow e \mathrm{Ti}$ & $<4 \times 10^{-12}$ & SINDRUM[200] \\
$\tau \rightarrow \mu \gamma$ & $<5 \times 10^{-6}$ & CLEO[201] \\
$Z \rightarrow e \mu$ & $<1.7 \times 10^{-6}$ & OPAL 202] \\
$Z \rightarrow e \tau$ & $<7.3 \times 10^{-6}$ & L3[203] \\
$Z \rightarrow \mu \tau$ & $<1.0 \times 10^{-5}$ & L3 203] \\
\hline \hline
\end{tabular}

Table 17: Experimental bounds on purely leptonic flavor violation.

models in which lepton flavor is not a good symmetry, the bound from $\mu \rightarrow e \gamma$ gives a lower limit on the composite scale of roughly $100 \mathrm{TeV}$.

Lepton flavor is not necessarily a symmetry of supersymmetric theories. The soft supersymmetry breaking terms which give the sleptons a large mass need not conserve flavor, and can lead to interesting levels for the processes listed in Table 17. The dominant diagrams arise at one-loop and involve mixing of internal sleptons. For superpartner masses of roughly $100 \mathrm{GeV}$, the bound from $\mu \rightarrow e \gamma$ limits the selectron-smuon mixing angle to be $\sin \theta_{\tilde{e} \tilde{\mu}}<3 \times 10^{-3}$. It has been emphasized recently that non-vanishing mixing between the sleptons is a generic feature of supersymmetric GUT theories which are unified below the Planck or compactification scale (and if supersymmetry breaking is transmitted by gravitational strength interactions] [206]. Above the GUT scale, quarks and leptons are unified within GUT multiplets. Renormalization group running between the Planck or compactification and GUT scales then mixes sleptons through CKM mixings. The large top-quark Yukawa coupling enhances this effect. For slepton masses in the few $\times 100 \mathrm{GeV}$ range, the branching ratio for $\mu \rightarrow e \gamma$ is within the range $10^{-13}-10^{-11}$ over much of the SUSY parameter space 206]. This is at or directly below the present experimental bound, and hence the future improvements [204, 205] therefore represent an important test of supersymmetric GUT theories.

Since the $\tau$ is the heaviest and least well-studied lepton, one might expect on rather general grounds that it is most likely to experience LFCI. In addition, due to the rather strong constraints arising from the decays $\mu \rightarrow e \gamma, \mu \rightarrow 3 e$, and $\mu-e$ conversion in atoms we might expect LFCI to be highly suppressed within the first two generations. LFCI involving the $\tau$ may appear in several ways. First, it is possible that the $\tau$ may have sizeable radiative decay modes, i.e., $\tau \rightarrow e \gamma, \mu \gamma$, or a significant decay to three lepton final states, i.e., $\tau \rightarrow 3 e, 3 \mu$, ee $\mu, \mu \mu e$. Secondly, the $\tau$ may have LFCI with the SM $Z$ so that the width for $Z \rightarrow \tau+e, \mu$ is of reasonable size. Within any given model it is likely that the rates for all of these processes are related so that, e.g., if LFCI of the $\tau$ appear in $Z$ decays, 
extensive detailed searches for LFCI in $\tau$ decays are warranted. As an example of this scenario, we consider a slightly more general version of the model of Eilam and Rizzo 197 wherein the existence of exotic fermions which mix with the ordinary SM leptons induces an off-diagonal coupling of the $Z$ to leptons. In such a model, the diagonal couplings of the leptons to the $Z$ differ little from the SM case

$$
\mathcal{L}_{S M}=\frac{g}{2 c_{w}} \bar{l} \gamma_{\mu}\left(v-a \gamma_{5}\right) l Z^{\mu}
$$

where as usual $v=-1 / 2+2 \sin ^{2} \theta_{w}$ and $a=-1 / 2$. The off-diagonal couplings can be written in a similar form

$$
\mathcal{L}_{L F C I}=\frac{g}{2 c_{w}} \bar{\tau} \gamma_{\mu}\left(v_{l}^{\prime}-a_{l}^{\prime} \gamma_{5}\right) l Z^{\mu}+\text { h.c. }
$$

where $l \neq \tau$ and with $v_{l}^{\prime}, a_{l}^{\prime}$ depending on the details of the model. In this notation, the branching fraction for the $\tau$ flavor-violating decay can be written as

$$
B\left(Z \rightarrow \tau^{-} l^{+}+\tau^{+} l^{-}\right)=2 B_{l} \frac{\left(v_{l}^{\prime}\right)^{2}+\left(a_{l}^{\prime}\right)^{2}}{v^{2}+a^{2}},
$$

with $B_{l}=0.034$ being the conventional SM leptonic branching fraction. Presently, the strongest $95 \% \mathrm{CL}$ bound on such decays are given in Table 17. These limits imply $\left(v_{\mu}^{\prime}\right)^{2}+\left(a_{\mu}^{\prime}\right)^{2} \leq 3.7 \times 10^{-5}$ and $\left(v_{e}^{\prime}\right)^{2}+\left(a_{e}^{\prime}\right)^{2} \leq 2.7 \times 10^{-5}$.

Of course, the existence of these $Z l \tau$ couplings directly induces the decay modes of $\tau$ into three leptons. For the processes $\tau \rightarrow e e \mu$ and $\tau \rightarrow \mu \mu e$ we obtain the branching fractions

$$
B=\left(v^{2}+a^{2}\right)\left[\left(v_{e, \mu}^{\prime}\right)^{2}+\left(a_{e, \mu}^{\prime}\right)^{2}\right] B_{\tau},
$$

where $B_{\tau}$ is the usual $\tau$ leptonic branching fraction. Using the L3 limits immediately implies that $B(\tau \rightarrow e e \mu) \leq 1.7 \times 10^{-6}$ and $B(\tau \rightarrow \mu \mu e) \leq 1.2 \times 10^{-6}$. The branching fractions for the $\tau \rightarrow 3 e, 3 \mu$ modes are a bit more complex due to the existence of identical particles in the final state; we obtain

$$
B=\frac{1}{2}\left[3\left(v^{2}+a^{2}\right)\left[\left(v_{e, \mu}^{\prime}\right)^{2}+\left(a_{e, \mu}^{\prime}\right)^{2}\right]+(2 v a)\left(2 v_{e, \mu}^{\prime} a_{e \mu}^{\prime}\right)\right] B_{\tau} .
$$

If the second term can be neglected, we then obtain bounds which are $50 \%$ larger than those stated above. A short calculation shows that the influence of the second term relative to the first is at most $5-6 \%$ and can occur with either sign.

\section{Double Beta Decay}

No-neutrino double beta decay is a low-energy process which tests mass scales beyond the reach of present accelerators. Thus, it is another promising way to search for physics beyond the SM. For it to occur would require not only that 
lepton number not be conserved but also that there be at least one additional piece of new physics. The latter might be the existence of electron neutrino mass, of a heavy Majorana neutrino which mixes with the electron neutrino, of righthanded currents, of a Goldstone boson (such as the Majoron), or of supersymmetric particles violating $R$ parity. Limits set on the lifetime for no-neutrino double beta decay therefore give corresponding restrictions on all these areas of possible new physics. Since this second-order weak process with potentially large phase space is so sensitive, these limits are generally better than can be set in any other existing experimental process. Should a positive effect ever be observed, however, most of these different potential sources of double beta decay are in principle indistinguishable experimentally from this process alone.

Since nuclei with even numbers of protons $(Z)$ and neutrons $(A-Z)$ are generally more stable than their odd-odd neighbors, such a nucleus can only decay via the emission of two electrons and two neutrinos to the next even-even nucleus, $(A, Z) \rightarrow(A, Z+2)+2 e^{-}+2 \bar{\nu}_{e}$, if it is energetically possible. Such very long-lived decays $\left(\sim 10^{20}\right.$ years $)$ have now been observed in several nuclei and serve as a test of nuclear matrix element calculations.

Of interest to particle physics are two potential lepton-number violating noneutrino decays. One is $(A, Z) \rightarrow(A, Z+2)+2 e^{-}+\chi^{0}$, by which limits have been set on neutrino-Majoron coupling, $\left\langle g_{\nu \chi}\right\rangle<10^{-4}$. The other is the more generally useful neutrinoless double beta decay, $(A, Z) \rightarrow(A, Z+2)+2 e^{-}$, which will be designated as $\beta \beta_{0 \nu}$. Even if neutrinos are Majorana particles, these decays would be highly suppressed because of the helicity reversal required in the neutrino exchange, however, the vastly increased phase space compared to the two-neutrino case makes these decays very sensitive probes of non-standard physics. Experimentally, the search for $\beta \beta_{0 \nu}$ is sensitive because one is looking for a spike in the summed electron energy spectrum. $\beta \beta_{0 \nu}$ is worth pursuing because it is an excellent method of experimentally determining whether neutrinos are Majorana or Dirac particles, and probably the only way one can ever hope to measure neutrino masses (as opposed to mass differences) in this very small mass range.

\subsection{Experimental Situation}

The current best limit on $\beta \beta_{0 \nu}$ is from experiments using kilograms of enriched ${ }^{76}$ Ge. The Heidelberg-Moscow group 207], which has the best bound at present, has reported a $90 \%$ C.L. he half-life limit of $5.6 \times 10^{24}$ years corresponding to an effective neutrino mass, $\left\langle m_{\nu}\right\rangle \approx\left|\sum_{i} \eta_{i} U_{e i}^{2} m_{\nu_{i}}\right|<0.65 \mathrm{eV}$, a value rather dependent on the nuclear matrix elements. The effective neutrino mass is a sum over those Majorana neutrino mass states, $m_{\nu_{i}}$, to which the electron neutrino couples via mixing matrix elements $U_{e i}$, and $\eta_{i}= \pm 1$ denotes the CP phase of the i-th neutrino.

While such bounds can provide interesting limits on new physics, no one has yet claimed a positive result. However, it is enough to intrigue experimentalists and 
to raise the question as to how much better one can do in limiting neutrino mass and other nonstandard physics by $\beta \beta_{0 \nu}$. This will be characterized by $\left\langle m_{\nu}\right\rangle$, but clearly these limits have many applications. The enriched Ge experiments, which now employ $6-8 \mathrm{~kg}$, have the potential to use about twice that amount of material. They could reach an order of magnitude better lifetime limit, or go from $\sim 1 \mathrm{eV}$ to $\sim 0.3 \mathrm{eV}$ in $\left\langle m_{\nu}\right\rangle$. A very large experiment, NEMO III, is being designed which uses sources separate from the detector, and a calorimeter with tracking, enabling it to reach a $\left\langle m_{\nu}\right\rangle$ limit $\sim 0.1 \mathrm{eV}$. Because the resolution is poor $\left(27 \%\right.$ at the ${ }^{100} \mathrm{Mo}$ endpoint of $3030 \mathrm{keV}$ ), this device would have difficulty establishing a positive effect, but it does have the potential of improving the present bounds.

Two other isotopes would be competitive and indeed hold promise of going to still smaller values of $\left\langle m_{\nu}\right\rangle$, but they are only at the test phase. One is ${ }^{136} \mathrm{Xe}$, which has been used successfully in a gas Time Projection Chamber, but liquid Xe is probably required, along with the use of scintillation and/or ionization, to get sufficient mass. Scaling up to ton quantities is possible. The other isotope is ${ }^{150} \mathrm{Nd}$, which has $\sim 70$ times the sensitivity of ${ }^{76} \mathrm{Ge}$. The best form of a detector here, is one in which the source and detector are the same. This use of ${ }^{150} \mathrm{Nd}$ has not been possible until recently with the advent of cryogenic bolometers. Indications are that one could have $1 \mathrm{keV}$ resolution (three times better than Ge ionization detectors) at the endpoint of $3467 \mathrm{keV}$, which is higher in energy than all natural $\beta$ and $\gamma$ radioactivity, thus reducing background problems. The UCSB, CfPA, Stanford, Baksan group is testing this approach and will probably add a $\sim 0.1 \mathrm{~kg}$ ${ }^{150} \mathrm{Nd}$ detector to their dark matter experiment.

\subsection{Neutrinoless Double Beta Decay and Physics Beyond The Standard Model}

The bounds obtained by the Heidelberg-Moscow collaboration 207 imply strong upper limits on the strength of lepton number violating interactions. Since the standard electroweak model conserves $B-L$ quantum number, the above upper limits would provide important information on new physics scenarios beyond the standard model that involve lepton number violation. There exist several well motivated scenarios of new physics that fall into this category. The models that we will discuss here are: (i) the left-right symmetric models of weak interaction with the see-saw mechanism for neutrino masses 208, 209], (ii) the MSSM where without the additional assumption of R-parity conservation one has both lepton and baryon number violating terms [14, and (iii) composite models for leptons.

To see how one extracts constraints on new physics from the observed lower limit on the lifetime for $\beta \beta_{0 \nu}$ process, let us parameterize the amplitude for this process as

$$
A_{\beta \beta} \simeq G_{F}^{2}\left(\mu_{\beta \beta}^{2}\right)
$$


where we have hidden all nuclear physics effects in the effective mass $\mu_{\beta \beta}$. The width for the decay can then be written as

$$
\Gamma_{\beta \beta} \simeq \frac{Q^{5}\left|A_{\beta \beta}\right|^{2}}{60 \pi^{3}}
$$

Here, $Q$ is the available energy for the two electrons. To get a feeling for the upper limit on $\mu_{\beta \beta}$, note that the present ${ }^{76} \mathrm{Ge}$ experiment 207 implies that $\Gamma_{\beta \beta} \leq$ $3.477 \times 10^{-57} \mathrm{GeV}$; using for a rough estimate $Q \simeq 2.3 \mathrm{MeV}$, we find that $\mu_{\beta \beta} \leq$ $10^{-5} \mathrm{GeV}$. We will now estimate the parameter $\mu_{\beta \beta}$ for various extensions of the SM and translate this upper limit into constraints on the parameters of the model.

Let us first consider the classic contribution of the Majorana neutrino mass to this process 210. In this case, one gets $\mu_{\beta \beta}^{2}=\left\langle m_{\nu}\right\rangle p_{F} f_{N}\left(p_{F}\right.$ is the Fermi momentum in the nucleus $\approx 50 \mathrm{MeV}$, and $\left\langle m_{\nu}\right\rangle$ is as defined above). Barring nuclear uncertainties 211 ( hidden in the factor $f_{N}$ ), detailed calculations lead to the upper bound $\left\langle m_{\nu}\right\rangle \leq .65 \mathrm{eV}$.

\section{- Left-right symmetric model}

There are four new contributions to the $\beta \beta_{0 \nu}$ process in the LRM in addition to the neutrino mass diagram just discussed: (i) the first one arises from the exchange of heavy Majorana right handed neutrinos $\left(N_{R}\right)$ and the right handed $W$ bosons (see Fig. 23); (ii) that arising from a $W_{L}-W_{R}$ exchange and therefore necessarily involving the mixing between the light and heavy neutrinos; (iii) exchange of a doubly charged Higgs boson as shown in Fig. 24; (iv) vector scalar exchange 212 involving a singly charged Higgs boson and the left-handed $W_{L}$ boson . The present upper bound on the $\beta \beta_{0 \nu}$ amplitude then leads to restrictions on the parameters of the model involved in these various graphs. We summarize the constraints obtained on these separate contributions below, where we assume that the strength of the right-handed interactions and the right-handed CKM matrix are equal to their left-handed counterparts (i.e., $g_{R}=g_{L}$ and $V_{i j}^{R}=V_{i j}^{L}$ ).

Heavy $N_{R}$ exchange

The effective mass parameter in this case can be written as (where $\zeta$ denotes the amount of heavy-light neutrino mixing)

$$
\mu_{\beta \beta}^{2} \simeq\left(p_{e f f}^{3}\right)\left(\frac{M_{W_{L}}^{4}}{M_{W_{R}}^{4}}+\zeta^{2}\right) \frac{1}{m_{N}} .
$$

Here $p_{\text {eff }}$ is an effective momentum chosen to have a value of $50 \mathrm{MeV}$. The present limits on the neutrinoless double beta decay lifetime then impose a correlated constraint on the parameters $M_{W_{R}}$ and $m_{N}$ [213].

Light-heavy neutrino mixing contribution: 
Figure 23: Heavy right handed neutrino contribution to $\beta \beta_{0 \nu}$ in the LRM.

Figure 24: Contribution of the doubly charged Higgs boson in the LRM. 
In this case, one finds $\mu_{\beta \beta}^{2} \simeq \zeta\left(M_{W_{L}}^{2} / M_{W_{R}}^{2}\right)\left(p_{e f f}^{2}\right)$. This leads to the correlated constraint on $\zeta$ and $M_{W_{R}}$ shown in Fig. 25. If we combine the theoretical constraints of vacuum stability then, the present ${ }^{76} \mathrm{Ge}$ data provides a lower limit on the masses of the right handed neutrino $\left(N_{e}\right)$ and the $W_{R}$ of $1 \mathrm{TeV}$, which is a rather stringent constraint. The limits on $\zeta$ on the other hand are not more restrictive than what would be expected from the structure of the theory. We have of course assumed that the leptonic mixing angles are small, so that there is no cancellation between the parameters.

Figure 25: Bounds on the light and heavy neutrino mixing parameter in the LRM from ${ }^{76}$ Ge data.

\section{Higgs contributions:}

The two types of Higgs induced contributions to $\beta \beta_{0 \nu}$ decay indicated above lead to the following expressions for $\mu_{\beta \beta}$. The one arising from the coupling of the doubly charged Higgs boson to electrons leads to

$$
\mu_{\beta \beta_{H}}^{2}=\left(\frac{f_{11}}{M_{\Delta}^{2}}\right) 2^{7 / 4} G_{F}^{3 / 2}\left(\frac{M_{W_{L}}}{M_{W_{R}}}\right)^{3} .
$$

The present ${ }^{76} \mathrm{Ge}$ data implies that (assuming $M_{W_{R}} \geq 1 \mathrm{TeV}$ ) $M_{\Delta^{++}} \geq \sqrt{f_{11}} 80$ $\mathrm{GeV}$. The second contribution arises from the mixing amongst the charged Higgs fields after the full gauge symmetry is broken down to $U(1)_{\mathrm{em}}$. Denoting this mixing term by the angle $\theta$, one can get in this case,

$$
\mu_{\beta \beta_{H}^{\prime}}^{2} \simeq \frac{h_{u} f_{11} \sin 2 \theta p_{e f f}^{2}}{4 \sqrt{2} G_{F} M_{H^{+}}^{2}},
$$


where we have assumed that the $H^{+}$is the lighter of the two Higgs fields, and $f_{11}$ and $h_{u}$ are Yukawa couplings associated with the triplet and bi-doublet Higgs fields, respectively. One then finds 212 $h_{u} f_{11} \sin 2 \theta \leq 6 \times 10^{-9}\left(M_{H^{+}} / 100 \mathrm{GeV}\right)^{2}$, which is quite a stringent constraint on the parameters of the theory. To appreciate this somewhat more, we point out that one expects $h_{u} \approx m_{u} / M_{W} \approx 5 \times 10^{-5}$ in which case, we get an upper limit for the coupling of the Higgs triplets to leptons $f_{11} \sin 2 \theta \leq 10^{-4}$ (for $m_{H^{+}}=100 \mathrm{GeV}$ ). Taking a reasonable choice of $\theta \sim M_{W_{L}} / M_{W_{R}} \sim 10^{-1}$ would correspond to a limit $f_{11} \leq 10^{-3}$. Bounds on this parameter from an analysis 214 of Bhabha scattering are only or order $\sim 0.2$ for the same value of the Higgs mass.

\section{- MSSM with R-parity violation}

The next class of theories we will consider is the case of supersymmetry with $\mathrm{R}$-parity violation 14. The $\mathrm{R}$-violating part of the potential is given in Section 2. In order to maintain proton stability we set $\lambda^{\prime \prime}=0$ in the superpotential of Eq. (3). The first contribution to $\beta \beta_{0 \nu}$ decay is dominantly mediated by heavy gluino exchange[215] as shown in Fig. 26. Detailed evaluation of the nuclear matrix element for this class of models 216 has led to the following bound on the R-violating parameter,

$$
\lambda_{111}^{\prime} \leq 3.9 \times 10^{-4}\left(\frac{m_{\tilde{q}}}{100 G e V}\right)^{2}\left(\frac{m_{\tilde{g}}}{100 G e V}\right)^{1 / 2} .
$$

The second class of contribution is presented in Fig. 27. This leads to a contribution to $\mu_{\beta \beta}^{2}$ given by

$$
\mu_{\beta \beta}^{2} \simeq\left(\frac{\left(\lambda_{113}^{\prime} \lambda_{131}^{\prime}\right)}{2 \sqrt{2} G_{F} M_{\tilde{b}}^{2}}\right)\left(\frac{m_{b}}{M_{\tilde{b}^{c}}^{2}}\right)\left(\mu \tan \beta+A_{b} m_{0}\right)\left(p_{e f f}^{3}\right) .
$$

Here $A_{b}, m_{0}$ are supersymmetry breaking parameters, and $\mu$ is the supersymmetric mass of the Higgs bosons, as discussed in Section 2. For the choice of all squark masses, as well as $\mu$ and the SUSY breaking mass parameters, being of order of 100 $\mathrm{GeV}, A_{b}=1, \tan \beta=1$, the following bound on R-violating couplings is obtained,

$$
\lambda_{113}^{\prime} \lambda_{131}^{\prime} \leq 3 \times 10^{-8} .
$$

If the exchanged scalar particles in Fig. 27 are the $\tilde{s}-\tilde{s}^{c}$ pair, one obtains the constraint

$$
\lambda_{121}^{\prime} \lambda_{112} \leq 1 \times 10^{-6}
$$

\section{- Limits on the scale of lepton compositeness}

If the quarks and leptons are composite particles, it is natural to expect excited leptons which will interact with the electron via some effective interaction involving 
Figure 26: Gluino mediated contribution in MSSM with R-parity violation.

Figure 27: Vector-scalar contribution in MSSM with R-parity violation. 
the $W_{L}$ boson. If the excited neutrino is a majorana particle, then there will be contributions to $\beta \beta_{0 \nu}$ decay mediated by the excited neutrinos $\left(\nu^{*}\right)$. The effective interaction responsible for this is obtained from the primordial interaction

$$
H_{e f f}^{\nu^{*}}=g \frac{\lambda_{W}^{\left(\nu^{*}\right)}}{2 m_{\nu^{*}}} \bar{e} \sigma^{\mu \nu}\left(\eta_{L}^{*}\left(1-\gamma_{5}\right)+\eta_{R}^{*}\left(1+\gamma_{5}\right)\right) \nu^{*} W_{\mu \nu}+\text { h.c. }
$$

Here $\mathrm{L}$ and $\mathrm{R}$ denote the left and right chirality states. This has been studied in detail in two recent papers 217] and leads to the bound (taking $\left|\eta_{L}^{*}\right|^{2}+\left|\eta_{R}^{*}\right|^{2}=1$ with $\eta_{L}^{*} \eta_{R}^{*}=0$, and assuming that the excited neutrino is a Majorana particle)

$$
m_{\nu^{*}} \geq 5.9 \times 10^{4} \mathrm{TeV}
$$

for $\lambda_{W}^{\left(\nu^{*}\right)} \geq 1$. Here, the compositeness scale has been set to be the mass of the excited neutrino. However, this yields a rather stringent bound on the compositeness scale!

\section{- Models with heavy sterile neutrinos}

The see-saw mechanism for understanding small neutrino masses always requires the introduction of heavy neutral sterile fermions. If there is a single sterile neutrino within the standard gauge model, then constraints from the see-saw mechanism suppress the heavy sterile contribution to the $\beta \beta_{0 \nu}$ process. Since the heavy sterile sector is largely unknown, a possibility to consider is to have two heavy sterile leptons which participate in a $3 \times 3$ see-saw with the light neutrino to make $m_{\nu}$ small. The analog of the mixing parameter $\zeta$ is then not constrained to be small 218 by the see-saw considerations and also a larger range of masses for the heavy sterile particles are then admissible. Such models are however subject to a variety of cosmological and astrophysical constraints. These constraints have been analyzed in detail in 218 and it is found that there is a large range of the parameter space for the sterile particles which can be probed by the ongoing neutrinoless double beta experiments.

\section{Summary}

In conclusion, we have shown that a large number of processes are influenced by the virtual effects of new physics in higher order interactions and thus have tremendous power in probing physics beyond the SM. This attack on the search for new physics is important as it can probe higher energy scales and it provides a complementary search reach to direct production at colliders. The drawbacks are, however, that (i) simultaneous virtual contributions of many new particles have the potential to cancel each other's effects, (ii) not all models have large indirect effects, and (iii) an enormous amount of very precise data must be gathered. Considering our lack of knowledge on what lies beyond the Standard Model, we stress the importance 
of searching for effects of new physics via every possible means and cross-checking any positive signal in the largest number of processes available. However, these indirect probes should not be recommended as providing a complete substitute for continuing our search new physics at ever-higher energy colliders.

\section{Acknowledgements}

We would like to thank G. Burdman, S. Chu, M. Cooper, D. Demille, N. Fortson, E. Golowich, A. Grant, L. Hall, D. Iteinzen, S. Lamoreaux, W. Marciano, S. Pakvasa, J. Rosner, and E. Thorndike for useful discussions.

\section{References}

[1] A. Djouadi, J.N. Ng, and T.G. Rizzo, these proceedings.

[2] A. Acker and S. Pakvasa, Mod. Phys. Lett. A7, 1219 (1992).

[3] L. Rolandi, H. Dijkstra, D. Strickland, and G. Wilson, representing the ALEPH, DELPHI, L3, and OPAL Collaborations, Joint Seminar on the First Results from LEP 1.5, CERN, Dec. 12, 1995.

[4] C.T. Hill and E.A. Paschos, Phys. Lett. B241, 96 (1990).

[5] L. Montanet et al., Particle Data Group, Phys. Rev. D50, 1173 (1994), and 1995 off-year partial update for the 1996 edition available on the PDG WWW pages (URL:http://pdg.lbl.gov/).

[6] For a review of $E_{6}$ theories, see, J.L. Hewett and T.G. Rizzo, Phys. Rep. 183, 193 (1989).

[7] V. Barger, M.S. Berger, and R.J.N. Phillips, Phys. Rev. D52, 1663 (1995); D. London, in Precision Tests of the Standard Model, ed. P. Langacker, (World Scientific, 1993); D. London and P. Langacker, Phys. Rev. D38, 886 (1988); B.A. Campbell et al., Int. J. Mod. Phys. A2, 831 (1987).

[8] J. Maalampi and M. Roos, Phys. Rep. 186, 53 (1990); I. Montvay, Phys. Lett. B205, 315 (1988); C. Csaki and F. Csikor, Phys. Lett. B309, 103 (1993).

[9] J.F. Gunion et al., The Higgs Hunter's Guide, (Addison-Wesley, Redwood City, CA 1990).

[10] V. Barger, J.L. Hewett, and R.J.N. Phillips, Phys. Rev. D41, 3421 (1990); A. Buras et al., Nucl. Phys. B337, 284 (1990); J.F. Gunion and B. Grzadkowski, Phys. Lett. B243, 301 (1990). 
[11] S. Weinberg, Phys. Rev. Lett. 37, 657 (1976); P. Krawczyk and S. Pokorski, Nucl. Phys. B364, 10 (1991); Y. Grossman and Y. Nir, Phys. Lett. B313, 126 (1993); Y. Grossman, Nucl. Phys. B426, 355 (1994).

[12] S. Pakvasa and H. Sugawara, Phys. Lett. 73B, 61 (1978); S. Pakvasa, H. Sugawara, and Y. Yamanaka, Phys. Rev. D25, 1895 (1982); T.P. Cheng and M. Sher, Phys. Rev. D35, 3484 (1987); L.J. Hall and S. Weinberg, Phys. Rev. D48, 979 (1993); A. Antaramian, L.J. Hall, and A. Rasin, Phys. Rev. Lett. 69, 1871 (1992).

[13] H. Baer, et al., these proceedings; X. Tata, lectures given at TASI 1995, Univ. of Colorado, June 1995, hep-ph/9510287, and references therein.

[14] L. Hall and M. Suzuki, Nucl. Phys. B231, 419 (1984); S. Dimopoulos and L.J. Hall, Phys. Lett. B207, 210 (1987); V. Barger, G.F. Guidice and T. Han, Phys. Rev. D40, 2987 (1989); C.E. Carlson, P. Roy, and M. Sher, Phys. Lett. B357, 99 (1995); G. Bhattacharyya and D. Choudhury, Mod. Phys. Lett. A10, 1699 (1995); G. Bhattacharyya, D. Choudhury, and K. Sridhar, Phys. Lett. B355, 193 (1995); G. Bhattacharyya, J. Ellis, and K. Sridhar, Mod. Phys. Lett. A10, 1583 (1995).

[15] For a review and original references, see R.N. Mohapatra, Unification and Supersymmetry, (Springer, New York, 1986).

[16] A. Jodiddo et al., Phys. Rev. D34, 1967 (1986), and Phys. Rev. D37, 237 (1988); J. Imazoto et al., Phys. Rev. Lett. 69, 877 (1992); A. Sirlin, Phys. Rev. Lett. 72, 1786 (1994).

[17] E. Ma, Phys. Rev. D36, 274 (1987), and Mod. Phys. Lett. A3, 319 (1988); K.S. Babu et al., Phys. Rev. D36, 878 (1987); V. Barger and K. Whisnant, Int. J. Mod. Phys. A3, 879 (1988); J.F. Gunion et al., Int. J. Mod. Phys. A2, 118 (1987).

[18] S. Davidson, D. Bailey, and B.A. Campbell, Z. Phys. C61, 613 (1994); M. Leurer, Phys. Rev. D50, 536 (1994), and D49, 333 (1994).

[19] H. Aihara et al., these proceedings. See also, K. Hagiwara et al., Nucl. Phys. B282, 253 (1987).

[20] M. E. Peskin and T. Takeuchi, Phys. Rev. Lett. 65, 964 (1990); Phys. Rev. D46, 381 (1992).

[21] I. Maksymyk, C.P. Burgess, and D. London, Phys. Rev. D50, 529 (1994); C.P. Burgess, hep-ph/9411257, to appear in the Proceedings of the Third Workshop on High Energy Physics, Madras, India, January 1994. 
[22] T. Takeuchi, A.K. Grant, and J.L. Rosner, in Proceedings of the DPF'94 Meeting, Albuquerque, NM, Aug. 1994, edited by S. Seidel (World Scientific, Singapore, 1995).

[23] S. Weinberg, Phys. Rev. Lett. 19, 1264 (1967); A. Salam, in Elementary Particle Theory, ed. N. Svartholm (Almquist and Wiksells, Stockholm, 1968).

[24] LEP Electroweak Working Group, P. Antilogus et al., Report No. LEPEWWG/95-02 (August 1995), LEP Electroweak Heavy Flavor Working Group and SLD Heavy Flavor Group, D. Abbaneo et al., Report No. LEPHF/95-02, SLD Physics Note 39 (July 1995).

[25] J. Alitti et al., UA2 Collaboration, Phys. Lett. B241, 150 (1990); F. Abe et al., CDF Collaboration, Phys. Rev. Lett. 62, 1005 (1989), 75, 11 (1985), and Phys. Rev. D52, 4784 (1995); C.K. Jung, D0 Collaboration, in the Proceedings of the $27^{\text {th }}$ International Conference on High Energy Physics, Glasgow, Scotland, July 1994, ed. P.J. Bussey and I.G. Knowles (Institute of Physics, Bristol 1995) Vol. 2, p.429.

[26] J.V. Allaby et al., CHARM Collaboration, Z. Phys. C36, 611 (1987); A. Blondel et al., CDHS Collaboration, Z. Phys. C45, 361 (1990); C.G. Arroyo et al., CCFR Collaboration, Phys. Rev. Lett. 72, 3452 (1994).

[27] P. Langacker and J. Erler, Phys. Rev. D50, 1304 (1994).

[28] J. Dorenbosch et al., CHARM I Collaboration, Z. Phys. C41, 567 (1989); L.A. Ahrens, BNL E734, Phys. Rev. D41, 3297 (1990); P. Vilain et al., CHARM II Collaboration, Phys. Lett. B281, 159 (1992).

[29] J. Rosner, Univ. of Chicago Report EFI-95-41, hep-ph/9507375.

[30] F. Abe et al., CDF Collaboration, Phys. Rev. Lett. 73, 225 (1994), 74, 2626 (1995), and Phys. Rev. D50, 2966 (1995); S. Abachi et al., D0 Collaboration, Phys. Rev. Lett. 74, 2632 (1995).

[31] The ZFITTER package: D. Bardin et al., Z. Phys. C44, 493 (1989); Nucl. Phys. B351, 1 (1991); Phys. Lett. B255, 290 (1991); CERN report CERNTH-6443/92, 1992.

[32] W.J. Marciano and A. Sirlin, Phys. Rev. D22, 2695 (1980), D27, 552 (1983), and D29, 75 (1984); S. Sarantakos, A. Sirlin, and W.J. Marciano, Nucl. Phys. B217, 84 (1983); G. Degrassi, A. Sirlin, and W.J. Marciano, Phys. Rev. D39, 287 (1989). 
[33] For a discussion on recent attempts to improve the determination of $\alpha\left(M_{Z}\right)$, see, T. Takeuchi, in the Proceedings of the International Symposium on Vector Boson Self-Interactions, Los Angeles, CA, February 1995, ed. U. Baur, S. Errede, and T. Müller (AIP Press, 1995); and M.L. Schwarz, SLAC-PUB-957001, hep-ph/9509248.

[34] S. Bethke, in Proceedings of the Workshop on Physics and Experiments with Linear $e^{+} e^{-}$Colliders, Waikoloa, Hawaii, April 1993, ed. F.A. Harris et al., (World Scientific, Singapore 1993) p.687.

[35] C.H. Llewellyn Smith, Nucl. Phys. B228, 205 (1983).

[36] M.C. Noecker, B.P. Masterson, and C.E. Wieman, Phys. Rev. Lett. 61, 310 (1988).

[37] M.J.D. Macpherson, K.P. Zetie, R.B. Warrington, D.N. Stacey, and J.P. Hoare, Phys. Rev. Lett. 67, 2784 (1991).

[38] D.M. Meekhof, P.A. Vetter, P.K. Majumder, S.K. Lamoreaux, and E.N. Fortson, Phys. Rev. Lett. 71, 3442 (1993).

[39] P.A. Vetter, D.M. Meekhof, P.K. Majumder, S.K. Lamoreaux, and E.N. Fortson, Phys. Rev. Lett. 74, 2658 (1995).

[40] N.H. Edwards, S.J. Phipp, P.E.G. Baird, and S. Nakayama, Phys. Rev. Lett. 74, 2654 (1995).

[41] M.A. Bouchiat and C.C. Bouchiat, Phys. Lett. 48B, 111 (1974).

[42] V.A. Dzuba, V.V. Flambaum, and O.P. Sushkov, Phys. Lett. A141, 147 (1)989 ; S.A. Blundell, W.R. Johnson, and J. Sapirstein, Phys. Rev. Lett. 65, 1411 (1990), and Phys. Rev. D45, 1602 (1992).

[43] D.C. Kennedy and B.W. Lynn, Nucl. Phys. B322, 1 (1989).

[44] S. Bertolini and A. Sirlin, Phys. Lett. B257, 179 (1991); E. Gates and J. Terning, Phys. Rev. Lett. 67, 1840 (1991); E. Ma and P. Roy, Phys. Rev. Lett. 68, 2879 (1993); B.A. Kniehl and H.-G. Kohrs, Phys. Rev. D48, 225 (1993).

[45] T. Appelquist and J. Terning, Phys. Lett. B315, 139 (1993).

[46] L. Lavoura and J.P. Silva, Phys. Rev. D47, 2046 (1993); N. Maekawa, Prog. Theor. Phys. 93, 919 (1995), and Phys. Rev. D52, 1684 (1995). 
[47] A. Denner, R.J. Guth, and J.H. Kühn, Phys. Lett. B240, 438 (1990); M.J. Dugan and L. Randall, Phys. Lett. B264, 154 (1991); H. Georgi, Nucl. Phys. B363, 301 (1991); C.D. Froggatt, R.G. Moorhouse, and I.G. Knowles, Phys. Rev. D45, 2471 (1992); L. Lavoura and L.-F. Li, Phys. Rev. D48, 234 (1993).

[48] J..L. Rosner, M.P. Worah, and T. Takeuchi, Phys. Rev. D49, 1363 (1994).

[49] L. Lavoura and L.-F. Li, Phys. Rev. D49, 1409 (1994).

[50] S. Fleming and I. Maksymyk, Univ. of Texas Report UTTG-04-95, hep ph/9504272 (April 1995).

[51] See the talk given by K. Hagiwara at the 17th International Symposium on Lepton-Photon Interactions, Beijing, China, August 1995, hep-ph/9512425.

[52] G. Altarelli, R. Barbieri, and F. Caravaglios, Nucl. Phys. B405, 3 (1993), and Phys. Lett. B314, 357 (1993); A. Blondel, A. Djouadi, and C. Verzagnassi, Phys. Lett. B293, 253 (1992); A. Blondel and C. Verzagnassi, Phys. Lett. B311, 346 (1993); D. Comelli, C. Verzagnassi, and F.M. Renard, Phys. Rev. D50, 3076 (1994).

[53] P. Bamert, McGill Univ. Report McGill/95-64, hep-ph/9512445.

[54] M. Boulware, D. Finnell, Phys. Rev. D44, 91 (2054).

[55] J. Wells, G. Kane, SLAC-PUB-95-7038, hep-ph/9510372.

[56] A. Djouadi, P. Zerwas, and J. Zunft, Phys. Lett. B259, 175 (1991).

[57] Y. Grossman, H. Haber, Y. Nir, Phys. Lett. B357, 630 (1995); W.-S. Hou, Phys. Rev. D48, 2342 (1993); P. Krawczyk and S. Pokorski, Phys. Rev. Lett. 60, 182 (1988); J.L. Hewett, in Proceedings of the 1992 DPF Meeting, Fermilab, ed. J. Yoh (World Scientific, 1993).

[58] E. Ma, D. Ng, TRIUMF Report TRI-PP-95-55, hep-ph/9508338.

[59] J. Sola, Barcelona Report UAB-FT-370, hep-ph/9508339.

[60] J. Ellis, J.L. Lopez, and D.V. Nanopoulos, CERN Report CERN-TH/95-314, hep-ph/9512288.

[61] G. Kane, R. Stuart, J. Wells, Phys. Lett. B354, 350 (1995).

[62] P. Chankowski, S. Pokorski, in Proceedings of the 4th International Conference on Physics Beyond the Standard Model, Lake Tahoe, CA, December 1994, ed. J. Gunion et al., (World Scientific, Singapore 1995). 
[63] D. Garcia, J. Sola, Phys. Lett. B354, 335 (1995), and B357, 349 (1995).

[64] A. Dabelstein, W. Hollik, W. Mosle, hep-ph/9506251.

[65] For a review and list of references, see M. Drees and S. Martin, these proceedings.

[66] J. Wells, C. Kolda, G. Kane, Phys. Lett. B338, 219 (1994).

[67] M. Carena, C. Wagner, Nucl. Phys. B452, 45 (1995).

[68] S. Dimopoulos and L. Susskind, Nucl. Phys. B155, 237 (1979); E. Eichten and K. Lane, Phys. Lett. 90B, 125 (1980).

[69] S. Weinberg, Phys. Rev. D13, 974 (1976), and D19 1277 (1979); L. Susskind, Phys. Rev. D20 2619 (1979).

[70] R.S. Chivukula, S.B. Selipsky, and E.H. Simmons, Phys. Rev. Lett. 69575 (1992).

[71] A. Manohar and H. Georgi, Nucl. Phys. B234, 189 (1984).

[72] B. Holdom, Phys. Lett. B105, 301 (1985); K. Yamawaki, M. Bando and K. Matumoto, Phys. Rev. Lett. 56, 1335 (1986); V.A. Miransky, Nuovo Cim. A, 90 (1985); T. Appelquist, D. Karabali, and L.C.R. Wijewardhana, Phys. Rev. D35, 389 (1987); T. Appelquist and L.C.R Wijewardhana, Phys. Rev. D35, 774 (1987), and Phys. Rev. D36, 568 (1987).

[73] R.S. Chivukula, E. Gates, E.H. Simmons, and J. Terning, Phys. Lett. B311, 383 (1993).

[74] T. Appelquist, M. Einhorn, T. Takeuchi, and L.C.R. Wijewardhana, Phys. Lett. B220, 223 (1989); V.A. Miransky and K. Yamawaki, Mod. Phys. Lett. A4, 129 (1989); K. Matumoto Prog. Theor. Phys. Lett. 81, 277 (1989).

[75] R.S. Chivukula, K. Lane, and A.G. Cohen, Nucl. Phys. B343, 554 (1990); T. Appelquist, J. Terning, and L. Wijewardhana, Phys. Rev. D44, 871 (1991); E.H. Simmons, Nucl. Phys. B312, 253 (1989).

[76] N. Evans, Phys. Lett. B331, 378 (1994); C.D. Carone, E.H. Simmons, and Y. Su. Phys. Lett. B344, 287 (1995).

[77] T. Yoshikawa, Mod. Phys. Lett A10, 1601 (1995); G.-H. Wu, Phys. Rev. Lett. 74, 4137 (1995); K. Hagiwara and N. Kitazawa, hep-ph/9504332; C.-X. Yue, Y.P. Kuang, G.-R. Lu, L.-D. Wan, hep-ph/9506480. 
[78] R.S. Chivukula, E.H. Simmons, and J. Terning, Phys. Lett. B331, 383 (1994). R.S. Chivukula, E.H. Simmons, and J. Terning, hep-ph/9506427.

[79] A. Grifols and A. Mendez, Phys. Lett. B255, 611 (1991), and erratum Phys. Lett. B259, 512 (1991); B. Ananthanarayan and S.D. Rindani Phys. Rev. Lett. 73, 1215 (1994); G. Köpp et al., Z. Phys. C65, 545 (1995); F. del Aguila and M. Sher, Phys. Lett. B252, 116 (1990); R. Escribano and E. Masso, Phys. Lett. B301, 419 (1993), and Nucl. Phys. 429, 19 (1994); W. Bernreuther, O. Nachtmann and P. Overmann, Phys. Rev. D48, 78 (1993); G. Couture, Phys. Lett. B305, 306 (1993), and Phys. Lett. B272, 404 (1991); G. Domokos et al., Phys. Rev. D32, 247 (1985); J. Reid, M. Samuel, K.A. Milton and T.G. Rizzo, Phys. Rev. D30, 245 (1984). See also, P.D. Acton et al., OPAL Collaboration, Phys. Lett. B281, 305 (1992); D. Buskulic et al., ALEPH Collaboration, Phys. Lett. B297, 459 (1992).

[80] For an overview, see R. Frey, talk presented at the Future $e^{+} e-$ Collider Physics Study Group Meeting, Estes Park, Colorado, June 21-23, 1995. See also, G. Kane, G.A. Ladinsky and C.P. Yuan, Phys. Rev. D45, 124 (1992); C.P. Yuan, Phys. Rev. D45, 782 (1992); D. Atwood, A. Aeppli and A. Soni, Phys. Rev. Lett. 69, 2754 (1992); D. Atwood, A. Kagan and T.G. Rizzo, Phys. Rev. D52, 6264 (1995); M. Peskin, in Proceedings of the Second International Workshop on Physics and Experiments at Linear $e^{+} e^{-}$Collider, Waikoloa, HI, April 1993, ed. F.A. Harris et al., (World Scientific, 1994); M. Peskin and C.R. Schmidt, in Proceedings of the First Workshop on Linear Colliders, Saariselkä, Finland, September 1991 (World Scientific 1992); P. Zerwas, ibid.; W. Bernreuther et al., in Proceedings of the Workshop on $e^{+} e^{-}$Collisions at $500 \mathrm{GeV}$, The Physics Potential, (DESY, Hamburg) ed. by P. Igo-Kemenes and J.H. Kühn, 1992; A. Djouadi, ENSLAPP-A-365-92 (1992); T.G. Rizzo, Phys. Rev. D50, 4478 (1994); M. Frigeni and R. Rattazzi, Phys. Lett. B269, 412 (1991); R.D. Peccei, S. Persis and X. Zhang, Nucl. Phys. B349, 305 (1991); D.O. Carlson, E. Malkawi and C.-P. Yuan, Phys. Lett. B337, 145 (1994).

[81] T.G. Rizzo, Phys. Rev. D51, 3811 (1995), and SLAC-PUB-95-6914, 1995.

[82] G. Altarelli, R. Barbieri, and S. Jadach, Nucl. Phys. B331, 541 (1990), and (erratum) ibid., B376, 444 (1992); G. Altarelli, R. Barbierei, and F. Caravaglios, ibid., B405, 3 (1993); G. Altarelli et al., Phys. Lett. B318, 139 (1993).

[83] T.G. Rizzo, Phys. Rev. D50, 2256 (1994), and D48, 5286 (1993).

[84] For a review see, P. Langacker, in Precision Tests of the Standard Model, ed. P. Langacker (World Scientific, Singapore 1995). 
[85] See, M. Cvetic and S. Godfrey, these proceedings.

[86] W.J. Marciano and J.L. Rosner, Phys. Rev. Lett. 65, 2963 (1990), and (erratum) 68, 898(E) (1992); K.T. Mahanthappa and P.K. Mohapatra, Phys. Rev. D43, 3093 (1991); T.G. Rizzo, University of Wisconsin Report MAD/PH/604 (1990).

[87] C.P. Burgess, S. Godfrey, H. König, D. London and I. Maksymyk, Phys. Rev. D49, 6115 (1994).

[88] G. Altarelli and R. Barbieri, Phys. Lett. B253, 161 (1991).

[89] D.C. Kennedy and P. Langacker, Phys. Rev. Lett. 65, 90 (2967); and see Marciano and Rosner in Ref. [86].

[90] J. Bailey, et al., Nucl. Phys. B150, 1 (1979).

[91] T. Kinoshita and W. Marciano, in Quantum Electrodynamics, ed. by T. Kinoshita (World Scientific, Singapore 1990) p. 419.

[92] V. Hughes, in Frontiers of High Energy Spin Physics, ed. by T. Hasegawa et al. (Universal Academy Press, Tokyo 1992), p. 717.

[93] T. Kinoshita, B. Nizic, and Y. Okamoto, Phys. Rev. D41, 593 (1990); T. Kinoshita, Phys. Rev. D47, 5013 (1993); M. Samuel and G. Li, Phys. Rev. D44, 3955 (1991,)Phys. Rev. D48, 1879(E) (1993); S. Laporta Phys. Rev. D47, 4793 (1993); S. Karshenboim, Yad. Fiz. 56, 252 (1993) [Phys. At. Nucl. 58857 (1993)].

[94] S. Eidelman and F. Jegerlehner, PSI-PR-95-1, hep-ph/9502298.

[95] M. Hayakawa, T. Kinoshita, and A. Sanda, Phys. Rev. Lett. 75, 790 (1995); J. Bijnens, E. Pallante, and J. Prades, Phys. Rev. Lett. 75, 1447 (1995).

[96] T. Kukhto, E. Kuraev, A. Schiller, and Z. Silagadze, Nucl. Phys. B371, 567 (1992); A. Czarnecki, B. Krause, and W. Marciano, Phys. Rev. D52, R2619 (1995).

[97] P. Méry, S. Moubarik, M. Perrottet, and F. Renard, Z. Phys. C46, 229 (1990); C. Arzt, M. Einhorn, and J. Wudka, Phys. Rev. D49, 1370 (1994).

[98] J. Grifols and A. Mendez, Phys. Rev. D26, 1809 (1982); J. Ellis, J. Hagelin, and D. Nanopoulos, Phys. Lett. 116B, 283 (1982); R. Barbieri and L. Maiani, Phys. Lett. 117B, 203 (1982); D. Kosower, L. Krauss, and N. Sakai, Phys. Lett. 133B, 305 (1983); T. C. Yuan, R. Arnowitt, A. Chamseddine, and P. Nath, Z. Phys. C26, 407 (1984); J. Lopez, D. Nanopoulos, X. Wang, Phys. Rev. D49, 366 (1994). 
[99] U. Chattopadhyay and P. Nath, NSF-ITP-95-64, hep-ph/9507386.

[100] G. Beall, M. Bander, and A. Soni, Phys. Rev. Lett. 48, 848 (1992).

[101] See, for example, J. Ellis and D.V. Nanopoulos, Phys. Lett. 110B, 44 (1982).

[102] S. Dimopoulos and J. Ellis, Nucl. Phys. B182, 505 (1981); S. Dimopoulos, H. Georgi, and S. Raby, Phys. Lett. 127B, 101 (1983); R.S. Chivukula and H. Georgi, Phys. Lett. B188, 99 (1987); R.S. Chivukula, H. Georgi, and L. Randall, Nucl. Phys. B292, 93 (1987); L. Randall Nucl. Phys. b403, 122 (1993).

[103] For recent reviews and original references, see L. Littenberg and G. Valencia, Ann. Rev. Nucl. Part. Sci. 43, 729 (1993); B. Winstein, L. Wolfenstein, Rev. Mod. Phys. 65, 1113 (1993); J.L. Ritchie, S.G. Wojcicki, Rev. Mod. Phys. 65, 1149 (1993); R. Battiston, et al., Phys. Rep. 214293 (1992); A. Buras, M. Jamin, and M. Lautenbacher, Nucl. Phys. B408, 209 (1993).

[104] Y. Kuno, Nucl. Phys. (Proc. Suppl.) 37A, 87 (1994); Chin. J. of Phys. 32, 6 (1994).

[105] E. Gabrielli, A. Masiero, and L. Siilvestrini (1995) hep-ph/9509379 and hep/ph/9510215; J. Hagelin, S. Kelley, and T. Tanaka, Nucl. Phys. B415, 293 (1994), and Mod. Phys. Lett. A8, 2737 (1993).

[106] B. Winstein and L. Wolfenstein in [103].

[107] P. Langacker, S. Sankar, and K. Schilcher, Phys. Rev. D38, 2841 (1988).

[108] L. Littenberg and G. Valencia, in [103.

[109] J.L. Ritchie and S.G. Wojcicki in [103].

[110] C.Q. Geng, I.J. Hsu, Y.C. Lin, Phys. Lett. B355, 569 (1995); S. Fajfer, Helsinki University Report HU-SEFT-R-1996-05, hep-ph/9602322.

[111] G. Buchalla and A. Buras, Nucl. Phys. B412, 106 (1994).

[112] I. Bigi and F. Gabbiani, Nucl. Phys. B367, 3 (1991).

[113] J.S. Hagelin, L.S. Littenberg, Prog. Part. Nucl. Phys. 23, 1 (1989).

[114] C.Q. Geng and J.N. Ng, Phys. Rev. D42, 1509 (1990).

[115] C. Dib, I. Dunietz, and F.J. Gilman, Phys. Lett. B218, 487 (1989).

[116] G. Bélanger, C.Q. Geng, Phys. Rev. D44, 2789 (1991). 
[117] S. Dawson and G. Valencia, Phys. Rev. D49, 2188 (1994).

[118] T. Inami and C.S. Lim, Prog. Theor. Phys. 65, 297 (1981).

[119] A. Fryberger et al., CLEO Collaboration, CLNS Report 96/1389 (1996); M. Selen, CLEO Collaboration, talk presented at APS Spring Meeting, Washington D.C., April 1994; T. Alexopoulos et al., E771 Collaboration, Fermilab Report Fermilab-Pub-95-286-E (1995).

[120] G. Burdman, E. Golowich, J.L. Hewett, and S. Pakvasa, Phys. Rev. D52, 6383 (1995).

[121] T.E. Browder and S. Pakvasa, Univ. of Hawaii Report UH-511-828-95 (1995); L. Wolfenstein, Carnegie Mellon Univ. Report CMU-HEP-95-04 (1995); G. Blaylock, A. Seiden, and Y. Nir, Univ. of Santa Cruz Report SCIPP-95-16 (1995).

[122] A. Datta, Z. Phys. C27, 515 (1985).

[123] G. Burdman, E. Golowich, J.L. Hewett, and S. Pakvasa, SLAC Report SLACPUB-7136.

[124] H. Georgi, Phys. Lett. B297, 353 (1992); T. Ohl et al., Nucl. Phys. B403, 605 (1993).

[125] K.S. Babu et al., Phys. Lett. B205, 540 (1988); T.G. Rizzo, Int. J. Mod. Phys. A4, 5401 (1989).

[126] M.S. Alam et al., CLEO Collaboration, Phys. Rev. Lett. 74, 2885 (1995); R. Ammar et al., CLEO Collaboration, Phys. Rev. Lett. 71, 674 (1993); K.W. Edwards et al., CLEO Collaboration, presented at 1995 Meeting of the European Physical Society Conference on High Energy Physics, Brussels, Belgium, July 1995, CLEO-CONF95-6.

[127] Y. Nir and N. Seiberg, Phys. Lett. B309, 337 (1993).

[128] F. Bucella et al., Phys. Lett. B302, 319 (1993); G. Burdman in Proceedings of the CHARM2000 Workshop, Fermilab 1994, ed. D. Kaplan.

[129] P.L. Frabetti et al., Phys. Rev. D50, 2953 (1994); J. Bartelt et al., CLEO Collaboration, Phys. Rev. D52, 4860 (1995).

[130] A. Le Yaouanc et al., Phys. Lett. B292, 353 (1992); S. Pakvasa, in Proceedings of CHARM2000 Workshop, Fermilab, June 1994, ed. D. Kaplan; M. Gronau and S. Wakaizumi, Phys. Rev. Lett. 68, 1814 (1992).

[131] I.I. Bigi, F. Gabbiani, and A. Masiero, Z. Phys. C48, 633 (1990). 
[132] For a review of present experiment results on rare $B$ physics, see, T.E. Browder, K. Honscheid, and S. Playfer, in $B$ Decays, $2^{\text {nd }}$ edition, ed. S. Stone, (World Scientific 1995).

[133] D. Boutigny et al., BABAR Collaboration, SLAC Report SLAC-0443 (1994); M.T. Cheng et al., BELLE Collaboration, KEK Report KEK-94-02 (1994); The CLEO III Detector Proposal, Cornell Report CLNS-94/1277 (1994); The CDF Collaboration, CDF-DOC-ADVISORY-PUBLIC/2436; T. Lohse et al., HERA-B Collaboration, DESY Report DESY-PRC-93-04 (1994); K. Kirsebom et al., LHC-B Collaboration, CERN Report CERN/LHCC 95-5 (1995).

[134] M. Artuso et al., CLEO Collaboration, Phys. Rev. Lett. 75, 785 (1995); D. Buskulic et al., ALEPH Collaboration, Phys. Lett. B343, 444 (1995).

[135] For a review of implications of non-SM physics in $b \rightarrow s \gamma$, see, J.L. Hewett, in 21st Annual SLAC Summer Institute on Particle Physics, Stanford, Ca, July 1993, hep-ph/9406302.

[136] A.J. Buras et al., Nucl. Phys. B424, 374 (1994), and references therein.

[137] T. Skwarnicki, talk presented at XVII International Conference on LeptonPhoton Interactions, Beijing, China, July 1995, hep-ph/9512395.

[138] D. Atwood, B. Blok, and A. Soni, SLAC Report SLAC-PUB-95-6635 (1995); N.G. Deshpande, X.-G. He, and J. Trampetic, Phys. Lett. 367, 362 (1996); H.Y. Cheng, Report IP-ASTP-23-94; J. Milana, Phys. Rev. D53, 1403 (1996); G. Ricciardi, Phys. Lett. B358, 129 (1995); A. Ali and V.M. Braun, Phys. Lett. B359, 223 (1995).

[139] J.L. Hewett, Phys. Lett. B193, 327 (1987); W.-S. Hou, A. Soni, and H. Steger, Phys. Lett. B192, 441 (1987).

[140] T.G. Rizzo, Phys. Rev. D38, 820 (1988); X.G. He, T.D. Nguyen, and R.R. Volkas, Phys. Rev. D38, 814 (1988); W.-S. Hou and R.S. Willey, Phys. Lett. B202, 591 (1988); C.Q. Geng and J.N. Ng, Phys. Rev. D38, 2858 (1988); B. Grinstein, R. Springer, and M. Wise, Nucl. Phys. B339, 269 (1990).

[141] J.L. Hewett, Phys. Rev. Lett. 70, 1045 (1993); V. Barger, M. Berger, and R.J.N. Phillips, Phys. Rev. Lett. 70, 1368 (1993).

[142] S. Bertolini, et al., Nucl. Phys. B294, 321 (1987), and Nucl. Phys. B353, 591 (1991); R. Barbieri and G.F. Giudice, Phys. Lett. B309, 86 (1993); R. Garisto and J.N. Ng, Phys. Lett. B315, 119 (1993); M.A. Diaz, Phys. Lett. B322, 207 (1994); F.M. Borzumati Z. Phys. C63, 291 (1994); S. Bertolini and F. Vissani, Z. Phys. C67, 513 (1995); J.L. Lopez et al., Phys. Rev. D48, 974 (1993); H. Anlauf, Nucl. Phys. B430, 245 (1994). 
[143] T. Goto and Y. Okada, Prog. Theor. Phys. 94, 407 (1995).

[144] G.L. Kane, C. Kolda, L. Roszkowski, and J.D. Wells, Phys. Rev. D49, 6173 (1994).

[145] F.M. Borzumati, M. Drees, and M. Nojiri, Phys. Rev. D51, 341 (1995); P. Nath and R. Arnowitt, Phys. Rev. Lett. 74, 4592 (1995), and Phys. Lett. B336, 395 (1994).

[146] T.G. Rizzo, Phys. Lett. B315, 471 (1993); S.-P. Chia, Phys. Lett. B240, 465 (1990); K.A. Peterson, Phys. Lett. B282, 207 (1992); U. Baur, in Proceedings of the Workshop on B Physics at Hadron Accelerators, Snowmass, CO, June 1993, ed. P. McBride and C. Shekhar Mishra, SSCL-SR-1225, FermilabCONF-93/267.

[147] J. Ellison, D0 Collaboration, Proceeding of 1994 Meeting of the Division of Particles and Fields, Albuquerque, NM (1994). For comparable bounds from CDF and UA2, see F. Abe et al., CDF Collaboration Phys. Rev. Lett. 74, 1936 (1995); J. Alitti et al., UA2 Collaboration, Phys. Lett. B277, 194 (1992).

[148] J.L. Hewett and T.G. Rizzo, Phys. Rev. D49, 319 (1994).

[149] For estimates on the exclusive decay rates, see A. Ali, C. Greub, and T. Mannel, in Proceedings of the ECFA Workshop on a European B Meson Factory, Hamburg Germany, DESY-93-016. We thank A. Ali and C. Greub for informing us of recent updates on these numbers. For estimates on $B \rightarrow \gamma \gamma$, see S. Herrlich and J. Kalinowski, Nucl. Phys. B381, 501 (1992).

[150] F. Abe et al., CDF Collaboration, Fermilab-PUB-96/1040-E; R. Ammar et al., CLEO Collaboration, Phys. Rev. D49, 5701 (1994); R. Balest et al., CLEO Collaboration, in the Proceedings of the $27^{\text {th }}$ International Conference on High Energy Physics, Glasgow, Scotland, July 1994, ed. P.J. Bussey and I.G. Knowles (Institute of Physics, Bristol 1995).

[151] Y. Grossman, Z. Ligeti, and E. Nardi, Weizmann Institute Report WIS95/49, hep-ph/9510378,

[152] L. Randall and R. Sundrum, Phys. Lett. B312, 148 (1993).

[153] J.L. Hewett, S. Nandi, T.G. Rizzo, Phys. Rev. D39, 250 (1989); M. Savage, Phys. Lett. B266, 135 (1991).

[154] B. Grinstein, Y. Nir, and J.M. Soares, Phys. Rev. D48, 3960 (1993).

[155] A. Ali, G.F. Giudice, and T. Mannel, Z. Phys. C67, 417 (1995); A. Ali, T. Mannel, and T. Morozumi, Phys. Lett. B273, 505 (1991). 
[156] A.J. Buras and M. Münz, Phys. Rev. D52, 186 (1995).

[157] N.G. Deshpande and J. Trampetic, Phys. Rev. Lett. 60, 2583 (1988); C.S. Lim, T. Morozumi, and A.I. Sanda, Phys. Lett. B218, 343 (1989); N.G. Deshpande, J. Trampetic, and K. Panrose, Phys. Rev. D39, 1461 (1989); B. Grinstein, M.J. Savage, and M.B. Wise, Nucl. Phys. B319, 271 (1989).

[158] J.L. Hewett, SLAC Report, SLAC-PUB-95-6820, hep-ph/9506289 (Phys. Rev. D, in press).

[159] A.J. Buras, M. Jamin, P.H. Weisz, Nucl. Phys. B347, 491 (1990).

[160] A. Soni, talk presented at the 1995 International Symposium on Lattice Gauge Theory, Melbourne, Australia, July 1995, hep-lat/9510036.

[161] S.-L. Wu, talk presented at XVII International Symposium on Lepton-Photon Interactions, Beijing, China, August 1995, hep-ex/9602003.

[162] T. Goto, T. Nihei, and Y. Okada, KEK Report KEK-TH-445, hepph/9510286; G. Couture and H. König, Z. Phys. C69, 499 (1996), and hepph/9511234; G.C. Cho, Y. Kizukuri, and N. Oshimo, hep-ph/9509277.

[163] M. Kobayashi and T. Maskawa, Prog. Theor. Phys. 49, 652 (1973).

[164] L. Wolfenstein, Phys. Rev. Lett. 51, 1945 (1983).

[165] Y. Grossman and Y. Nir, Phys. Lett. B313, 126 (1993).

[166] G.C. Branco, G.C. Cho, Y.Kizukuri, and N. Oshimo, Phys. Lett. B337, 316 (1994), and Nucl. Phys. B449, 483 (1995).

[167] For a recent review, see, Y. Nir, in Workshop on B Physics at Hadron Accelerators, Snowmass, CO, June 1993, ed. P. McBride and C. Shekhar Mishra, SSCL-SR-1225, Fermilab-CONF-93/267.

[168] D. Silverman, hep-ph/9504387.

[169] G. Eilam, J.L. Hewett, and A. Soni, Phys. Rev. D44, 1473 (1991).

[170] B. Grzadkowski, J.F. Gunion, and P. Krawczyk, Phys. Lett. B286, 106 (1991); N.G. Deshpande, B. Margolis, and H. Trottier, Phys. Rev. D45, 178 (1992); M. Luke and M. Savage, Phys. Lett. B307, 387 (1993).

[171] C.S. Li, R.J. Oakes, and J.M. Yang, Phys. Rev. D49, 293 (1994); G. Couture, C. Hamzaoui, and H. Konig, Phys. Rev. D52, 1713 (1995). 
[172] T. Han, R.D. Peccei, and X. Zhang, Nucl. Phys. B454, 527 (1995); T. Han et al., Univ. California Davis Report UCD-96-07, hep-ph/9603247; E. Malkawi and T. Tait, hep-ph/9511337

[173] T.J. LeCompte, CDF Collaboration, Presented at 2nd Rencontres du Vietnam, Ho Chi Minh City, Vietnam, October 1995, Fermilab-CONF-96/021-E.

[174] I.I. Bigi et al., Phys. Lett. 181B, 157 (1986); V. Barger and R.J.N. Phillips, Phys. Rev. D41, 884 (1990), ibid.. D40, 2875 (1990), and Phys. Lett. B201, 553 (1988); C.S. Li and T.C. Yuan, Phys. Rev. D42, 3088 (1990).

[175] T.P. Cheng and M. Sher, Phys. Rev. D35, 3483 (1987); B. Mukhopadhyaya and S. Nandi, Phys. Rev. Lett. 66, 285 (1991); T.P. Cheng and L.F. Li, Phys. Rev. D45, 1708 (1992); W.-S. Hou, Phys. Lett. B296, 179 (1992); L.J. Hall and S. weinberg, Phys. Rev. D48, R979 (1993); W.-S. Hou and H.C. Huang, Phys. Rev. D51, 5285 (1995).

[176] K. Hikasa and M. Kobayashi, Phys. Rev. D36, 724 (1987); H. Baer et al., Phys. Rev. D44, 725 (1991); J. Sender hep-ph/9602354.

[177] K. Agashe and M. Graesser, LBNL Report LBNL-37823 (1995), hepph/9510439.

[178] See, for example, G. Eilam, J.L. Hewett, and A. Soni, Phys. Rev. Lett. 67, 1979 (1991), ibid., 68, 2103 (1992).

[179] J.F. Donoghue and G. Valencia, Phys. Rev. Lett. 58, 451 (1987), and E60, 243 (1988); C.A. Nelson, Phys. Rev. D43, 1465 (1991); C.R. Schmidt and M.E. Peskin, Phys. Rev. Lett. 69, 410 (1992); W. Bernreuther et al., Nucl. Phys. B388, 53 (1992), and EB406, 516 (1993); A. Brandenburg and J.P. Ma, Phys. Lett. B298, 211 (1993), and Z. Phys. C56, 97 (1992); B. Grzadkowski, Phys. Lett. B305, 384 (1993); B. Grzadkowski and W.-Y. Keung, Phys. Lett. B319, 526 (1993), and ibid., B316, 137 (1993); D. Atwood et al., Phys. Rev. Lett. 70, 1364 (1993); T. Arens and L.M. Sehgal, Nucl. Phys. B393, 46 (1993), Phys. Lett. B302, 501 (1993), and Phys. Rev. D50, 4372 (1994); W. Bernreuther and A. Brandenburg, Phys. Lett. B314, 104 (1993), and Phys. Rev. D49, 4481 (1994); D. Chang, W.-Y. Keung, I. Phillips, Nucl. Phys. B408, 286 (1993), and EB429, 255 (1994); W. Bernreuther and P. Overmann, PITHA 95/30 (1995).

[180] S. Murthy, D. Krause, Z. Li, and L. Hunter, Phys. Rev. Lett. 63, 965 (1989).

[181] K. Abdullah, C. Carlberg, E. Commins, H. Gould, and S. Ross, Phys. Rev. Lett. 65, 2347 (1990); E. Commins, S. Ross, D. Demille, and C. Regan, Phys. Rev. A50, 2960 (1994). 
[182] T. Vold, F. Raab, B. Heckel, and E. N. Fortson, Phys. Rev. Lett. 52, 2229 (1984); E. Oteiza, R. Hoare, T. Chupp, Bull. Am. Phys. Soc. 37, 947 (1992).

[183] J. Jacobs, W. Klipstein, S. Lamoreaux, B. Heckel, and E. N. Fortson, Phys. Rev. Lett. 71, 3782 (1993); Phys. Rev. A52, 3521 (1995).

[184] D. Cho, K. Stangster, and E. Hinds, Phys. Rev. Lett. 63, 2559 (1989); Phys. Rev. A44, 2783 (1991).

[185] K. Smith et al., Phys. Lett. B234, 191 (1990); I. Altarev et al., Phys. Lett. B276, 242 (1992).

[186] J. Ellis, S. Ferrara, and D. Nanopoulos, Phys. Lett. B114 (1982); J. Polchinski and M. Wise, Phys. Lett. B125, 393 (1983).

[187] W. Fischler, S. Paban, and S. Thomas, Phys. Lett. B289, 373 (1992).

[188] W. Bernreuther and M. Suzuki, Rev. Mod. Phys. 63, 313 (1991); S. Barr, Int. J. Mod. Phys. A8, 209 (1993).

[189] S. Dimopoulos and L. Hall, Phys. Lett. B344, 185 (1995).

[190] R. Barbieri, A. Romanino, and A. Strumia, IFUP-TH-65-95; hep$\mathrm{ph} / 9511305$.

[191] S. Bertolini and F. Vissani, Phys. Lett. B324, 164 (1994); T. Inui, Y. Mimura, N. Sakai, and T. Sasaki, Nucl. Phys. B449, 49 (1995).

[192] Y. Kizukuri and N. Oshimo, Phys. Rev. D45, 1806 (1992); Phys. Rev. D46, 3025 (1992).

[193] J. Feng, N. Polonsky, and S. Thomas, SLAC-PUB-95-7050, hep-ph/9511324, to appear in Phys. Lett. B.

[194] S. Dimopoulos and S. Thomas, SLAC-PUB-95-7010, hep-ph/9510220, to appear in Nucl. Phys. B.

[195] S. Barr and A. Zee, Phys. Rev. Lett. 65, 21 (1990); J. Gunion and R. Vega, Phys. Lett. B251, 157 (1990); D. Chang, W. Keung, and T. Yuan, Phys. Rev. D43, R14 (1991); R. Leigh, S. Paban, and R. Xu, Nucl. Phys. B352, 45 (1991).

[196] J. Gunion and D. Wyler, Phys. Lett. B248, 170 (1990); D. Chang, W. Keung, and T. Yuan, Phys. Lett. B251, 608 (1990). 
[197] T.S. Komas, G.K. Leontaris, and J.D. Vergados, Prog. Part. Nucl. Phys. 33, 397 (1994); G. Mann and T. Riemann, Annalen Phys. (Leipzig) 40, 334 (1983); M.J.S. Levine, Phys. Rev. D36, 1329 (1987); A. Mendez and L.M. Mir, Phys. Rev. D40, 251 (1989); T.K. Kuo and N. Nakagawa, Phys. Rev. D32, 306 (1985); J. Bernabeu et al., Phys. Lett. B187, 303 (1987); J. Bernabeu and A. Santamaria, Phys. Lett. B197, 418 (1987); A, Iiakovac and A. Pilaftsis, Nucl. Phys. B437, 491 (1995); M. Dittmar and J.W.F. Valle, in CERN Yellow Report, CERN-91-02, March 1991; G. Eilam and T.G. Rizzo, Phys. Lett. B188, 91 (1987).

[198] R. Bolton, et al., Phys. Rev. D38, 2077 (1988).

[199] U. Bellgardt, et al., SINDRUM Collaboration, Nucl. Phys. B299, 1 (1988).

[200] C. Dohman, et al., SINDRUM Collaboration, Phys. Lett. B317, 631 (1993).

[201] A. Bean, et al. CLEO Collaboration, Phys. Rev. Lett. 70, 138 (1993).

[202] The OPAL Collaboration, CERN-PPE/95-43 (1995).

[203] The L3 Collaboration, submitted to the 1995 EPS and Lepton Photon Conferences, July 1995 L3-Note-1798.

[204] M. Cooper (Spokesman) LAMPF proposal No. 969 (1985).

[205] A. Van der Schaff (Spokesman) PSI proposal R-87-03 (1987).

[206] R. Barbieri and L. Hall, Nucl. Phys. B338, 212 (1994); R. Barbieri, L. Hall, and A. Strumia, Nucl. Phys. B445, 219 (1995).

[207] A. Balysh et al., Phys. Lett. B356 , 450 (1995).

[208] R. N. Mohapatra and G. Senjanović, Phys. Rev. Lett. 44, 912 (1980); Phys. Rev. D23, 165 (1981).

[209] P. Langacker and S.U. Sankar, Phys. Rev. D40, 1569 (1989).

[210] M. Doi, T. Kotani, E. Takasugi, Prog. Theor. Phys. Suppl. 83, 1 (1985).

[211] H. Klapdor-Kleingrothaus, Prog. in Part. Nucl. Phys., B32, 261 (1994); A. Balysh et al., Phys. Lett. ( to appear).

[212] K. S. Babu and R. N. Mohapatra, Phys. Rev. Lett. 75, 2276 (1995).

[213] R.N. Mohapatra, Phys. Rev. D34, 909 (1986).

[214] M. Schwarz, Phys. Rev. D40, 1521 (1989). 
[215] R. N. Mohapatra, Phys. Rev. D34, 3457 (1986).

[216] M. Hirsch, H. Klapdor-Kleingrothaus and S. Kovalenko, Heidelberg preprint (1995).

[217] O. Panella and Y. N. Srivastava, College de France Preprint, LPC 94-39; E. Takasugi, hep-ph/9506379.

[218] P. Bamert, C. Burgess and R. N. Mohapatra, Nucl. Phys. B438, 3 (1995). 